\title{
APPROXIMATION OF $G$-MAPS BY MAPS IN EQUIVARIANT GENERAL POSITION AND IMBEDDINGS OF $G$-COMPLEXES
}

\author{
BY
}

\author{
SÖREN ILLMAN
}

\begin{abstract}
Let $G$ be a finite group. In this paper we consider maps $f: P \rightarrow M$ from equivariant polyhedra into equivariant p.l. manifolds. We prove an equivariant general position result which shows how to approximate a given continuous proper equivariant (or isovariant) map $f: P \rightarrow M$ by a $G$-map which is in equivariant general position. We also apply this equivariant general position result to get a general $G$-imbedding theorem. Applied to the case of $G$-imbeddings of simplicial $G$-complexes into euclidean representation space this general $G$-imbedding theorem gives a result which provides a good hold on the required dimension of the euclidean representation space. For example in the case when $G=Z_{m}$ we prove that there exists a representation space $R^{r(k, m)}(\rho)$ with the property that any $k$-dimensional simplicial $Z_{m}$-complex $X$ admits a proper p.l. $Z_{m}$-imbedding into $\mathbf{R}^{r(k, m)}(\rho)$ and we also show that the dimension $r(k, m)$ is best possible, i.e., one cannot find a euclidean representation space of lower dimension than $r(k, m)$ with the same property as $\mathbf{R}^{r(k, m)}(\rho)$. Simple explicit expressions for the dimension $r(k, m)$ are given. We also consider the case of semi-free actions with a given imbedding of the fixed point set into some euclidean space. Furthermore we show that the p.l. $G$-imbeddings of equivariant p.l. manifolds into euclidean representation space obtained by our $G$-imbedding results are in general equivariantly locally knotted although they are locally flat in the ordinary sense. This phenomenon can occur in arbitrarily high codimensions.
\end{abstract}

Introduction. Let $G$ be a finite group. In this paper we prove an equivariant general position result which shows how to approximate a given continuous proper equivariant (or isovariant) map, from an equivariant simplicial complex into an equivariant p.l. manifold, by a $G$-map in equivariant general position, see Theorem 3.10 and the Definition preceding Proposition 3.9. (All simplicial complexes are assumed to be countable, locally finite and finite-dimensional. For the notion of an equivariant p.l. manifold, see [8] or $\S 1$ of this paper.) Our original motivation for proving an equivariant general position result was in applications to equivariant engulfing and other similar techniques in equivariant p.l. topology. In this paper we apply our equivariant general position theorem to obtain $G$-imbedding results. We prove the following general $G$-imbedding theorem; see Theorem 3.11.

Theorem A. Let $P$ be an equivariant polyhedron, $Q$ an equivariant subpolyhedron of $P$ and $M$ an equivariant p.l. manifold, and let $\varepsilon: P \rightarrow \mathbf{R}$ be any given invariant

Received by the editors February 6, 1979.

AMS (MOS) subject classifications (1970). Primary 57E99, 57C35; Secondary 57C55.

Key words and phrases. Equivariant general position, equivariant imbeddings. 
positive function. Let $f: P \rightarrow M$ be a proper $G$-map such that $f \mid Q$ is a p.l. G-imbedding. Let $\left(H_{1}\right), \ldots,\left(H_{v}\right)$ be the orbit types occurring in $P-Q$, and assume that

(i) $\operatorname{dim} P_{\alpha}^{H_{i}} \leqslant \operatorname{dim} M_{f(\alpha)}^{H_{i}}-\operatorname{dim} M_{f(\alpha)}^{>H_{i}}-1$,

(ii) $2 \operatorname{dim} P_{\alpha}^{H_{i}} \leqslant \operatorname{dim} M_{f(\alpha)}^{H_{i}}-1$

for $i=1, \ldots, v$ and any component $P_{\alpha}^{H_{i}}$ of $P^{H_{i}}$. Then $f$ is equivariantly $\varepsilon$-homotopic rel $|Q|$ to a proper p.l. G-imbedding $h: P \rightarrow M$.

Here $M_{f(\alpha)}^{H}$ denotes the component of $M^{H}$ which contains $f\left(P_{\alpha}^{H}\right)$, and $M_{f(\alpha)}^{>H}$ is the set of points in $M_{f(\alpha)}^{H}$ with isotropy subgroup strictly greater than $H$. If we moreover assume that $f$ is isovariant then condition (ii) alone is sufficient for the existence of a $G$-imbedding that approximates $f$ arbitrarily well; see Theorem 3.12. We feel that these two $G$-imbedding results form the appropriate generalization of the classical result, in the ordinary nonequivariant case, on the existence of imbeddings of a polyhedron $P$ into a $(2 \operatorname{dim} P+1)$-dimensional p.l. manifold $M$.

In $\$ 4$ we apply our general $G$-imbedding result, Theorem $A$, to the case of $G$-imbeddings into euclidean representation space. By the theorem of Mostow [10], every action of a compact Lie group with a finite number of orbit types on a finite-dimensional separable metrizable space can be equivariantly imbedded into a euclidean representation space for the group. But Mostow's theorem does not say anything about the required dimension of the euclidean representation space. Copeland and de Groot showed in [3] that every action of a cyclic group of prime order on a $k$-dimensional separable metrizable space can be equivariantly imbedded into a euclidean representation space of dimension $3 k+2$ or $3 k+3$, and they also proved that this result is best possible. In [9] Kister and Mann obtained results on the required dimension of the euclidean representation space in the case of actions of compact abelian Lie groups with a finite number of distinct isotropy subgroups, on finite-dimensional separable metrizable spaces. For $G$ an arbitrary finite group our result (see Theorem 4.2), is

TheOREM B. Let $X$ be a simplicial $G$-complex and $X_{0}$ a G-subcomplex of $X$, and let $\left(H_{1}\right), \ldots,\left(H_{v}\right)$ be the orbit types occurring in $X-X_{0}$. Let $\mathbf{R}^{r}(\rho)$ be a representation space for $G$ such that there exists a proper p.l. G-imbedding $h_{0}: X_{0} \rightarrow \mathbf{R}^{r}(\rho)$ and assume that

(i) $\operatorname{dim} X^{H_{i}} \leqslant \operatorname{dim}\left[\mathbf{R}^{r}(\rho)\right]^{H_{i}}-\operatorname{dim}\left[\mathbf{R}^{r}(\rho)\right]^{>H_{i}}-1$,

(ii) $2 \operatorname{dim} X^{H_{i}} \leqslant \operatorname{dim}\left[\mathbf{R}^{r}(\rho)\right]^{H_{i}}-1$

for $i=1, \ldots, v$. Then $h_{0}$ can be extended to a proper p.l. G-imbedding $h: X \rightarrow$ $\mathbf{R}^{r}(\rho)$.

The case $X_{0}=\varnothing$ gives the wanted absolute version. When Theorem B is applied to the case of an arbitrary finite cyclic group we get the following; see Corollary 4.3.

Corollary C. Let $G=Z_{m}$, where $m=p_{1}^{a_{1}} \ldots p_{n}^{a_{n}}$ and $p_{1}, \ldots, p_{n}$ are distinct primes. Then there exists a euclidean representation space $\mathbf{R}^{r(k, m)}(\rho)$, where 


$$
r(k, m)= \begin{cases}2 k+1+\left(a_{1}+\ldots+a_{n}\right)(k+2) & \text { if } k \text { is even and } m \text { is odd, } \\ 2 k+\left(a_{1}+\ldots+a_{n}\right)(k+2) & \text { if } k \text { is even and } m \text { is even, } \\ 2 k+1+\left(a_{1}+\ldots+a_{n}\right)(k+1) & \text { if } k \text { is odd, }\end{cases}
$$

such that any $k$-dimensional simplicial $Z_{m}$-complex $X$ has a proper p.l. $Z_{m}$-imbedding into $\mathbf{R}^{r(k, m)}(\rho)$.

We prove in Proposition 4.4 that this result is best possible. In case $m=p$, a prime, the dimension given by Corollary $\mathbf{C}$ is the same as the one in the Copelandde Groot result. In the case of actions by cyclic groups of prime power order, the Kister-Mann result (see [9, Theorem 2]) gives almost the same dimension as in Corollary $\mathrm{C}$; but for example in the case of $Z_{m}$-actions, where $m=p_{1} \ldots p_{n}$ and $p_{1}, \ldots, p_{n}$ are distinct primes, on $k$-dimensional space, $k$ odd, the Kister-Mann result gives the dimension $\left(2^{n}+1\right) k+2^{n}$ whereas the best possible dimension, given by Corollary $\mathrm{C}$, is $(n+2) k+n+1$. In the case of actions by an arbitrary finite abelian group, we also obtain a corresponding improvement, compared with the Kister-Mann result, of the required dimension of the euclidean representation space, see Corollary 4.7.

If one considers only semifree actions and assumes that the fixed point set is imbeddable in euclidean space $\mathbf{R}^{d}$, one gets very low required dimensions for the euclidean representation space. In the case of compact, finite-dimensional metric $Z_{m}$-spaces such a result has been proved by Allen (see [1, Theorems 1.1 and 1.2]). Our corresponding result (Corollary 4.5 ) in the case of simplicial $Z_{m}$-complexes obtains the same dimensions as in the result by Allen. We also give a result of the same type in the case of actions by an arbitrary finite abelian group; see Corollary 4.6.

In order to give a specific numerical application of Theorem B also in the case of a nonabelian group, we show that for $G=S_{3}$, the symmetric group on three letters, there exists a euclidean representation space $\mathbf{R}^{5 k+4}(\rho)$ of dimension $5 k+4$ such that every $k$-dimensional simplicial $S_{3}$-complex has a proper p.l. $S_{3}$-imbedding into $\mathbf{R}^{5 k+4}(\rho)$.

Furthermore we show in $\$ 4$ that the p.l. $G$-imbeddings of equivariant p.l. manifolds into euclidean representation space given by Theorem $\mathbf{B}$ are in general equivariantly locally knotted although they are locally flat in the ordinary sense. This phenomenon can occur in arbitrarily high codimensions. Among other things this shows that the existence of p.l. and smooth $G$-imbeddings are questions of completely different natures. (Recall in this connection that every smooth $G$-manifold has a uniquely determined structure of an equivariant p.l. manifold, see [8].) For results concerning the existence of smooth $G$-imbeddings we refer to Wasser$\operatorname{man}[15]$.

Let us now describe the outline for the proof of our equivariant general position result, Theorem 3.10. The proof falls into three main steps, each of which has a local and global part. The local part deals with $G$-maps from a finite equivariant simplicial complex $X$ into some orthogonal representation space $\mathbf{R}^{m}(\rho)$, and with equivariant general position of compact equivariant subpolyhedra in $D^{m}(\rho)$. The 
local results are proved in $\$ 2$. The global part deals with $G$-maps from equivariant polyhedra into equivariant p.l. manifolds. In all three steps the globalization consists of a patching together process of the corresponding local result in order to get the global result. The globalization is carried out in \$3. All the results are established in relative forms. Of the three main steps the first is to approximate the given equivariant or isovariant map $f$ by an equivariant or isovariant, respectively, p.l. map $f_{1}$. In the case when $f$ is equivariant this is standard. The case when $f$ is isovariant is new and of independent interest, and it requires considerably more care than the case when $f$ is equivariant. See Lemma 2.3, Theorem 3.3 and Corollary 3.4. In this connection we should point out that M. H. Schwartz in [14] proves that stratified continuous maps have stratified simplicial approximations (there is an extra assumption in the relative case). Next the equivariant or isovariant p.l. map $f_{1}$ is approximated by an equivariantly nondegenerate p.l. map $f_{2}$. In particular $f_{2}$ is always isovariant. This second step requires some dimension assumptions. See Lemma 2.5 and Theorem 3.5, and Lemma 2.8 and Theorem 3.7. As a by-product we obtain the following result on approximation of an equivariant map by an isovariant map, which is of independent interest. (See the Remark after Theorem 3.7.)

THEOREM. Let $f: P \rightarrow M$ be a proper $G$-map. Let $\left(H_{1}\right), \ldots,\left(H_{v}\right)$ be the orbit types occurring in $P$ and assume that

$$
\operatorname{dim} P_{\alpha}^{H_{i}} \leqslant \operatorname{dim} M_{f(\alpha)}^{H_{i}}-\operatorname{dim} M_{f(\alpha)}^{>H_{i}}-1
$$

for $i=1, \ldots, v$ and each component $P_{\alpha}^{H_{i}}$ of $P^{H_{i}}$. Then $f$ is equivariantly $\varepsilon$-homotopic to an isovariant proper (and p.l.) map $h: P \rightarrow M$.

For the third step we first prove that one can, by an equivariant ambient isotopy, move any equivariant subpolyhedron of $M$ into equivariant general position with respect to any finite number of equivariant subpolyhedra of $M$, see Lemma 2.12 and Proposition 3.8. Using this result we show that the equivariantly nondegenerate p.l. map $f_{2}$ can be approximated by a map $f_{3}$ which is in equivariant general position. See Proposition 3.9 for a precise statement. All three steps together gives us our equivariant general position result Theorem 3.10. The general $G$-imbedding result Theorem 3.11 and its isovariant version Theorem 3.12 now follow easily.

The first start on this work was made in the summer of 1975 at the Institute for Advanced Study in Princeton, but the work was then put aside. At the Conference on Transformation Groups in Newcastle upon Tyne in August 1976, the author spoke about part of it and the results concerning approximation of equivariant or isovariant p.l. maps by equivariantly nondegenerate p.l. maps (in the case of maps from finite equivariant simplicial complexes) were announced in [7]. Most of this work was completed while the author was on leave from the University of Helsinki and enjoyed the hospitality of "Institut des Hautes Études Scientifiques" to which the author expresses his gratitude.

Some notation and terminology. Let $X$ be a $G$-space. Then $G_{x}$ denotes the isotropy subgroup of $G$ at the point $x \in X$. Let $H$ denote a subgroup of $G$, then 
$X^{H}$ denotes the fixed point set of $H$ and by $X^{>H}$ we denote the set of points with isotropy subgroup strictly greater than $H$, i.e., $X^{>H}=\left\{x \in X \mid H \varsubsetneqq G_{x}\right\}$. Thus $X^{H}-X^{>H}$ is the set of all points in $X$ with isotropy subgroup equal to $H$. Sometimes we also denote this set by $X_{H}$, i.e.,

$$
X_{H}=X^{H}-X^{>H} \text {. }
$$

A $G$-map or an equivariant map $f: X \rightarrow Y$, where $X$ and $Y$ are $G$-spaces, is a map such that $f(g x)=g f(x)$, for every $x \in X$ and all $g \in G$. It follows that $G_{x} \subset G_{f(x)}$, for every $x \in X$. An isovariant map $f: X \rightarrow Y$ is defined to be a $G$-map such that $G_{x}=G_{f(x)}$, for every $x \in X$.

Assume that $d$ is an invariant metric for $Y$. Let $\varepsilon: X \rightarrow \mathbf{R}$ be an invariant positive function (given a positive function $\varepsilon^{\prime}: X \rightarrow \mathbf{R}$, the function $\varepsilon: X \rightarrow \mathbf{R}$, defined by $\varepsilon(x)=\min _{g \in G} \varepsilon^{\prime}(g x)$, is an invariant positive function and $\varepsilon(x) \leqslant \varepsilon^{\prime}(x)$ for every $x \in X)$. Let $f: X \rightarrow Y$ be a $G$-map. A $G$-map $h: X \rightarrow Y$ is an $\varepsilon$-approximation to $f$ if $d(f(x), h(x))<\varepsilon(x)$ for every $x \in X$. A $G$-homotopy $F: X \times I \rightarrow Y$ from $f$ to $h$ is an $\varepsilon$-homotopy from $f$ to $h$ if $d(f(x), F(x, t))<\varepsilon(x)$ for every $x \in X$, i.e. each $F_{t}$ : $X \rightarrow Y$, where $F_{t}(x)=F(x, t)$, is an $\varepsilon$-approximation to $f$. If $F$ is an $\varepsilon$-homotopy we also have the symmetric condition $d(F(x, s), F(x, t))<2 \varepsilon(x)$ for every $x \in X$. Given an invariant positive function $\tilde{\varepsilon}: X \times I \rightarrow \mathbf{R}$ define $\varepsilon_{0}(x)=\min _{t \in I} \tilde{\varepsilon}(x, t)$. Then $\varepsilon: X \times I \rightarrow \mathbf{R}$, defined by $\varepsilon(x, t)=\varepsilon_{0}(x)$, is an invariant positive function and $\varepsilon(x, t) \leqslant \tilde{\varepsilon}(x, t)$ for all $(x, t) \in X \times I$. By \| \| we denote the standard norm in $\mathbf{R}^{n}$, i.e., $\|x\|=\sqrt{x_{1}^{2}+\ldots+x_{n}^{2}}$ for $x=\left(x_{1}, \ldots, x_{n}\right)$.

Let $A \subset \mathbf{R}^{n}$ and $x_{1}, \ldots, x_{r} \in \mathbf{R}^{n}$, then $\left\langle\left\langle A, x_{1}, \ldots, x_{r}\right\rangle\right\rangle$ denotes the affine subspace of $\mathbf{R}^{n}$ generated by the set $A \cup\left\{x_{1}, \ldots, x_{r}\right\}$. By Int $B$ we denote the interior of a set $B$, and int $M=M-\partial M$ denotes the interior of a manifold $M$ with boundary $\partial M$. A proper map is one for which the inverse image of any compact set is compact. In this paper the dimension of the empty set is somewhat problematic. Given the empty set $\varnothing$ we set $\operatorname{dim} \varnothing=-1$, but in other cases we may have a conclusion of the form $\operatorname{dim} A \leqslant-5$ and the correct conclusion to be drawn is that $A=\varnothing$. Further definitions and terminology are given in $\S 1$.

1. Preliminaries. Let $G$ be a finite group. By a simplicial complex we mean a geometric simplicial complex, that is, the topological realization of an abstract simplicial complex considered as a topological space together with the structure given by the simplexes. In this paper all simplicial complexes will be countable, locally finite and finite-dimensional. A simplicial $G$-complex consists of a simplicial complex $X$ together with a $G$-action $\varphi: G \times X \rightarrow X$ such that the map $g: X \rightarrow X$ is a simplicial homeomorphism for every $g \in G$. We define an equivariant simplicial complex to be a simplicial $G$-complex $X$ which satisfies the following two conditions.

1. For every subgroup $H$ of $G$ we have that if $s=\left\langle v_{0}, \ldots, v_{m}\right\rangle$ is a simplex of $X$ and $s^{\prime}=\left\langle h_{0} v_{0}, \ldots, h_{m} v_{m}\right\rangle$, where $h_{i} \in H, i=0, \ldots, m$, also is a simplex of $X$ then there exists $h \in H$ such that $h v_{i}=h_{i} v_{i}, i=0, \ldots, m$.

2. For any simplex $s$ of $X$ the vertices $v_{0}, \ldots, v_{n}$ of $s$ can be ordered in such a way that we have $G_{v_{n}} \subset \cdots \subset G_{v_{0}}$. 
In condition 1 the vertices $v_{0}, \ldots, v_{m}$ need not be distinct. The subgroup $G_{v_{n}}$, in condition 2, is called the principal isotropy subgroup of the simplex $s$ and $G_{v_{0}}$ the maximal isotropy subgroup of $s$. An abstract simplicial $G$-complex satisfying condition 1 above is called regular by Bredon [2, p. 116, Definition 1.2]. Now consider the following condition.

$1^{\prime}$. If $s=\left\langle v_{0}, \ldots, v_{m}\right\rangle$ is a simplex of $X$ and $s^{\prime}=\left\langle g_{0} v_{0}, \ldots, g_{m} v_{m}\right\rangle$, where $g_{i} \in G, i=0, \ldots, m$, also is a simplex of $X$ then there exists $g \in G$ such that $g v_{i}=g_{i} v_{i}, i=0, \ldots, m$.

We have

LeMmA 1.1. A simplicial G-complex satisfies conditions 1 and 2 if and only if it satisfies conditions $\mathbf{1}^{\prime}$ and 2.

Proof. Let $X$ be a simplicial $G$-complex which satisfies conditions $1^{\prime}$ and 2 . Let $H$ be a subgroup of $G$. Let $s=\left\langle v_{0}, \ldots, v_{m}\right\rangle$ be a simplex of $X$ and assume that $s^{\prime}=\left\langle h_{0} v_{0}, \ldots, h_{m} v_{m}\right\rangle$, where $h_{i} \in H, i=0, \ldots, m$, also is a simplex of $X$. We shall show that there exists $h \in H$ such that $h v_{i}=h_{i} v_{i}, i=0, \ldots, m$.

Since $X$ satisfies condition 2 we can assume that $v_{0}, \ldots, v_{m}$ are ordered such that $G_{v_{m}} \subset \cdots \subset G_{v_{0}}$. Since $X$ satisfies condition $1^{\prime}$ there exists $g \in G$ such that $g v_{i}=h_{i} v_{i}, i=0, \ldots, m$. Thus we have $h_{i}=g g_{i}$, where $g_{i} \in G_{v_{i}}, i=0, \ldots, m$. It now follows that

$$
h_{i}^{-1} h_{m}=\left(g g_{i}\right)^{-1} g g_{m}=g_{i}^{-1} g^{-1} g g_{m}=g_{i}^{-1} g_{m} \in G_{v_{i}}
$$

since $g_{m} \in G_{v_{m}} \subset G_{v_{i}}$. Thus $\left(h_{i}^{-1} h_{m}\right) v_{i}=v_{i}$, i.e., $h=h_{m} \in H$ is such that $h v_{i}=h_{i} v_{i}$, $i=0, \ldots, m$.

EXAMPLES. (a) We first describe a simplicial $G$-complex which satisfies condition $1^{\prime}$ but does not satisfy condition 1 . Let $G=Z_{2} \times Z_{2}$ and let $e, T \in Z_{2}$ denote the identity and generator, respectively, of $\mathbf{Z}_{2}$. Let $G$ act on $\mathbf{R}^{2}$ by $(T, e)(x, y)=$ $(-x, y)$ and $(e, T)(x, y)=(x,-y)$, and hence $(T, T)(x, y)=(-x,-y)$. Now define

$$
X=\left\{(x, y) \in \mathbf{R}^{2} \mid\|x\|+\|y\|=1\right\}
$$

and give $X$ the simplicial structure with vertices $v_{0}=(1,0), v_{1}=(0,1), v_{2}=(-1,0)$ and $v_{3}=(0,-1)$, and 1 -simplexes $\left\langle v_{0}, v_{1}\right\rangle,\left\langle v_{1}, v_{2}\right\rangle,\left\langle v_{2}, v_{3}\right\rangle$ and $\left\langle v_{3}, v_{0}\right\rangle$. Then $X$ is a simplicial $G$-complex which satisfies condition $1^{\prime}$. Now consider the subgroup $H=\{(e, e),(T, T)\}$ of $G$ and denote $\tau=(T, T)$. Then $\left\langle v_{0}, v_{1}\right\rangle$ and $\left\langle\tau v_{0}, v_{1}\right\rangle$ are simplexes of $X$ but there does not exist $h \in H$ such that $h v_{0}=\tau v_{0}$ and $h v_{1}=v_{1}$. Hence $X$ does not satisfy condition 1 .

(b) A simplicial $G$-complex $X$ which satisfies condition 1, i.e., is regular, but does not satisfy condition 2 can be constructed as follows. Let $G=\mathbf{Z}_{6}$, and let $X$ be the 1-dimensional simplicial $G$-complex whose 0-skeleton is $\mathbf{Z}_{6} / \mathbf{Z}_{3} \cup \mathbf{Z}_{6} / \mathbf{Z}_{2}$ with the natural $\mathbf{Z}_{6}$-action and 1-skeleton consisting of the six 1 -simplexes $a, T a, \ldots, T^{5} a$, where $a$ is a 1 -simplex joining the vertex $e \mathbf{Z}_{3}$ with the vertex $e \mathbf{Z}_{2}$.

(c) The simplicial complex $X$ of Example (a) with $\mathbf{Z}_{2}$-action given by the antipodal map is an example of a simplicial $\mathbf{Z}_{2}$-complex which satisfies condition 2 but does not satisfy condition 1 '. 
For a simplicial $G$-complex to be an equivariant simplicial complex is a purely technical condition. The second barycentric subdivision $X^{\prime \prime}$ of a simplicial $G$-complex $X$ satisfies condition 1 (see Bredon [2, p. 116, Proposition 1.1]) and it is also easily seen that $X^{\prime \prime}$ satisfies condition 2 . Thus we have

Lemma 1.2. Let $X$ be a simplicial G-complex. Then the second barycentric subdivision $X^{\prime \prime}$ is an equivariant simplicial complex.

Also observe that any equivariant subdivision of an equivariant simplicial complex is again an equivariant simplicial complex. Moreover observe that an equivariant simplicial complex in particular satisfies the following. For any simplex $s$ of $X$ we have that if $x \in s$ and $g x \in s$ then $g x=x$, and moreover that if $x \in s$ and $g x=x$ then $g y=y$ for every $y \in s$. Furthermore we have that if $x, y \in s$ then the isotropy subgroups $G_{x}$ and $G_{y}$ are in relation, i.e., either $G_{x} \subset G_{y}$ or $G_{y} \subset G_{x}$.

The orbit space $X / G$ of an equivariant simplicial complex $X$ has the structure of an ordinary simplicial complex such that the natural projection $p: X \rightarrow X / G$ is simplicial and $p$ maps each simplex of $X$ homeomorphically onto the corresponding image simplex of $X / G$.

Let $X$ be an equivariant simplicial complex and $Y$ an equivariant subcomplex of $X$. Recall that $Y$ is said to be full in $X$ if any simplex of $X$ all of whose vertices lie in $Y$ is a simplex of $Y$. We now define

Definition 1.3. An equivariant subcomplex $Y$ of $X$ is strongly full in $X$ if $Y$ is full in $X$ and satisfies the following condition: Let $s$ be a simplex of $X$ and $v$ a vertex of $s$ such that $v \in Y$. Then for any vertex $w$ of $s$ such that $G_{v} \varsubsetneqq G_{w}$ we also have $w \in Y$.

Let $H_{1}, \ldots, H_{s}$ be subgroups of $G$ and consider the equivariant subcomplex $\cup_{i=1}^{s} G X^{H_{i}}$ of $X$. Since $X$ is an equivariant simplicial complex it follows that $\bigcup_{i=1}^{s} G X^{H_{i}}$ is full in $X$. For let $s$ be a simplex of $X$ all of whose vertices lie in $\cup_{i=1}^{s} G X^{H_{i}}$, and let $w$ be a vertex of $s$ such that $G_{w}$ is the principal isotropy subgroup of $s$. Then $w \in G X^{H_{i_{0}}}$, some $i_{0}, 1 \leqslant i_{0} \leqslant s$, and it then follows that $s \subset G X^{H_{i_{0}}} \subset \cup_{i=1}^{s} G X^{H_{i}}$.

LEMMA 1.4. Let $Y$ be a strongly full subcomplex of the equivariant simplicial complex $X$. Then $Y \cup\left(\cup_{i=1}^{s} G X^{H_{i}}\right)$ is full in $X$, for any subgroups $H_{1}, \ldots, H_{s}$ of $G$. Conversely, let $Y$ be full in $X$ and assume that $Y \cup G X^{H}$ is full in $X$, for every subgroup $H$ of $G$, then $Y$ is strongly full in $X$.

Proof. Assume that $Y$ is strongly full in $X$ and let $H_{1}, \ldots, H_{s}$ be subgroups of $G$. Let $s$ be a simplex of $X$ with vertices $v_{0}, \ldots, v_{n} \in Y \cup\left(\cup_{i=1}^{s} G X^{H_{i}}\right)$. Let $v_{0}, \ldots, v_{m}$, where $-1 \leqslant m \leqslant n$, be all the vertices of $s$ that belong to $\cup_{i=1}^{s} G X^{H_{i}}$, thus $v_{m+1}, \ldots, v_{n} \in Y$. If $m=n$ it follows, since $\cup_{i=1}^{s} G X^{H_{i}}$ is full in $X$, that $s$ is a simplex of $\cup_{i=1}^{s} G X^{H_{i}}$ and hence also of $Y \cup\left(\cup_{i=1}^{s} G X^{H_{i}}\right)$. If $m=-1$ it follows that $s$ is a simplex of $Y$. Now assume $0 \leqslant m \leqslant n-1$. Then $v_{0} \in$ $\cup_{i=1}^{s} G X^{H_{i}}$, say $v_{0} \in G X^{H_{i_{0}}}$, and $v_{n} \in Y$. We have $G_{v_{0}} \subset G_{v_{n}}$ or $G_{v_{n}} \subset G_{v_{0}}$. If $G_{v_{0}} \subset G_{v_{n}}$ then $v_{n} \in G X^{H_{i_{0}}} \subset \cup_{i=1}^{s} G X^{H_{i}}$ which is a contradiction since $m<n$. Thus $G_{v_{n}} \varsubsetneqq G_{v_{0}}$, and since $Y$ is strongly full it follows that $v_{0} \in Y$. In this way we 
see that $v_{0}, \ldots, v_{m} \in Y$, and thus it follows that $s$ is a simplex of $Y$ and hence also of $Y \cup\left(\cup_{i=1}^{s} G X^{H_{i}}\right)$.

Now let $Y$ be full in $X$ and assume that $Y \cup G X^{H}$ is full in $X$ for every subgroup $H$ of $G$. Let $s$ be a simplex of $X$, and let $v$ be a vertex of $s$ which lies in $Y$. Let $w$ be some other vertex of $s$ with $G_{v} \varsubsetneqq G_{w}$. Let $\sigma$ be the 1-simplex with vertices $v$ and $w$. Then $v \in Y \cup G X^{G_{w}}$ and $w \in Y \cup G X^{G_{w}}$ and hence, by our assumption that $Y \cup G X^{G_{w}}$ is full, we have $\sigma \subset Y \cup G X^{G_{w}}$. Since $\sigma \notin G X^{G_{w}}$ we have $\sigma \subset Y$, and hence also $w \in Y$, which is what we wished to prove.

Also observe that the condition of being a strongly full subcomplex is a purely technical condition, for if $Y$ is an equivariant subcomplex of $X$ we have by taking the first barycentric subdivision that $Y^{\prime}$ is strongly full in $X^{\prime}$. Moreover we have that if $Y$ is full or strongly full in $X$ then $Y^{*}$ is full or strongly full, respectively, in $X^{*}$, for any equivariant subdivision $X^{*}$ of $X$.

Let $\rho: G \rightarrow O(m)$ be an orthogonal representation of $G$. By $\mathbf{R}^{m}(\rho)$ we denote euclidean space $\mathbf{R}^{m}$ together with $G$-action through $\rho$. We denote

$$
\begin{gathered}
D^{m}(\rho)=\text { convex hull of }\left\{ \pm g e_{i} \mid g \in G, i=1, \ldots, m\right\}, \\
\qquad S^{m-1}(\rho)=\partial D^{m}(\rho) .
\end{gathered}
$$

Here $e_{1}, \ldots, e_{m}$ denote the standard unit vectors in $\mathbf{R}^{m}$. The $G$-spaces $S^{m-1}(\rho)$, $D^{m}(\rho)$ and $\mathbf{R}^{m}(\rho)$ can be triangulated such that they are equivariant simplicial complexes, and such that $S^{m-1}(\rho)$ is an equivariant subcomplex of $D^{m}(\rho)$ and $D^{m}(\rho)$ is an equivariant subcomplex of $\mathbf{R}^{m}(\rho)$, see [8, $\left.\S 1\right]$. Also recall that if $K_{1}$ and $K_{2}$ are rectilinear equivariant simplicial complexes in $\mathbf{R}^{m}(\rho)$ such that $\left|K_{1}\right|=\left|K_{2}\right|$ then $K_{1}$ and $K_{2}$ have a common equivariant (simplicial!) subdivision, see [8, $\left.\$ 1\right]$. We will need the following lemma.

Lemma 1.5. Let $X$ be a finite equivariant simplicial complex and $f: X \rightarrow D^{m}(\rho) a$ linear G-map. Then there exists an equivariant subdivision $X^{\prime}$ of $X$ and an equivariant triangulation $D^{m}(\rho)_{t}$ of $D^{m}(\rho)$, such that $f: X^{\prime} \rightarrow D^{m}(\rho)_{t}$ is a simplicial G-map.

Proof. For the proof it is convenient to have $X$ imbedded as a rectilinear equivariant simplicial complex in some $\mathbf{R}^{n}(\tau)$ (such an embedding exists by Proposition 1.1 in [8]). Let $D^{m}(\rho)_{t_{0}}$ be an equivariant triangulation of $D^{m}(\rho)$, i.e., $D^{m}(\rho)_{t_{0}}$ denotes a rectilinear equivariant simplicial complex structure on $D^{m}(\rho)$. Let $X^{*}$ be an ordinary subdivision of $X$ and $D^{m}(\rho)_{t_{0}}^{*}$ an ordinary subdivision of $D^{m}(\rho)_{t_{0}}$ such that $f: X^{*} \rightarrow D^{m}(\rho)_{t_{0}}^{*}$ is simplicial. For each $g \in G$ we have a new rectilinear ordinary simplicial complex structure, denoted $g D^{m}(\rho)_{t_{0}}^{*}$, on $D^{m}(\rho)$. The simplexes of $g D^{m}(\rho)_{t_{0}}^{*}$ are of the form $g \sigma$, where $\sigma$ denotes some simplex of $D^{m}(\rho)_{t_{0}}^{*}$. The intersection of all the rectilinear simplicial complex structures $g D(\rho)_{t_{0}}^{*}, g \in G$, gives us a rectilinear cell $G$-complex structure, denoted $\tilde{D}^{m}(\rho)$, on $D^{m}(\rho)$. The cells of $\tilde{D}^{m}(\rho)$ are of the form $c=\cap{ }_{i=1}^{N} g_{i} \sigma_{i}$, where $N=|G|$ and $g_{i} \neq g_{i}$, if $i \neq i^{\prime}$, and each $\sigma_{i}$ is a simplex of $D^{m}(\rho)_{t_{0}}^{*}, i=1, \ldots, N$. Since $g\left(\cap_{i=1}^{N} g_{i} \sigma_{i}\right)=\cap_{i=1}^{N}\left(g g_{i}\right) \sigma_{i}$ we have that for each cell $c$ of $\tilde{D}^{m}(\rho)$ also gc is a cell of $\tilde{D}^{m}(\rho)$, for every $g \in G$. Now $\tilde{D}^{m}(\rho)$ has a $G$-subdivision $D^{m}(\rho)_{t}$, obtained by inductively starring the cells of $\tilde{D}^{m}(\rho)$ at the centroids (beginning with the 2-cells), which is a simplicial 
$G$-complex. (In connection with the above argument see Edmonds [4].) Since $D^{m}(\rho)_{t_{0}}$ is an equivariant simplicial complex and $D^{m}(\rho)_{t}$ is a (simplicial) $G$-subdivision of $D^{m}(\rho)_{t_{0}}$ it follows that $D^{m}(\rho)_{t}$ is in fact an equivariant simplicial complex.

Let $\tilde{X}$ be the rectilinear cell $G$-complex structure on $|X|$ which is the intersection of all the rectilinear simplicial complex structures $g X^{*}, g \in G$, as above. Then $f$ : $\tilde{X} \rightarrow \tilde{D}^{m}(\rho)$ maps each cell $d$ of $\tilde{X}$ onto a cell $f(d)$ of $\tilde{D}^{m}(\rho)$. Now let $X^{\prime}$ be a $G$-subdivision of $\tilde{X}$ obtained by starring each cell $d(\operatorname{dim} d \geqslant 2)$ at some point $\hat{d} \in \stackrel{d}{d}$ for which $f(\hat{d})=\beta(f(d))$, where $\beta(f(d))$ denotes the centroid of the cell $f(d)$, and moreover in such a way that the cell $g d, g \in G$, is starred at the point $g \hat{d}$. To see that we can in fact have this last condition satisfied observe that the rectilinear cell $G$-complex $\tilde{X}$ has the property that, if $g d=d$ for some cell $d$ of $\tilde{X}$ and $g \in G$, then $g x=x$ for every $x \in d$. This property of $\tilde{X}$ follows from the fact that $\tilde{X}$ is a $G$-subdivision of the equivariant simplicial complex $X$. Hence the process of starring each cell $g d, g \in G$, at $g \hat{d}$ is well defined, for if $g_{1} d=g_{2} d$ then $g_{1} x=g_{2} x$ for every $x \in d$, and thus in particular $g_{1} \hat{d}=g_{2} \hat{d}$. Then $X^{\prime}$ is a simplicial $G$-complex, and since $X^{\prime}$ is a $G$-subdivision of $X$ we have in fact that $X^{\prime}$ is an equivariant simplicial complex. Moreover the map $f: X^{\prime} \rightarrow D^{m}(\rho)_{t}$ is simplicial.

LeMma 1.6. Let $K$ and $L$ be equivariant simplicial complexes and $f: K \rightarrow L a$ proper G-map which is p.l. Then there exist equivariant subdivisions $K^{\prime}$ and $L^{\prime}$ of $K$ and $L$, respectively, such that $f: K^{\prime} \rightarrow L^{\prime}$ is a simplicial G-map.

Proof. Since $f: K \rightarrow L$ is a proper p.l. map there exist ordinary subdivisions $K^{*}$ and $L^{*}$ of $K$ and $L$ respectively such that $f: K^{*} \rightarrow L^{*}$ is simplicial. The rest of the proof is completely analogous to the proof of Lemma 1.5 .

In the following Lemma 1.6 will be used without specific reference to it. In a way one wishes to define a proper map $f: K \rightarrow L$ to be a p.l. $G$-map if there exist equivariant subdivisions $K^{\prime}$ and $L^{\prime}$ of $K$ and $L$, respectively, such that $f: K^{\prime} \rightarrow L^{\prime}$ is a simplicial $G$-map, and Lemma 1.6 then shows that this definition is well defined in the sense that a proper map $f: K \rightarrow L$ is a p.l. $G$-map if and only if it is a p.l. map and a $G$-map.

The notion of an equivariant combinatorial manifold, with or without boundary, is also defined in [8] but for completeness we repeat it here. An equivariant simplicial complex $M$ is an $m$-dimensional equivariant combinatorial manifold if for every vertex $v$ of $M$ there exist an orthogonal representation $\rho_{v}: G_{v} \rightarrow O(m)$ and a p.l. $G_{v}$-homeomorphism

$$
\alpha: \operatorname{Lk}(v, M) \rightarrow S^{m-1}\left(\rho_{v}\right) .
$$

We say that $M$ is an $m$-dimensional equivariant combinatorial manifold with boundary if for every vertex $v$ of $M$ either $\operatorname{Lk}(v, M)$ is p.l. $G_{v}$-homeomorphic with $S^{m-1}\left(\rho_{v}\right)$, for some $\rho_{v}: G_{v} \rightarrow O(m)$, as above, or there exist an orthogonal representation $\rho_{v}^{\prime}: G_{v} \rightarrow O(m-1)$ and a p.l. $G_{v}$-homeomorphism

$$
\alpha^{\prime}: \operatorname{Lk}(v, M) \rightarrow D^{m-1}\left(\rho_{v}^{\prime}\right) .
$$

In particular an equivariant combinatorial manifold $M$, with or without boundary, is when we forget the $G$-action a combinatorial manifold, with or without 
boundary, respectively. We denote the boundary of $M$ by $\partial M$. Also observe that in the case that $\operatorname{Lk}(v, M)$ is p.l. $G_{v}$-homeomorphic with $S^{m-1}\left(\rho_{v}\right)$ there exists a p.l. $G_{v}$-homeomorphism $\beta: \operatorname{St}(v, M) \rightarrow D^{m}\left(\rho_{v}\right)$, and if $\operatorname{Lk}(v, M)$ is p.l. $G_{v}$-homeomorphic with $D^{m-1}\left(\rho_{v}^{\prime}\right)$ there exists a p.l. $G_{v}$-homeomorphism

$$
\beta: \operatorname{St}(v, M) \rightarrow D^{m}\left(\rho_{v}^{\prime} \oplus \text { id }\right) \text {. }
$$

For any $x \in \partial M$ we have, if $x \in \operatorname{St}(v, M)$ and $\beta: \operatorname{St}(v, M) \rightarrow D^{m}\left(\tau_{v}\right)$ is a p.l. $G_{v}$-homeomorphism, that $\beta(x) \in \partial D^{m}\left(\tau_{v}\right)$.

Also note that in [8] it is proved that if $N$ is a smooth $G$-manifold, with or without boundary, and $h: K \rightarrow N$ is a smooth equivariant triangulation of $N$, then $K$ is an equivariant combinatorial manifold, with or without boundary, respectively. Moreover existence and combinatorial uniqueness of smooth equivariant triangulations of any smooth $G$-manifold $N$, with or without boundary, is established in [8].

Let $S$ be a $G$-space. An equivariant triangulation of $S$ consists of an equivariant simplicial complex $K$ and a $G$-homeomorphism $t: K \rightarrow S$. Two equivariant triangulations $t_{1}: K_{1} \rightarrow S$ and $t_{2}: K_{2} \rightarrow S$ are called equivalent if $t_{2}^{-1} t_{1}: K_{1} \rightarrow K_{2}$ is a p.l. $G$-homeomorphism. Given an equivariant triangulation $t: K \rightarrow S$ we have the class of all equivariant triangulations of $S$ equivalent to $t$, called the preferred class of equivariant triangulations of $S$ determined by $t$. Two equivariant triangulations $t_{1}$ and $t_{2}$ of $S$ determine the same class of preferred triangulations of $S$ if and only if $t_{1}$ and $t_{2}$ are equivalent. An equivariant polyhedron $P$ is a $G$-space $P$ together with a (nonempty!) preferred class of equivariant triangulations of $P$. An equivariant subpolyhedron $Q$ of $P$ is a $G$-subset of $P$ such that for some preferred equivariant triangulation $h: K \rightarrow P$ there exists an equivariant subcomplex $L$ of $K$ such that $h(L)=Q$. In particular it follows that $Q$ is a closed subset of $P$. An $m$-dimensional equivariant p.l. manifold (with or without boundary) $M$ is an equivariant polyhedron such that in the class of preferred equivariant triangulations of $M$ there exists an equivariant triangulation $t: K \rightarrow M$ with $K$ an $m$-dimensional equivariant combinatorial manifold (with or without boundary). In fact, in this case, for any preferred equivariant triangulation $t^{\prime}: L \rightarrow M$ we have that $L$ is an $m$-dimensional equivariant combinatorial manifold (with or without boundary). In this paper we shall from now on use the terms "equivariant combinatorial manifold $M$ " and "equivariant p.l. manifold $M$ " both in case $\partial M=\varnothing$ and in case $\partial M \neq \varnothing$.

Let $P$ and $Q$ be equivariant polyhedra. A proper map $f: P \rightarrow Q$ is a p.l. $G$-map if the proper map $\tau^{-1} \circ f \circ t: K \rightarrow L$ is a p.l. $G$-map for some (and hence all) equivariant triangulations $t: K \rightarrow P$ and $\tau: L \rightarrow Q$ of $P$ and $Q$, respectively.

Let us also note in this connection that given a proper $G$-map $f: P \rightarrow Q$ there exists an invariant positive function $\varepsilon: P \rightarrow \mathbf{R}$ such that any $\varepsilon$-approximation $h$ : $P \rightarrow Q$ to $f$ also is proper. It then follows by applying this to the proper map $F_{c}$ : $P \times I \rightarrow Q$, where $F_{c}(x, t)=f(x)$, that there in fact exists an invariant positive function $\varepsilon: P \rightarrow \mathbf{R}$ such that if $F: P \times I \rightarrow Q$ is any $\varepsilon$-homotopy from $f$ to some map $h$, then $F$ is a proper map.

Let $K$ be an equivariant simplicial complex and $S$ a $G$-space. We say that a $G$-map $f: K \rightarrow S$ is equivariantly nondegenerate if for every equivariant simplex $G s$ 
of $K$ we have that $f \mid: G s \rightarrow S$ is a $G$-imbedding. Observe that any equivariantly nondegenerate $G$-map is isovariant and nondegenerate in the ordinary sense, i.e., imbeds ordinary simplexes of $K$. The following example shows that the converse is not generally true.

EXAMPLE. Let $G=\mathrm{Z}_{3}=\left\{e, T, T^{2}\right\}$ and $K=\mathrm{Z}_{3} \times I$, with $\mathrm{Z}_{3}$-action given by left multiplication on $Z_{3}$ and trivial action on the unit interval $I$. Let $L=\partial \Delta^{2}$, the boundary of a 2 -simplex, with simplicial $\mathbf{Z}_{3}$-action given by permutation of the vertices $a, T a$ and $T^{2} a$. Then $L$ is a simplicial $G$-complex (but not an equivariant simplicial complex!). Let $f: K \rightarrow L$ be the $G$-map defined by $f(e, t)=(1-t) a+$ $t T a$, for any $t \in I$. Then $f$ is a simplicial $G$-map which is isovariant and nondegenerate, in the ordinary sense, but $f$ is not equivariantly nondegenerate. An analogous example, with a linear $G$-map from $K$ into $\mathbf{R}^{2}$ with $\mathbf{Z}_{3}$-action given by $T z=e^{2 \pi i / 3} z$, all $z \in \mathbf{R}^{2}$, shows that: A linear $G$-map from an equivariant simplicial complex into some orthogonal representation space that is isovariant and nondegenerate in the ordinary sense need not be equivariantly nondegenerate.

On the other hand we have

LEMMA 1.7. Let $K$ and $L$ be equivariant simplicial complexes and $f: K \rightarrow L$ a simplicial G-map that is isovariant and nondegenerate in the ordinary sense. Then $f$ is equivariantly nondegenerate.

Proof. Let $G s$ be an arbitrary equivariant simplex of $K$, where $s$ denotes an ordinary simplex of $K$. Let $a, b \in G s$ such that $f(a)=f(b)$. Write $a=g_{1} u$ and $b=g_{2} v$, where $u, v \in s$. Then $f\left(g_{1} u\right)=f\left(g_{2} v\right)$ and thus, denoting $g_{0}=g_{1}^{-1} g_{2}$, we have $f(u)=f\left(g_{0} v\right)$. Hence $f(u) \in f(s) \cap g_{0} f(s)$, and therefore, since $L$ is an equivariant simplicial complex, we have $g_{0} f(u)=f(u)$. This implies that $f(u)=f(v)$ and hence that $u=v$, since $f$ is nondegenerate. Moreover $g_{0} f(u)=f(u)$ implies that $g_{0} \in G_{f(u)}=G_{u}$, since $f$ is isovariant. Thus $g_{0} u=u$ and therefore $a=g_{1} u=g_{2} u=$ $g_{2} v=b$.

If $P$ is an equivariant polyhedron we say that a $G$-map $f: P \rightarrow S$ is equivariantly nondegenerate if for some equivariant simplicial complex $P^{*}$ triangulating $P$ the $G$-map $f: P^{*} \rightarrow S$ is equivariantly nondegenerate.

2. Local results. In this section we consider the local situation, i.e., we consider maps from a finite equivariant simplicial complex $X$ into some $D^{m}(\rho)$ and also general position of equivariant subcomplexes of $D^{m}(\rho)$. These results are then globalized in the next section to give the desired approximation results in the case of equivariant or isovariant maps from equivariant polyhedra into equivariant p.l. manifolds. In this section $X$ will always denote a finite equivariant simplicial complex. We begin with the following well-known lemma.

Lemma 2.1. Let $Y$ and $Z$ be equivariant subcomplexes of $X$ and let $f: X \rightarrow D^{m}(\rho)$ be a $G$-map such that $f \mid: Y \cap Z \rightarrow D^{m}(\rho)$ is linear. Then, given $\varepsilon>0$, there exist an equivariant subdivision $X^{\prime}$ of $X$ and a G-map $h: X^{\prime} \rightarrow D^{m}(\rho)$ such that $h \mid: Y^{\prime} \rightarrow$ $D^{m}(\rho)$ is linear and $h$ is equivariantly $\varepsilon$-homotopic rel $|Z|$ to $f$. 
Proof. Let $\delta>0$ be such that $d(x, y)<\delta$ implies $\|f(x)-f(y)\|<\varepsilon / 2$. Let $X^{\prime}$ be an equivariant subdivision of $X$ such that $(Y \cup Z)^{\prime}$ is full in $X^{\prime}$ and $\operatorname{mesh}\left(X^{\prime}\right)$ $<\delta$. Now define $h: X^{\prime} \rightarrow D^{m}(\rho)$ as follows. If $s=\left\langle v_{0}, \ldots, v_{k}\right\rangle$ is a simplex of $Y^{\prime}$ define $h$ on the vertices of $s$ by $h\left(v_{i}\right)=f\left(v_{i}\right), i=0, \ldots, k$, and then define $h$ on all of $s$ by extending linearly. If $s$ is a simplex of $Z^{\prime}$ define $h$ on $s$ by $h|s=f| s$. Since $f$ is a $G$-map and $f \mid(Y \cap Z)^{\prime}$ is linear it follows that this gives us a well-defined $G$-map $h:(Y \cup Z)^{\prime} \rightarrow D^{m}(\rho)$ such that $h \mid Y^{\prime}$ is linear and $h\left|Z^{\prime}=f\right| Z^{\prime}$.

Then extend $h$ to all of $X^{\prime}$ in the following way. If $s$ is a simplex of $X^{\prime}$ such that $s \cap|Y \cup Z|=\varnothing$ we define $h|s=f| s$. Now let $s$ be a simplex of $X^{\prime}-(Y \cup Z)^{\prime}$ such that $s \cap|Y \cup Z| \neq \varnothing$. Since $(Y \cup Z)^{\prime}$ is full in $X^{\prime}$ we have $s=s_{1} s_{2}$, where $s_{1} \cap|Y \cup Z|=\varnothing$ and $s_{2}$ is a simplex of $(Y \cup Z)^{\prime}$. Define $h$ on $s$ by extending linearly the map $h$ already defined on $s_{1}$ and $s_{2}$. This gives us a well-defined $G$-map $h: X^{\prime} \rightarrow D^{m}(\rho)$ such that $h \mid Y^{\prime}$ is linear and $h\left|Z^{\prime}=f\right| Z^{\prime}$.

It follows from the way the map $h$ is constructed that $\operatorname{diam}(h(s)) \leqslant \operatorname{diam}(f(s))<$ $\varepsilon / 2$, for every simplex $s$ of $X^{\prime}$, and hence, since $h$ and $f$ agree on all the vertices of $X^{\prime}$, we have $\|h(x)-f(x)\|<\varepsilon$ for every $x \in X$. The map $H$ defined by $H(x, t)=$ $(1-t) f(x)+t h(x)$ is an equivariant $\varepsilon$-homotopy rel $|Z|$ from $f$ to $h$.

Proposition 2.2. Let $Y_{1}, Y_{2}$ and $Y_{3}$ be equivariant subcomplexes of $X$ such that $Y_{1} \cap Y_{3}=\varnothing$ and let $f: X \rightarrow D^{m}(\rho)_{t}$ be a G-map such that $f \mid: Y_{2} \rightarrow D^{m}(\rho)_{t}$ is simplicial. Let $\varepsilon>0$ be given. Then there exist equivariant subdivisions $X^{*}$ and $D^{m}(\rho)_{t}^{*}$ of $X$ and $D^{m}(\rho)_{t}$, respectively, and a G-map $h: X^{*} \rightarrow D^{m}(\rho)_{t}^{*}$ such that $h \mid$ : $\left(Y_{1} \cup Y_{2}\right)^{*} \rightarrow D^{m}(\rho)_{t}^{*}$ is simplicial and $h$ is equivariantly $\varepsilon$-homotopic rel $\left|Y_{2} \cup Y_{3}\right|$ to $f$.

Proof. Let $Y=Y_{1} \cup Y_{2}$ and $Z=Y_{2} \cup Y_{3}$ and note that since $Y_{1} \cap Y_{3}=\varnothing$ we have $Y \cap Z=Y_{2}$. Now Proposition 2.2 follows easily from Lemma 2.1 using Lemma 1.5.

The next result (Lemma 2.3 and Proposition 2.4 below) proves that one can approximate isovariant maps by isovariant simplicial maps and this in a relative fashion. The proof of Lemma 2.3 is quite involved. There are also some fine points to observe. The first is that already Lemma 2.3 is given in the form with subcomplexes $Y_{1}, Y_{2}$ and $Y_{3}$ where $Y_{1} \cap Y_{3}=\varnothing$, which is a stronger assumption than the one of the type used in Lemma 2.1 with subcomplexes $Y$ and $Z$ (cf. the proof of Proposition 2.2). We have pointed out in the proof of Lemma 2.3 where this extra strength of the assumption is needed. The other point is that in establishing the existence of an isovariant map $h$, of desired type, that approximates the given isovariant map $f$ we only prove that $h$ is equivariantly homotopic to $f$. (This situation should be compared with the result in Lemma 2.7.)

Before giving Lemma 2.3 and its proof we shall here describe some notation and also a specific subdivision procedure that we use in the proof of Lemma 2.3. For any subgroup $H_{0}$ of $G$ we denote

$$
J\left(H_{0}\right)=\left\{H \text { is a subgroup of } G \mid H \nsubseteq H_{0}\right\} .
$$


We define

$$
B\left(H_{0}\right)=\bigcup_{H \in J\left(H_{0}\right)} D^{m}(\rho)^{H} .
$$

Then $B\left(H_{0}\right)$ is a closed subset of $D^{m}(\rho)$ and for any $x \in D^{m}(\rho)$ we have that $x \in D^{m}(\rho)-B\left(H_{0}\right)$ if and only if $G_{x} \subset H_{0}$. If $s$ is a simplex of some equivariant simplicial complex we denote by $I_{0}(s)$ the maximal isotropy subgroup of $s$. For any finite equivariant simplicial complex $K$ and any subgroup $H$ of $G$ we denote by $K$ $(\leqslant H)$ the ordinary subcomplex of $K$ consisting of all the simplexes $s$ (together with their faces) of $K$ that have $I_{0}(s)=H$, i.e., $K(\leqslant H)$ is an ordinary subcomplex of $K$ and

$$
K(\leqslant H)=\bigcup_{\substack{s<K \\ I_{0}(s)=H}} s .
$$

Let $L$ be an equivariant subcomplex of $K$ such that $L$ is full in $K$. By $N(L, K)$ we denote the simplicial neighborhood of $L$ in $K$, i.e., $N(L, K)$ is a subcomplex of $K$ and

$$
N(L, K)=\bigcup_{\substack{s<K \\ s \cap L \neq \varnothing}} s .
$$

Clearly $N(L, K)$ is an equivariant subcomplex of $K$ and

$$
\dot{N}(L, K)=\{s<N(L, K) \mid s \cap L=\varnothing\}
$$

is an equivariant subcomplex of $N(L, K)$. Since $L$ is full in $K$ we have that every simplex $s$ of $N(L, K)$ can be written in a unique way as a join $s=s_{1} s_{2}$ where $s_{1}<L$ and $s_{2}<\dot{N}(L, K)$. By $C(L, K)$ we denote the simplicial complement of $L$ in $K$, i.e.,

$$
C(L, K)=\bigcup_{\substack{s<K \\ s \cap L=\varnothing}} s .
$$

Then $C(K, L)$ is an equivariant subcomplex of $K$ and we have

$$
K=N(K, L) \cup C(L, K) \text { and } N(L, K) \cap C(L, K)=\dot{N}(L, K) .
$$

Now let $C(L, K)^{*}$ be any equivariant subdivision of $C(L, K)$. Then extend this subdivision to an equivariant subdivision $K^{*}$ of $K$ as follows. By $\dot{N}(L, K)^{*}$ we denote the equivariant subdivision of $\dot{N}(L, K)$ induced from the subdivision $C(L, K)^{*}$ of $C(L, K)$. If $s$ is a simplex of $N(L, K)$ we write $s=s_{1} s_{2}$ where $s_{1}<L$ and $s_{2}<\dot{N}(L, K)$. Let $s_{2}^{*}$ denote the subdivision of $s_{2}$ induced from $\dot{N}(L, K)^{*}$. Then define a subdivision $s^{*}$ of $s$ by $s^{*}=s_{1} s_{2}^{*}$. This gives us an equivariant subdivision of $N(L, K)$ which on $\dot{N}(L, K)$ equals $\dot{N}(L, K)^{*}$, and hence an equivariant subdivision $K^{*}$ of $K$ which is an extension of the subdivision $C(L, K)^{*}$ of $C(L, K)$. We also have $L^{*}=L$. We call $K^{*}$ the join extension of $C(L, K)^{*}$ to $K$. Observe that for the join extension $K^{*}$ of $C(L, K)^{*}$ to $K$ the following holds (this will be crucial for us). For any equivariant full subcomplex $L_{0}$ of $L$ we have $L_{0}^{*}=L_{0}$ and $N\left(L_{0}^{*}, K^{*}\right)=N\left(L_{0}, K\right)^{*}$ and $\dot{N}\left(L_{0}^{*}, K^{*}\right)=\dot{N}\left(L_{0}, K\right)^{*}$ and

$$
C\left(L_{0}^{*}, K^{*}\right)=C\left(L_{0}, K\right)^{*} \text {. }
$$


LEMMA 2.3. Let $Y_{1}, Y_{2}$ and $Y_{3}$ be equivariant subcomplexes of $X$ such that $Y_{1} \cap Y_{3}=\varnothing$ and let $f: X \rightarrow D^{m}(\rho)$ be an isovariant map such that $f \mid: Y_{2} \rightarrow D^{m}(\rho)$ is linear. Then, given $\varepsilon>0$, there exist an equivariant subdivision $X^{\prime}$ of $X$ and an isovariant map $h: X^{\prime} \rightarrow D^{m}(\rho)$ such that $h \mid:\left(Y_{1} \cup Y_{2}\right)^{\prime} \rightarrow D^{m}(\rho)$ is linear and $h$ is equivariantly e-homotopic rel $\left|Y_{2} \cup Y_{3}\right|$ to $f$.

Proof. Before giving the actual proof let us heuristically describe the idea in the proof. Let $\tilde{s}$ be an ordinary simplex of $X$ and let $H=I_{0}(\tilde{s})$ be the maximal isotropy subgroup of $\tilde{s}$. Since $f: X \rightarrow D^{m}(\rho)$ is isovariant we have

$$
f(\tilde{s}) \subset D^{m}(\rho)-B(H) .
$$

Thus $\operatorname{dist}(f(\tilde{s}), B(H))>0$. The first part of the proof consists of constructing an equivariant subdivision $X^{\prime}$ of $X$ such that denoting

$$
d_{s}=\operatorname{dist}\left(f(s), B\left(I_{0}(s)\right)\right)
$$

we have

$$
\operatorname{diam} f(s)<\frac{1}{2} \min \left(d_{s}, \varepsilon\right),
$$

for every simplex $s$ of $X^{\prime}$. Once this has been done the map $h$ is constructed so that it agrees with $f$ on all vertices of $X^{\prime}$ and is linear in $\left(Y_{1} \cup Y_{2}\right)^{\prime}$. By induction, in the dimension of simplexes, it then follows using (*) that $h$ is isovariant.

Now let us say a few words about the construction of the subdivision $X^{\prime}$ of $X$. Let for example $s=\left\langle v_{0}, v_{1}, v_{2}\right\rangle$ be a 2-simplex with $G_{v_{0}}=H_{0}$ and $G_{v_{1}}=G_{v_{2}}=H_{1}$ where $H_{0} \supsetneq H_{1}$, and let $s_{1}=\left\langle v_{1}, v_{2}\right\rangle$ be the back face of $s$. Assume that we have $\operatorname{diam} f(s)<\frac{1}{2} \min \left(d_{s}, \varepsilon\right)$, where $d_{s}=\operatorname{dist}\left(f(s), B\left(H_{0}\right)\right)$, but $\operatorname{diam} f\left(s_{1}\right) \nless \frac{1}{2} \min \left(d_{s_{1}}, \varepsilon\right)$, where $d_{s_{1}}=\operatorname{dist}\left(f\left(s_{1}\right), B\left(H_{1}\right)\right)$. Thus we need to subdivide further in order to make $s_{1}$ and hence also $f\left(s_{1}\right)$ smaller. But we must not introduce new simplexes, with maximal isotropy subgroup equal to $H_{1}$, whose images under $f$ come closer to $B\left(H_{1}\right)$ than $f\left(s_{1}\right)$. Hence we may not introduce any new vertices in $s$ that lie outside $s_{1}$. Thus we subdivide $s$ by taking an appropriate subdivision $s_{1}^{\prime}$ of $s_{1}$ and give $s$ the subdivision $s^{\prime}=v_{0} s_{1}^{\prime}$. This is the main idea behind the construction of the equivariant subdivision $X^{\prime}$ of $X$ which satisfies (*).

Let us now begin the actual proof. We shall first construct an equivariant subdivision $X^{\prime}$ of $X$, with $Y^{\prime}$ full in $X^{\prime}$, such that for any simplex $s$ of $X^{\prime}$ we have

$$
\operatorname{diam} f(s)<\frac{1}{2} \min \left(d_{s}, \varepsilon\right)
$$

where

$$
d_{s}=\operatorname{dist}\left(f(s), B\left(I_{0}(s)\right)\right) \text {. }
$$

Let $H_{1}, \ldots, H_{r}$ be subgroups of $G$ forming a complete set of representatives for the orbit types occurring in $X$, ordered in such a way that $\left(H_{i}\right)>\left(H_{j}\right)$ implies $i<j$. We denote

$$
X_{i}=\bigcup_{j=1}^{i} G X^{H_{j}}, \quad i=1, \ldots, r
$$

and define $X_{0}=\varnothing$. Let $X^{(0)}$ be the first barycentric subdivision of $X$, then $Y_{1}^{(0)}$ is strongly full in $X^{(0)}$, and this property is then preserved under further subdivisions 
of $X^{(0)}$. The fact that each $X_{i}^{(0)}$ is full in $X^{(0)}$ (in fact $X_{i}$ is full in $X$ ) and that this property is preserved under further subdivisions of $X^{(0)}$ will also be used in the proof. We shall inductively construct successive equivariant subdivisions $X^{(0)}$, $X^{(1)}, \ldots, X^{(r)}$ of $X^{(0)}$, and equivariant subcomplexes $A_{1}^{(0)}, A_{2}^{(1)}, \ldots, A_{r+1}^{(r)}$ such that the following conditions are satisfied

(a) $A_{i+1}^{(n)}=C\left(X_{i}^{(n)}, X^{(n)}\right), 0 \leqslant i \leqslant n, n=0, \ldots, r$,

(b) $\operatorname{mesh}\left(A_{n}^{(n)}\right)<\delta_{n}, n=1, \ldots, r$,

where $\delta_{n}>0$ is such that $d(x, y)<\delta_{n}$, any $x, y \in X$, implies that

$$
\|f(x)-f(y)\|<\frac{1}{2} \min \left(\operatorname{dist}\left(f\left(A_{n}^{(n-1)}\left(\leqslant H_{n}\right)\right), B\left(H_{n}\right)\right), \varepsilon\right) .
$$

We have already constructed $X^{(0)}$. Now let $A_{1}^{(0)}=X^{(0)}$. Then condition (a) is satisfied and condition (b) is satisfied in an empty way. Now assume that $X^{(0)}, \ldots, X^{(n)}$ and $A_{1}^{(0)}, \ldots, A_{n+1}^{(n)}$, where $0 \leqslant n$, have been constructed and satisfy conditions (a) and (b). Since we have $\varnothing=\left|X_{0}\right| \subset\left|X_{1}\right| \subset \cdots \subset\left|X_{r}\right|=|X|$ it follows from the formula

$$
A_{i+1}^{(n)}=C\left(X_{i}^{(n)}, X^{(n)}\right), \quad 0 \leqslant i \leqslant n,
$$

that

$$
|X|=\left|A_{1}\right| \supset\left|A_{2}\right| \supset \cdots \supset\left|A_{n+1}\right|
$$

and also that the orbit types occurring in $\left|A_{i+1}\right|$, where $0 \leqslant i \leqslant n$, are $\left(H_{i+1}\right), \ldots,\left(H_{r}\right)$. Now consider the finite ordinary subcomplex $A_{n+1}^{(n)}\left(<H_{n+1}\right)$ of $A_{n+1}^{(n)}$. If $x \in A_{n+1}^{(n)}\left(\leqslant H_{n+1}\right)$ we have $G_{x} \subset H_{n+1}$. Since $f$ is isovariant it follows that

$$
f\left(A_{n+1}^{(n)}\left(\leqslant H_{n+1}\right)\right) \subset D^{m}(\rho)-B\left(H_{n+1}\right)
$$

and hence

$$
\operatorname{dist}\left(f\left(A_{n+1}^{(n)}\left(\leqslant H_{n+1}\right)\right), B\left(H_{n+1}\right)\right)=d_{n+1}>0 .
$$

Let $\delta_{n+1}>0$ be such that if $d(x, y)<\delta_{n+1}$, any $x, y \in X$, then

$$
\|f(x)-f(y)\|<\frac{1}{2} \min \left(d_{n+1}, \varepsilon\right) .
$$

Now let $\left(A_{n+1}^{(n)}\right)^{*}=C\left(X_{n}^{(n)}, X^{(n)}\right)^{*}$ be an equivariant subdivision of $A_{n+1}^{(n)}$ such that

$$
\operatorname{mesh}\left(\left(A_{n+1}^{(n)}\right)^{*}\right)<\delta_{n+1}
$$

Then define $X^{(n+1)}=X^{(n) *}$ to be the join extension of $A_{n+1}^{(n) *}=C\left(X_{n}^{(n)}, X^{(n) *}\right)$ to $X^{(n)}$. Then $X^{(n+1)}$ is an equivariant subdivision of $X^{(n)}$ such that $A_{n+1}^{(n+1)}=A_{n+1}^{(n) *}$, and also $X_{n}^{(n+1)}=X_{n}^{(n)}$. By the induction assumption we have $A_{i+1}^{(n)}=C\left(X_{i}^{(n)}, X^{(n)}\right)$, for $0 \leqslant i<n$. Since $X_{i}^{(n)} \subset X_{n}^{(n)}$, for $0<i<n$, it follows by the properties of the join extension from $C\left(X_{n}^{(n)}, X^{(n)}\right)^{*}$ to $X^{(n)}$ that we have for $0<i<n$

$$
A_{i+1}^{(n+1)}=A_{i+1}^{(n) *}=C\left(X_{i}^{(n)}, X^{(n)}\right)^{*}=C\left(X_{i}^{(n) *}, X^{(n) *}\right)=C\left(X_{i}^{(n+1)}, X^{(n+1)}\right) .
$$

This and the definition $A_{n+2}^{(n+1)}=C\left(X_{n+1}^{(n+1)}, X^{(n+1)}\right)$ shows that condition (a) is satisfied and $\operatorname{since} \operatorname{mesh}\left(A_{n+1}^{(n+1)}\right)=\operatorname{mesh}\left(A_{n+1}^{(n) *}\right)<\delta_{n+1}$ we also see that condition (b) is satisfied. This completes the inductive step. 
Now let $X^{\prime}=X^{(r)}$. Then we have

$$
\begin{gathered}
A_{i+1}^{\prime}=C\left(X_{i}^{\prime}, X^{\prime}\right), \quad 0 \leqslant i \leqslant r, \quad \text { and } \\
\operatorname{mesh}\left(A_{i}^{\prime}\right) \leqslant \operatorname{mesh}\left(A_{i}^{(i)}\right)<\delta_{i}, \quad 1 \leqslant i \leqslant r .
\end{gathered}
$$

Also $X^{\prime}=A_{1}^{\prime} \supset \cdots \supset A_{r}^{\prime} \supset A_{r+1}^{\prime}=\varnothing$, and the orbit types occurring in $A_{i}^{\prime}$, $1 \leqslant i \leqslant r$, are $\left(H_{i}\right), \ldots,\left(H_{r}\right)$. Now let $s$ be an arbitrary simplex of $X^{\prime}$. Let $k$, $1 \leqslant k \leqslant r$, be such that $\left(I_{0}(s)\right)=\left(H_{k}\right)$. Since $A_{k}^{\prime}=C\left(X_{k-1}^{\prime}, X^{\prime}\right)$ it then follows that $s$ is a simplex of $A_{k}^{\prime}$. Thus we have

$$
\operatorname{diam}(s) \leqslant \operatorname{mesh}\left(A_{k}^{\prime}\right)<\delta_{k}
$$

and hence

$$
\operatorname{diam}(f(s))<\frac{1}{2} \min \left(d_{k}, \varepsilon\right)
$$

where $d_{k}=\operatorname{dist}\left(f\left(A_{k}^{(k-1)}\left(\leqslant H_{k}\right)\right), B\left(H_{k}\right)\right)$. Now observe that since no point in $A_{k}^{(k-1)}$ has isotropy subgroup strictly greater than $H_{k}$ it follows that for any equivariant subdivision $A_{k}^{(k-1) *}$ of $A_{k}^{(k-1)}$ we have

$$
\left|A_{k}^{(k-1) *}\left(\leqslant H_{k}\right)\right| \subset\left|A_{k}^{(k-1)}\left(<H_{k}\right)\right| \text {. }
$$

Let $g \in G$ be such that $g I_{0}(s) g^{-1}=H_{k}$. Then $I_{0}(g s)=H_{k}$ and hence $g s$ is a simplex of $A_{k}^{\prime}\left(\leqslant H_{k}\right)$. Thus we have

$$
|g s| \subset\left|A_{k}^{\prime}\left(\leqslant H_{k}\right)\right| \subset\left|A_{k}^{(k-1)}\left(\leqslant H_{k}\right)\right| \text {. }
$$

Hence it follows that

$$
\begin{aligned}
d_{k} & \leqslant \operatorname{dist}\left(f(g s), B\left(H_{k}\right)\right)=\operatorname{dist}\left(f(s), g^{-1} B\left(H_{k}\right)\right) \\
& =\operatorname{dist}\left(f(s), B\left(g^{-1} H_{k} g\right)\right)=\operatorname{dist}\left(f(s), B\left(I_{0}(s)\right)\right) .
\end{aligned}
$$

Thus we have proved that

$$
\operatorname{diam}(f(s))<\frac{1}{2} \min \left(d_{s}, \varepsilon\right) .
$$

We now define $h: X^{\prime} \rightarrow D^{m}(\delta)$ as follows. If $s_{1}=\left\langle v_{0}, \ldots, v_{k}\right\rangle$ is a simplex of $Y_{1}^{\prime}$ define $h$ on the vertices of $s$ by $h\left(v_{i}\right)=f\left(v_{i}\right), i=0, \ldots, k$, and then define $h$ on all of $s_{1}$ by extending linearly. If $s_{0}$ is a simplex of $X^{\prime}$ such that $s_{0} \cap Y_{1}^{\prime}=\varnothing$ we define $h$ on $s$ by $h\left|s_{0}=f\right| s_{0}$. Now let $s$ be a simplex of $X^{\prime}-Y_{1}^{\prime}$ such that $s \cap Y_{1}^{\prime} \neq \varnothing$. Since $Y_{1}^{\prime}$ is full in $X^{\prime}$ we have, in a unique way, $s=s_{1} s_{0}$ where $s_{1}<Y_{1}^{\prime}$ and $s_{0} \cap Y_{1}^{\prime}=\varnothing$. Then define $h$ on $s$ by extending linearly the map $h$ already defined on $s_{1}$ and $s_{0}$. Since $f$ is a $G$-map this gives us a $G$-map $h$ : $X^{\prime} \rightarrow D^{m}(\delta)$, and $h \mid\left(Y_{1} \cup Y_{2}\right)^{\prime}$ is linear and $h\left|\left(Y_{2} \cup Y_{3}\right)^{\prime}=f\right|\left(Y_{2} \cup Y_{3}\right)^{\prime}$.

Let us now show that $h: X^{\prime} \rightarrow D^{m}(\delta)$ is isovariant. For this first observe that if $s=s_{1} s_{0}$, where $s_{1}<Y_{1}^{\prime}$ and $s_{0} \cap Y_{1}^{\prime}=\varnothing$, and $\left[a_{1}, b\right] \subset s$ is the line segment from a point $a_{1} \in s_{1}$ to any point $b \in s$, then $h$ is linear on $\left[a_{1}, b\right]$, i.e., we have

$$
h\left((1-t) a_{1}+t b\right)=(1-t) h\left(a_{1}\right)+t h(b), \quad a_{1} \in s_{1}, b \in s \text { and } 0 \leqslant t \leqslant 1 \text {. }
$$

(This is easily verified using the fact that $a_{1} \in s_{1}$ and that $h \mid s_{1}$ is linear. It is also at this point that we use the fact that we have the setup with subcomplexes $Y_{1}, Y_{2}$ and $Y_{3}$, where $Y_{1} \cap Y_{3}=\varnothing$, and not a setup of the type used in Lemma 2.1 with 
subcomplexes $Y$ and $Z$. Compare the construction of the map $h$ above with the construction of the map $h$ in the proof of Lemma 2.1.) We are now ready to prove that $h$ is isovariant. Let $s$ be an arbitrary $n$-simplex of $X^{\prime}$. We claim that if $x \in s$ then $G_{h(x)}=G_{x}$. This claim we prove by induction in $n$. If $n=0$, we have $s=\langle v\rangle$ a vertex. Since $h$ and $f$ agree on vertices and $f$ is isovariant we have $G_{h(v)}=G_{f(v)}=$ $G_{v}$. Now assume that we have proved our claim for the value $n-1$, where $n>1$. Let $s$ be any $n$-simplex of $X^{\prime}$ and $x \in s$. If $s \cap Y_{1}^{\prime}=\varnothing$ then $h|s=f| s$ and hence $G_{h(x)}=G_{f(x)}=G_{x}$. Assume next that $s \cap Y_{1}^{\prime} \neq \varnothing$. Then $s=s_{1} s_{0}$ where $s_{1}<Y_{1}^{\prime}$, $s_{1} \neq \varnothing$ and $s_{0} \cap Y_{1}^{\prime}=\varnothing$ (where $s_{0}=\varnothing$ is possible). Since $Y_{1}^{\prime}$ is strongly full in $X^{\prime}$ it follows that one of the vertices of $s_{1}$ has isotropy subgroup equal to $I_{0}(s)$. In fact it also follows that all the vertices of $s_{0}$ have isotropy subgroup equal to the principal, i.e., minimal, isotropy subgroup of $s$. Thus we can write $s=$ $\left\langle v_{0}, \ldots, v_{n}\right\rangle$ and $s_{1}=\left\langle v_{0}, \ldots, v_{k}\right\rangle$, where $0 \leqslant k \leqslant n$, with $G_{v_{0}}=I_{0}(s)$. Now if $x$ belongs to some $(n-1)$-dimensional face of $s$ then it follows by the induction assumption that $G_{h(x)}=G_{x}$. Thus we can assume that $x=\sum_{i=0}^{n} t_{i} v_{i}$, where $\sum_{i=0}^{n} t_{i}$ $=1$ and $t_{i}>0$, for all $i=0, \ldots, n$. Then we have $G_{x}=G_{v_{n}}$ the principal isotropy subgroup of $s$.

It follows from the way the map $h$ is constructed that we have $\operatorname{diam}(h(s))<$ $\operatorname{diam}(f(s))$. Thus, since $h\left(v_{0}\right)=f\left(v_{0}\right)$, we have

$$
\|h(y)-f(y)\| \leqslant \operatorname{diam}(h(s))+\operatorname{diam}(f(s)) \leqslant 2 \operatorname{diam}(f(s)),
$$

for any $y \in s$. Hence it follows from (*) that

$$
\|h(y)-f(y)\|<\operatorname{dist}\left(f(s), B\left(I_{0}(s)\right)\right)
$$

for every $y \in s$. Hence we have $h(y) \notin B\left(I_{0}(S)\right)$, for every $y \in S$. Therefore we have $G_{h(y)} \subset I_{0}(s)$ for every $y \in s$ and in particular

$$
G_{h(x)} \subset I_{0}(s)=G_{v_{0}} \text {. }
$$

Now let

$$
b=\left(1-\frac{1}{1-t_{0}}\right) v_{0}+\frac{1}{1-t_{0}} x=\sum_{i=1}^{n} \frac{t_{i}}{1-t_{0}} v_{i}
$$

Since $b$ lies in an $(n-1)$-dimensional face of $s$ we have by the induction assumption that $G_{h(b)}=G_{b}=G_{v_{n}}$. Since

$$
x=t_{0} v_{0}+\left(1-t_{0}\right) b
$$

and $v_{0} \in s_{1}$, we have by (\#) that

$$
h(x)=t_{0} h\left(v_{0}\right)+\left(1-t_{0}\right) h(b)
$$

that is

$$
h(b)=\frac{1}{1-t_{0}}\left(h(x)-t_{0} h\left(v_{0}\right)\right) .
$$

Since $G_{h(x)} \subset G_{v_{0}}$ this shows that the point $h(b)$ is fixed under $G_{h(x)}$, i.e.,

$$
G_{h(x)} \subset G_{h(b)} \text {. }
$$

Since $G_{h(b)}=G_{v_{n}}=G_{x}$ we get $G_{h(x)} \subset G_{x}$, and hence $G_{h(x)}=G_{x}$ since $h$ is a $G$-map. This completes the induction and proofs that $h$ is isovariant. 
By (**) and (*) we have that $\|h(y)-f(y)\|<\varepsilon$ for any $y \in X$. Since also $h|| Y_{2} \cup Y_{3}|=f|\left|Y_{2} \cup Y_{3}\right|$ it follows that $H: X \times I \rightarrow D^{m}(\delta)$, defined by $H(y, \tau)$ $=(1-\tau) f(y)+\tau h(y)$, is an equivariant $\varepsilon$-homotopy rel $\left|Y_{2} \cup Y_{3}\right|$ from $f$ to $h$.

Proposition 2.4. Let $Y_{1}, Y_{2}$ and $Y_{3}$ be as in Lemma 2.3, and let $f: X \rightarrow D^{m}(\delta)$, be an isovariant map such that $f \mid: Y_{2} \rightarrow D^{m}(\delta)_{t}$ is simplicial. Let $\varepsilon>0$ be given. Then there exist equivariant subdivisions $X^{*}$ and $D^{m}(\delta)_{t}^{*}$ of $X$ and $D^{m}(\delta)_{t}$, respectively, and an isovariant map $h: X^{*} \rightarrow D^{m}(\delta)_{t}^{*}$ such that $h \mid:\left(Y_{1} \cup Y_{2}\right)^{*} \rightarrow D^{m}(\delta)_{t}^{*}$ is simplicial and $h$ is equivariantly $\varepsilon$-homotopic rel $\left|Y_{2} \cup Y_{3}\right|$ to $f$.

Proof. This follows easily from Lemma 2.3 using Lemma 1.5.

EXAMPLE. Let $G=\mathbf{Z}_{2}$ and $X=I \times \mathbf{Z}_{2}$, where the unit interval $I$ has vertices 0 and 1 , and $\mathbf{Z}_{2}$ acts on $X$ by trivial action on $I$ and by multiplication on $\mathbf{Z}_{2}$. Let $\mathbf{R}^{2}(\tau)=\mathbf{R} \oplus \mathbf{R}( \pm)$, where $\mathbf{Z}_{2}$ acts trivially on $\mathbf{R}$ and acts by $T x=-x$ on $\mathbf{R}( \pm)$. Define a linear $\mathbf{Z}_{2}$-map $f: X \rightarrow \mathbf{R}^{2}(\tau)$ by defining $f(0, e)=(1,1)$ and $f(1, e)=$ $(3,-1)$ and extending linearly and equivariantly. Then $f\left(\frac{1}{2}, e\right)=(2,0)$ and $f\left(\frac{1}{2}, T\right)$ $=(2,0)$, and hence $f$ is not equivariantly nondegenerate $(f$ is nondegenerate in the ordinary sense). Moreover it is impossible to alter $f$, by a small change, into an equivariantly nondegenerate map. Observe that $\operatorname{dim} X^{G}=-1 \leqslant \operatorname{dim} \mathbf{R}^{2}(\rho)^{G}=1$ and $\operatorname{dim} X^{\{e\}}=1 \leqslant \operatorname{dim} \mathbf{R}^{2}(\rho)^{\{e\}}=2$. Now consider $f$ as a $\mathbf{Z}_{2}$-map $f: X \rightarrow \mathbf{R}^{2}(\tau) \oplus$ $\mathbf{R}( \pm)=\mathbf{R}^{3}(\rho)$. Let $\varepsilon>0$ and define a linear $\mathbf{Z}_{2}$-map $h: X \rightarrow \mathbf{R}^{3}(\rho)$ by defining $h(0, e)=(1,1, \varepsilon)$ and $h(1, e)=(3,-1,0)$ and extending linearly and equivariantly. Then $h$ is equivariantly nondegenerate and an $\varepsilon$-approximation to $f$. Observe that $\operatorname{dim} X^{\{e\}}=1$ and $\operatorname{dim} \mathbf{R}^{3}(\rho)^{\{e\}}-\operatorname{dim} \mathbf{R}^{3}(\rho)^{>\{e\}}-1=3-1-1=1$.

This example leads us to the next lemma. It is the version in parentheses that is of primary interest to us. Observe that since $\operatorname{dim} \varnothing=-1$ the dimension assumption in Lemma 2.5 in case $G=\{e\}$, i.e., in the ordinary nonequivariant case, is the correct one; $\operatorname{dim}(Y-Z) \leqslant \operatorname{dim} D^{m}=m$.

REMARK. Observe that if a subgroup $H$ of $G$ does not occur as an isotropy subgroup of some point in $Y$, then $Y^{H}-H^{>H}=Y_{H}=\varnothing$. It follows that in Lemma 2.5 below the expression "for every subgroup $H$ of $G$ " could as well be changed in the following way. Let $H_{1}, \ldots, H_{t}$ be subgroups of $G$ forming a complete set of representatives for the orbit types occurring in $Y$. Then we need only assume that the dimension assumption in Lemma 2.5 holds for the subgroups $H_{1}, \ldots, H_{t}$. We have chosen the formulation "for every subgroup $H$ of $G$ " for sheer convenience. This same remark also concerns the formulation of many other results in this paper.

LEMMA 2.5. Let $Y$ and $Z$ be equivariant subcomplexes of $X$ such that $Y \cap Z$ is strongly full in $Y$. Let $f: X \rightarrow D^{m}(\rho)$ be a linear $G$-map such that $f \mid: Y \cap Z \rightarrow$ $D^{m}(\rho)$ is isovariant (isovariant and nondegenerate). Assume that

$$
\operatorname{dim}\left(Y^{H}-\left(Y^{>H} \cup Z\right)\right) \leqslant \operatorname{dim} D^{m}(\rho)^{H}-\operatorname{dim} D^{m}(\rho)^{>H}-1
$$

for every subgroup $H$ of $G$. Then, given $\varepsilon>0, f$ is equivariantly $\varepsilon$-homotopic rel $|Z|$ to a linear G-map $h: X \rightarrow D^{m}(\rho)$ such that $h \mid: Y \rightarrow D^{m}(\rho)$ is isovariant (isovariant and

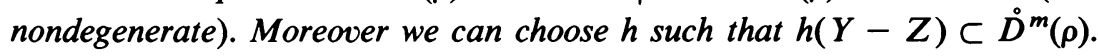


Proof. Let $H_{1}, \ldots, H_{s}$ be subgroups of $G$ forming a complete set of representatives for the orbit types occurring in $X$, ordered in such a way that $\left(H_{i}\right)>\left(H_{j}\right)$ implies $i<j$. Let us denote $Y_{0}=Y \cap Z$ and

$$
Y_{n}=\bigcup_{i=1}^{n} G Y^{H_{i}} \cup(Y \cap Z), \quad n=1, \ldots, s .
$$

Since $Y \cap Z$ is strongly full in $Y$ it follows by Lemma 1.4, that $Y_{n-1}$ is full in $Y$ and hence also in $Y_{n}, n=1, \ldots, s$. Observe that we have

$$
Y_{n}-Y_{n-1}=G\left(Y^{H_{n}}-\left(Y^{>H_{n}} \cup Z\right)\right), \quad n=1, \ldots, s .
$$

We shall construct linear $G$-maps

$$
h_{n}: X \rightarrow D^{m}(\rho), \quad n=0, \ldots, s,
$$

such that

1. $h_{n}\left|Y_{n-1}=h_{n-1}\right| Y_{n-1}, n \geqslant 1$,

2. $\left\|h_{n}(x)-f(x)\right\| \leqslant \varepsilon$, for every $x \in X$,

3. $h_{n} \mid: Y_{n} \rightarrow D^{m}(\rho)$ is isovariant (isovariant and nondegenerate),

4. $h_{n}\left(Y_{n}-Z\right) \subset D^{m}(\rho)$,

5. $h_{n}|Z=f| Z$.

Let $h_{0}=f$ and assume inductively that $h_{0}, \ldots, h_{n-1}$, where $n \geqslant 1$, have been defined and satisfy the above conditions. We now construct $h_{n}$ as follows. Let $G v_{1}, \ldots, G v_{p}, G v_{p+1}, \ldots, G v_{q}$ be the equivariant vertices of $Y_{n}$, where $G v_{1}, \ldots, G v_{p}$ are the ones in $Y_{n-1}$ and $G v_{p+1}, \ldots, G v_{q}$ are the ones in $Y_{n}-Y_{n-1}$, and where we moreover have chosen $v_{p+1}, \ldots, v_{q} \in Y^{H_{n}}-\left(Y^{>H_{n}} \cup Z\right)$. Thus we have $G_{v_{i}}=H_{n}$, for $i=p+1, \ldots, q$. Let us denote $h_{n-1}\left(v_{i}\right)=y_{i}, i=1, \ldots, p$. Inductively we now construct points

$$
y_{p+1}, \ldots, y_{q} \in D^{m}(\rho)^{H_{n}}
$$

such that the following conditions will be satisfied. We denote below

$$
k=\operatorname{dim}\left(Y^{H_{n}}-\left(Y^{>H_{n}} \cup Z\right)\right) \text {. }
$$

(i) Denoting $S=\left\{y_{1}, \ldots, y_{q}\right\}$ we have that

$$
y_{j} \notin\left\langle\left\langle\mathbf{R}^{m}(\rho)^{H}, g_{1} z_{1}, \ldots, g_{l} z_{l}\right\rangle\right\rangle, \quad p+1<j<q,
$$

for every subgroup $H$ of $G$ such that $H_{n} \varsubsetneqq H$, and any $z_{1}, \ldots, z_{l} \in S-\left\{y_{j}\right\}$ and $g_{1}, \ldots, g_{l} \in G$ with $l \leqslant k$. (Recall that $\left\langle\left\langle\mathbf{R}^{m}(\rho)^{H}, g_{1} z_{1}, \ldots, g_{l} z_{l}\right\rangle\right\rangle$ denotes the affine subspace of $\mathbf{R}^{m}(\rho)$ generated by the set $\mathbf{R}^{m}(\rho)^{H} \cup\left\{g_{1} z_{1}, \ldots, g_{l} z_{l}\right\}$.)

(ii)

$$
d\left(y_{j}, f\left(v_{j}\right)\right)<\varepsilon, \quad p+1 \leqslant j \leqslant q .
$$

This is possible, since for $H_{n} \varsubsetneqq H$ we have

$$
\begin{array}{r}
\operatorname{dim}\left\langle\left\langle\mathbf{R}^{m}(\rho)^{H}, g_{1} z_{1}, \ldots, g_{l} z_{l}\right\rangle\right\rangle \leqslant \operatorname{dim} \mathbf{R}^{m}(\rho)^{>H_{n}}+l \\
\leqslant \operatorname{dim} D^{m}(\rho)^{>H_{n}}+k \leqslant \operatorname{dim} D^{m}(\rho)^{H_{n}}-1,
\end{array}
$$

and since $G$ is finite.

Now let $G w_{1}, \ldots, G w_{r}$ denote the equivariant vertices of $X-Y_{n}$ and construct a linear $G$-map $h_{n}: X \rightarrow D^{m}(\rho)$ by defining it on the equivariant vertices of $X$ in the 
following way:

(a) $h_{n}\left|G v_{i}=h_{n-1}\right| G v_{i}, 1 \leqslant i \leqslant p$.

(b) $h_{n} \mid: G v_{i} \rightarrow D^{m}(\rho)$ is defined by $h_{n}\left(g v_{i}\right)=g y_{i}$, for all $g \in G$ and $p+1 \leqslant i \leqslant$ $q$,

(c) $h_{n}\left|G w_{j}=f\right| G w_{j}, 1 \leqslant j \leqslant r$,

and then extending linearly to the equivariant simplexes of $X$. (Observe that the definition given in (b) gives a well-defined $G$-map since $G_{v_{i}}=H_{n}$ and $y_{i} \in$ $D^{m}(\rho)^{H_{n}}$, for $p+1 \leqslant i \leqslant q$.)

It follows directly from the above construction that $h_{n}$ satisfies conditions 1, 2, 4 and 5. We claim that $h_{n} \mid: Y_{n} \rightarrow D^{m}(\rho)$ is isovariant (isovariant and nondegenerate). We first prove that $h_{n} \mid Y_{n}$ is isovariant. Let $A$ be an equivariant simplex in $Y_{n}$. We shall show that $h_{n} \mid: A \rightarrow D^{m}(\rho)$ is isovariant. If $A \subset Y_{n-1}$ this follows from the induction hypothesis. Thus we can assume that $A$ is an equivariant simplex of $Y_{n}-Y_{n-1}$ and hence we have $\operatorname{dim} A \leqslant k$. Since $Y_{n-1}$ is full in $Y_{n}$ at least one of the equivariant vertices of $A$ lies in $Y_{n}-Y_{n-1}$. Let $A=G s$ where $s$ is an ordinary simplex and we have chosen $s \subset Y^{H_{n}}$. Let $a_{0}, \ldots, a_{e}, a_{e+1}, \ldots, a_{d}$ be the vertices of $s$, where $a_{e+1}, \ldots, a_{d}$ denote the ones belonging to $Y^{H_{n}}-\left(Y^{>H_{n}} \cup Z\right)$, and we have $-1 \leqslant e<d$. Now we have $a_{i}=g_{i} v_{j_{i}}$, where $g_{i} \in G, i=0, \ldots, d$, and where $v_{j_{i}}$ and $v_{j_{i}}$, belong to different equivariant vertices, i.e., different orbits, if $i \neq i^{\prime}$. Moreover $e+1 \leqslant i \leqslant d$ implies that $p+1 \leqslant j_{i} \leqslant q$ and that $g \in N\left(H_{n}\right)$.

Let $x \in s$, we claim that $G_{h_{n}(x)}=G_{x}$. If $x$ belongs to a face of $s$ which lies in $Y_{n-1}$ this follows from the induction hypothesis. Thus we can assume, since $Y_{n-1}$ is full in $Y_{n}$, that $x=\sum_{i=0}^{d} t_{i} a_{i}$, with $\sum_{i=0}^{d} t_{i}=1, t_{i} \geqslant 0$, and at least one $t_{i^{\prime}} \neq 0$, where $e+1 \leqslant i^{\prime} \leqslant d$, say $t_{d} \neq 0$. Denote $G_{h_{n}(x)}=K$, thus we have $H_{n}=G_{x} \subset K$. Since $h_{n}(x)=\sum_{i=0}^{d} t_{i} g_{i} y_{j_{i}} \in \mathbf{R}^{m}(\rho)^{K}$ and $t_{d} \neq 0$ it follows that

$$
y_{j_{d}} \in\left\langle\left\langle\mathbf{R}^{m}(\rho)^{K^{\prime}}, g_{0}^{\prime} y_{j_{0}}, \ldots, g_{d-1}^{\prime} y_{j_{d-1}}\right\rangle\right\rangle \text {, }
$$

where we have denoted $K^{\prime}=g_{d}^{-1} K g_{d}$ and $g_{i}^{\prime}=g_{d}^{-1} g_{i}, i=0, \ldots, d-1$. If $H_{n} \varsubsetneqq K$ then, since $g_{d} \in N\left(H_{n}\right)$, we also have $H_{n} \varsubsetneqq K^{\prime}$. Since $d \leqslant k$ and $y_{j_{i}} \in S-\left\{y_{j_{d}}\right\}$, $i=0, \ldots, d-1$, and $p+1 \leqslant j_{d} \leqslant q$ we see that (*) is in contradiction with (i). Thus $H_{n}=K$, that is, $G_{x}=G_{h(x)}$. It now follows that $h_{n} \mid A$ is isovariant and hence that $h_{n} \mid: Y_{n} \rightarrow D^{m}(\rho)$ is isovariant.

It now remains to prove, assuming $f \mid Y \cap Z$ to be nondegenerate, that $h_{n} \mid Y_{n}$ is nondegenerate. From the inductive assumption that $h_{i} \mid Y_{i}, i=0, \ldots, n-1$, are nondegenerate and condition (i) it follows that if $s=\left\langle a_{0}, \ldots, a_{d}\right\rangle$ is a simplex of $Y_{n}$ then the points $h_{n}\left(a_{0}\right), \ldots, h_{n}\left(a_{d}\right)$ are affinely independent and hence $h_{n} \mid s$ is an imbedding.

Now $h=h_{s}: X \rightarrow D^{m}(\rho)$ and the (not linear) homotopy $H: X \times I \rightarrow D^{m}(\rho)$, defined by $H(x, t)=(1-t) f(x)+t h(x)$, satisfy the conclusions of the lemma.

Proposition 2.6. Let $Y_{1}, Y_{2}$ and $Y_{3}$ be as in Lemma 2.3, and let $f: X \rightarrow D^{m}(\rho)_{t}$, be a simplicial G-map such that $f \mid: Y_{2} \rightarrow D^{m}(\rho)_{t}$ is isovariant (equivariantly nondegenerate). Assume that

$$
\operatorname{dim}\left(Y_{1}^{H}-\left(Y_{1}^{>H} \cup Y_{2}\right)\right) \leqslant \operatorname{dim} D^{m}(\rho)^{H}-\operatorname{dim} D^{m}(\rho)^{>H}-1
$$


for every subgroup $H$ of $G$. Let $\varepsilon>0$ be given. Then there exist equivariant subdivisions $X^{*}$ and $D^{m}(\rho)_{t}^{*}$ of $X$ and $D^{m}(\rho)_{t}$, respectively, and a simplicial G-map $h$ : $X^{*} \rightarrow D^{m}(\rho)_{t}^{*}$ such that $h \mid:\left(Y_{1} \cup Y_{2}\right)^{*} \rightarrow D^{m}(\rho)_{t}^{*}$ is isovariant (equivariantly nondegenerate) and such that $h$ is equivariantly $\varepsilon$-homotopic rel| $Y_{2} \cup Y_{3} \mid$ to f. Moreover we can choose $h$ such that $h\left(Y_{1}-Y_{2}\right) \subset D^{m}(\rho)$.

Proof. Let $Y=Y_{1} \cup Y_{2}$ and $Z=Y_{2} \cup Y_{3}$. Since $Y_{1} \cap Y_{3}=\varnothing$ we then have $Y \cap Z=Y_{2}$. Also observe that $Y-Z=Y_{1}-Y_{2}$ and $Y^{H}-\left(Y^{>H} \cup Z\right)=Y_{1}^{H}$ - $\left(Y_{1}^{>H} \cup Y_{2}\right)$ for any subgroup $H$ of $G$. Let $X^{\prime}$ be an equivariant subdivision of $X$ such that $Y_{2}^{\prime}=(Y \cap Z)^{\prime}$ is strongly full in $Y^{\prime}$. Applying Lemma 2.5 to the linear $G$-map $f: X^{\prime} \rightarrow D^{m}(\rho)$ we get a linear $G$-map $h: X^{\prime} \rightarrow D^{m}(\rho)$ such that $h \mid Y^{\prime}$ is isovariant (isovariant and nondegenerate) and $h$ is equivariantly $\varepsilon$-homotopic $\operatorname{rel}|Z|$ to $f$ and $h(Y-Z) \subset D^{m}(\rho)$. By Lemma 1.5 there exist an equivariant subdivision $X^{\prime \prime}$ of $X^{\prime}$ and an equivariant triangulation $D^{m}(\rho)_{t_{0}}$ of $D^{m}(\rho)$ such that $h: X^{\prime \prime} \rightarrow$ $D^{m}(\rho)_{t_{0}}$ is simplicial. Let $D^{m}(\rho)_{t_{0}}^{*}$ be a common equivariant subdivision of $D^{m}(\rho)_{t}$ and $D^{m}(\rho)_{t_{0}}$. Since $h: X^{\prime \prime} \rightarrow D^{m}(\rho)_{t_{0}}$ is a simplicial $G$-map and $D^{m}(\rho)^{*}$ is an equivariant subdivision of $D^{m}(\rho)_{t_{0}}$ there exists an equivariant subdivision $X^{*}$ of $X^{\prime \prime}$ such that $h: X^{*} \rightarrow D^{m}(\rho)_{t}^{*}$ is a simplicial $G$-map. In the case when $h \mid Y^{\prime}$ is isovariant and nondegenerate we have by Lemma 1.7 that $h \mid: Y^{*} \rightarrow D^{m}(\rho)_{t}^{*}$ is equivariantly nondegenerate.

Lemma 2.7. Let $f: X \rightarrow \mathbf{R}^{m}(\rho)$ be a linear isovariant map. Then there exists $\delta>0$ such that any linear G-map $h: X \rightarrow \mathbf{R}^{m}(\rho)$ satisfying $\|f(x)-h(x)\|<\delta$, for every $x \in X$, is isovariant and moreover isovariantly $\delta$-homotopic to $f$.

Proof. Let $s$ be a simplex of $X$ and denote the maximal isotropy subgroup of $s$ by $I_{0}(s)$. Since $f$ is isovariant the isotropy subgroup at any point in $f(s)$ is a subgroup of $I_{0}(s)$. For any subgroup $H_{0}$ of $G$ we denote

$$
J\left(H_{0}\right)=\left\{H \text { is a subgroup of } G \mid H \nsubseteq H_{0}\right\} .
$$

Now for any simplex $s$ of $X$ we set

$$
B_{s}=\bigcup_{H \in J\left(I_{0}(s)\right)} \mathbf{R}^{m}(\rho)^{H} .
$$

Thus $f(s) \cap B_{s}=\varnothing$, and since $f(s)$ is compact and $B_{s}$ is closed it follows that $d_{s}=\operatorname{dist}\left(f(s), B_{s}\right)>0$. Now set

$$
\delta=\min \left\{d_{s} \mid s \text { is a simplex of } X\right\} .
$$

We shall show that if $h: X \rightarrow \mathbf{R}^{m}(\rho)$ is a linear $G$-map such that $\|f(x)-h(x)\|<$ $\delta$, for every $x \in X$, then $h$ is isovariant. Let $s$ be an $n$-simplex of $X$. We claim that if $x \in s$ then $G_{h(x)}=G_{x}$. This we prove by induction in $n$. If $n=0$, then $s=\left\langle v_{0}\right\rangle$ a vertex. Since $h$ is a $G$-map we have $G_{v_{0}} \subset G_{h\left(v_{0}\right)}$. Since $\left\|f\left(v_{0}\right)-h\left(v_{0}\right)\right\|<\delta \leqslant d_{s}$, we have $h\left(v_{0}\right) \notin B_{s}$ and hence $G_{h\left(v_{0}\right)} \subset G_{v_{0}}$. Thus $G_{h\left(v_{0}\right)}=G_{v_{0}}$. Now assume that we have proved our claim for the value $n-1$, where $n \geqslant 1$. Let $s=\left\langle v_{0}, \ldots, v_{n}\right\rangle$ be an $n$-simplex of $X$ and $x \in s$. Here the vertices of $s$ are ordered such that $G_{v_{0}}$ is the maximal isotropy subgroup of $s$ and $G_{v_{n}}$ is the principal isotropy subgroup of $s$, i.e., the minimal isotropy subgroup of $s$. If $x$ belongs to some $(n-1)$-dimensional face of $s$ then it follows by the induction assumption that $G_{h(x)}=G_{x}$. Thus we can 
assume that $x=\sum_{i=0}^{n} t_{i} v_{i}$, where $\sum_{i=0}^{n} t_{i}=1, t_{i} \geqslant 0$ and $t_{i} \neq 0, i=0, \ldots, n$. Then we have $G_{x}=G_{v_{n}}$. Since $\|f(x)-h(x)\|<\delta \leqslant d_{s}$, we must have $G_{h(x)} \subset G_{v_{0}}$. Hence the point

$$
w=\left(1-\frac{1}{1-t_{0}}\right) h\left(v_{0}\right)+\frac{1}{1-t_{0}} h(x)=\sum_{i=1}^{n} \frac{t_{i}}{1-t_{0}} h\left(v_{i}\right)
$$

is fixed under the subgroup $G_{h(x)}$, that is, $G_{h(x)} \subset G_{w}$. But we have $w=h(y)$, where the point $y=\sum_{i=1}^{n} t_{i} v_{i} /\left(1-t_{0}\right)$ belongs to an $(n-1)$-dimensional face of $s$. Moreover $G_{y}=G_{v_{n}}$. By the induction assumption we have $G_{w}=G_{h(y)}=G_{y}$, and hence $G_{h(x)} \subset G_{v_{n}}$. But since $h$ is a $G$-map we have $G_{v_{n}}=G_{x} \subset G_{h(x)}$, and hence $G_{h(x)}=G_{x}$. This completes the induction step and shows that $h$ is isovariant.

We get a well-defined $G$-homotopy $F: X \times I \rightarrow \mathbf{R}^{m}(\rho)$ by defining $f(x, t)=$ $(1-t) f(x)+t h(x)$; and since for each fixed $t$ the map $F_{t}: X \rightarrow \mathbf{R}^{m}(\rho)$, defined by $F_{t}(x)=F(x, t)$ is a linear $G$-map satisfying $\left\|f(x)-F_{t}(x)\right\|<\delta$, for every $x \in X$, it follows that $F_{t}$ is isovariant. Hence $F$ is an isovariant $\delta$-homotopy from $f$ to $h$.

LEMMA 2.8. Let $Y$ and $Z$ be equivariant subcomplexes of $X$ such that $Y \cap Z$ is strongly full in $Y$. Let $f: X \rightarrow D^{m}(\rho)$ be a linear isovariant map such that $f \mid$ : $Y \cap Z \rightarrow D^{m}(\rho)$ is nondegenerate. Assume that

$$
\operatorname{dim}\left(Y^{H}-\left(Y^{>H} \cup Z\right)\right) \leqslant \operatorname{dim} D^{m}(\rho)^{H}
$$

for every subgroup $H$ of $G$. Then, given $\varepsilon>0, f$ is isovariantly $\varepsilon$-homotopic rel| $Z \mid$ to a linear isovariant map $h: X \rightarrow D^{m}(\rho)$ such that $h \mid: Y \rightarrow D^{m}(\rho)$ is nondegenerate. Moreover we can choose $h$ such that $h(Y-Z) \subset D^{m}(\rho)$.

Proof. The proof is very similar to that of Lemma 2.5. Let the notation be the same as in the proof of Lemma 2.5. First observe that using Lemma 2.7 we can assume that $\varepsilon>0$ is such that if $h^{\prime}: X \rightarrow D^{m}(\rho)$ is any linear $G$-map which is an $\varepsilon$-approximation to $f$, then $h^{\prime}$ is isovariant and moreover the homotopy $H: X \times I$ $\rightarrow D^{m}(\rho)$, defined by $H(x, t)=(1-t) f(x)+t h^{\prime}(x)$, is isovariant. Then proceed as in the proof of Lemma 2.5 , but this time, in constructing the linear $G$-map $h_{n}$ : $X \rightarrow D^{m}(\rho)$, choose the points $y_{p+1}, \ldots, y_{q} \in D^{m}(\rho)^{H_{n}}$ such that

$$
y_{j} \notin\left\langle\left\langle g_{1} z_{1}, \ldots, g_{l} z_{l}\right\rangle\right\rangle, \quad p+1<j \leqslant q,
$$

for every subgroup $H$ of $G$ such that $H_{n} \varsubsetneqq H$, any $k$ or less points $z_{1}, \ldots, z_{l} \in S$ $-\left\{y_{j}\right\}, l \leqslant k$, and any $g_{1}, \ldots, g_{l} \in G$, and also such that $d\left(y_{j}, f\left(v_{j}\right)\right)<\varepsilon, p+1 \leqslant$ $j \leqslant q$. This is possible since

$$
\operatorname{dim}\left\langle\left\langle g_{1} z_{1}, \ldots, g_{l} z_{l}\right\rangle\right\rangle\left\langle l-1 \leqslant k-1 \leqslant \operatorname{dim} D^{m}(\rho)^{H_{n}}-1 .\right.
$$

It then follows that the linear $G$-map $h_{n}: X \rightarrow D^{m}(\rho)$ is such that $h_{n} \mid: Y_{n} \rightarrow D^{m}(\rho)$ is nondegenerate and $h_{n}$ is an $\varepsilon$-approximation to $f$ and hence also that $h_{n}: X \rightarrow$ $D^{m}(\rho)$ is isovariant.

Proposition 2.9. Let $Y_{1}, Y_{2}$ and $Y_{3}$ be as in Lemma 2.3, and let $f: X \rightarrow D^{m}(\rho)$, be an isovariant simplicial map such that $f \mid: Y_{2} \rightarrow D^{m}(\rho)_{t}$ is equivariantly nondegenerate. Assume that

$$
\operatorname{dim}\left(Y_{1}^{H}-\left(Y_{1}^{>H} \cup Y_{2}\right)\right) \leqslant \operatorname{dim} D^{m}(\rho)^{H}
$$


for every subgroup $H$ of $G$. Let $\varepsilon>0$ be given. Then there exist equivariant subdivisions $X^{*}$ and $D^{m}(\rho)_{t}^{*}$ of $X$ and $D^{m}(\rho)_{t}$, respectively, and an isovariant simplicial map $h: X^{*} \rightarrow D^{m}(\rho)_{t}^{*}$ such that $h \mid:\left(Y_{1} \cup Y_{2}\right)^{*} \rightarrow D^{m}(\rho)_{t}^{*}$ is equivariantly nondegenerate and $h$ isovariantly $\varepsilon$-homotopic rel| $\left|Y_{2} \cup Y_{3}\right|$ to $f$. Moreover we can choose $h$ such that $h\left(Y_{1}-Y_{2}\right) \subset \stackrel{D^{m}}{ }(\rho)$.

Proof. This follows from Lemma 2.8 in exactly the same way as Proposition 2.6 followed from Lemma 2.5.

Let $M$ be an equivariant p.l. manifold, $R_{0} \subset M$ an equivariant subpolyhedron of $M$ and $\varepsilon: M \rightarrow \mathbf{R}$ an invariant positive function. Then an equivariant p.l. $\varepsilon$-isotopy of $M$ rel $R_{0}$ is a level-preserving p.l. $G$-homeomorphism $\Phi: M \times I \rightarrow M \times I$ such that $\Phi(x, 0)=(x, 0)$ for every $x \in M$ and $\Phi(y, t)=(y, t)$ for every $(y, t) \in R_{0} \times$ $I$ and $p_{2} \Phi: M \times I \rightarrow M$ is an $\varepsilon$-homotopy.

Given such a $\Phi$ we denote by $\Phi_{t}$ the p.l. $G$-homeomorphism $\Phi_{t}: M \rightarrow M$ defined by $\Phi_{t}(x)=p r_{2} \Phi(x, t)$. If $\Phi_{1}=h$ we also sometimes say that $\Phi$ is an equivariant ambient p.l. $\varepsilon$-isotopy of $M$ rel $R_{0}$ from id to $h$. Lemma 2.10 below is a straightforward equivariant version of the "Alexander trick".

LEMMA 2.10. Let $h: D^{m}(\tau) \rightarrow D^{m}(\tau)$ be a p.l. G-homeomorphism such that $h(x)=x$ for every $x \in \partial D^{m}(\tau)$, and moreover $\|h(x)-x\|<\varepsilon$ for all $x \in D^{m}(\tau)$, where $\varepsilon>0$. Then there exists an equivariant ambient p.l. $\varepsilon$-isotopy of $D^{m}(\tau)$ rel $\partial D^{m}(\tau)$ from id to $h$.

Proof. The proof is the same as in the ordinary case.

LemMA 2.11. Let $E$ denote a triangulation of $D^{m}(\rho)$, as an equivariant simplicial complex. Let $G v_{i} \in \stackrel{\circ}{E}, i=1, \ldots, n$, be some equivariant vertices of $D$ and assume that $K$ is an equivariant subcomplex of $E$ such that $K \cap \stackrel{\operatorname{S}}{\mathrm{t}}\left(v_{i}, E\right)=\varnothing, i=$ $1, \ldots, n$. Let

$$
w_{i} \in \stackrel{\circ}{\operatorname{St}}\left(v_{i}, E\right)^{G_{v_{i}}}
$$

such that $\left\|w_{i}-v_{i}\right\|<\varepsilon / n, i=1, \ldots, n$, and let $h: E \rightarrow D^{m}(\rho)$ be the linear G-map defined on the vertices by $h\left(g v_{i}\right)=g w_{i}, i=1, \ldots, n$, and $h(v)=v$ for every vertex $v \notin \cup \cup_{i=1}^{n} G v_{i}$, and extended linearly to the simplexes. Then $h: D^{m}(\rho) \rightarrow D^{m}(\rho)$ is a $G$-homeomorphism and there exists an equivariant p.l. $\varepsilon$-isotopy of $D^{m}(\rho)$ $\operatorname{rel}\left(K \cup \partial D^{m}(\rho)\right)$ from id to $h$.

Proof. It is easily seen that for each $i, 1 \leqslant i \leqslant n$, the linear $G$-map $h_{i}: E \rightarrow$ $D^{m}(\rho)$ defined on the vertices by $h_{i}\left(g v_{i}\right)=g w_{i}$ and $h(v)=v$ for all $v \notin G v_{i}$, and extended linearly to the simplexes, is a well-defined $G$-homeomorphism. We have $h_{i} \mid\left(E-\stackrel{\operatorname{St}}{ }\left(G v_{i}, E\right)\right)=$ id. Let $Q_{i}$ denote the $G_{v_{i}}$-equivariant subpolyhedron of $D^{m}(\rho)$ determined by $\operatorname{St}\left(v_{i}, E\right)$. Then $Q_{i}$ is p.l. $G_{v_{i}}$-homeomorphic to some $D^{m}(\tau)$, where $\tau_{i}: G_{v_{i}} \rightarrow O(m)$. Since $h_{i} \mid: Q_{i} \rightarrow Q_{i}$ is a p.l. $G_{v_{i}}$-homeomorphism such that $h_{i}(x)=x$ for $x \in \partial Q_{i}$ and $\left\|h_{i}(x)-x\right\|<\varepsilon / n$ for every $x \in Q_{i}$, it follows by Lemma 2.10 that there exists a $G_{v_{i}}$-equivariant ambient p.l. $(\varepsilon / n)$-isotopy $\Phi^{(i)}$ of $Q_{i}$ rel $\partial Q_{i}$ from id to $h_{i} \mid Q_{i}$. This gives us a $G$-equivariant ambient p.l. $(\varepsilon / n)$-isotopy $\tilde{\Phi}^{(i)}$ of $G Q_{i}$ rel $G\left(\partial Q_{i}\right)$ from id to $h_{i} \mid G Q_{i}$. Extending $\tilde{\Phi}^{(i)}$ by the identity map on 
$\left(D^{m}(\rho)-G Q_{i}\right) \times I$ we get a $G$-equivariant ambient p.l. $(\varepsilon / n)$-isotopy $\Phi^{(i)}$ of $D^{m}(\rho)$ $\operatorname{rel}\left(D^{m}(\rho)-G \dot{Q}_{i}\right)$ and hence $\operatorname{rel}\left(K \cup \partial D^{m}(\rho)\right)$ from id to $h_{i}$. Since $h=$ $h_{n} \circ \ldots \circ h_{1}$ we have that $\Phi=\Phi^{(n)} \circ \ldots \circ \Phi^{(1)}$ is a required isotopy.

Definition. Let $R$ and $V_{1}, \ldots, V_{r}$ be equivariant subpolyhedra of the equivariant p.l. manifold $M$, and $R_{0}$ an equivariant subpolyhedron of $R$. We say that $R-R_{0}$ is in equivariant general position with respect to each $V_{i}, i=1, \ldots, r$, if

$$
\operatorname{dim}\left[\left(\left(R-R_{0}\right) \cap V_{i}\right)_{H}\right]_{\beta} \leqslant \operatorname{dim}\left[\left(R-R_{0}\right)_{H}\right]_{\beta}+\operatorname{dim}\left[\left(V_{i}\right)_{H}\right]_{\beta}-\operatorname{dim} M_{\beta}^{H}
$$

for every subgroup $H$ of $G$ and each component $M_{\beta}^{H}$ of $M^{H}$, and $i=1, \ldots, r$. Here $\left[P_{H}\right]_{\beta}$ denotes the part of $P_{H}$ lying in $M_{\beta}^{H}$, i.e., $\left[P_{H}\right]_{\beta}=P_{H} \cap M_{\beta}^{H}$. Observe that, since $M$ is an equivariant p.l. manifold, we have

$$
\operatorname{dim} M_{\beta}^{H}=\operatorname{dim}\left(M_{\beta}^{H}-M^{>\boldsymbol{H}}\right)=\operatorname{dim}\left[\boldsymbol{M}_{\boldsymbol{H}}\right]_{\boldsymbol{\beta}} .
$$

LeMma 2.12. Let $K$ and $L_{1}, \ldots, L_{r}$ be equivariant subpolyhedra of $D^{m}(\rho)$, and $K_{0}$ an equivariant subpolyhedron of $K$ such that $K \cap \partial D^{m}(\rho) \subset K_{0}$. Then, given $\varepsilon>0$, there exists an equivariant ambient p.l. $\varepsilon$-isotopy $\Phi$ of $D^{m}(\rho) \operatorname{rel}\left(\partial D^{m}(\rho) \cup K_{0}\right)$, such that $\Phi_{1}\left(K-K_{0}\right)$ is in equivariant general position with respect to each $L_{i}$, $i=$ $1, \ldots, r$.

Proof. Let $H_{1}, \ldots, H_{s}$ be subgroups of $G$ forming a complete set of representatives for the orbit types occurring in $K$, ordered in such a way that $\left(H_{i}\right)>\left(H_{j}\right)$ implies $i<j$. We denote

$$
K_{n}=K_{0} \cup\left(\bigcup_{i=1}^{n} G K^{H_{i}}\right), \quad n=1, \ldots, s .
$$

Each $K_{n}$ is an equivariant subpolyhedron of $D^{m}(\rho)$. We shall construct equivariant ambient p.l. $\varepsilon / s$-isotopies $\Phi^{(1)}, \ldots, \Phi^{(s)}$ and p.l. $G$-homeomorphisms $\varphi_{0}, \ldots, \varphi_{s}$ such that

1. $\Phi_{1}^{(n)} \circ \varphi_{n-1}=\varphi_{n}$,

2. $\varphi_{n}\left(K_{n}-K_{0}\right)$ is in equivariant general position with respect to each $L_{i}$, $i=1, \ldots, s$,

3. $\Phi^{(n)} \mid\left(\partial D^{m}(\rho) \cup \varphi_{n-1}\left(K_{n-1}\right)\right) \times I=\mathrm{id}$.

Define $\Phi^{(0)}=$ id and $\varphi_{-1}=\varphi_{0}$, and assume inductively that $\Phi^{(0)}, \ldots, \Phi^{(n-1)}$ and $\varphi_{0}, \ldots, \varphi_{n-1}$, where $n \geqslant 1$, have been constructed and satisfy the above conditions. Let $E$ be an equivariant triangulation of $D^{m}(\rho)$ having equivariant subcomplexes $F_{0}$ and $F$, and $L_{1}, \ldots, L_{r}$ triangulating $\varphi_{n-1}\left(K_{0}\right)$ and $\varphi_{n-1}(K)$, and $L_{1}, \ldots, L_{r}$, respectively, and such that $F_{0}$ is strongly full in $F$. Denoting $F_{j}=F_{0} \cup$ $\left(\cup_{i=1}^{j} G F^{H_{i}}\right)$ we have that $F_{j}$ triangulates $\varphi_{n-1}\left(K_{j}\right), j=1, \ldots, s$, and by Lemma $1.4 F_{j}$ is full in $F$. Now let $G v_{1}, \ldots, G v_{k}$ be the equivariant vertices of $F_{n}-F_{n-1}$ $=G\left(F^{H_{n}}-\left(F^{>H_{n}} \cup F_{0}\right)\right)$, where the ordinary vertices $v_{i}$ are chosen such that

$$
v_{1}, \ldots, v_{k} \in F^{H_{n}}-\left(F^{>H_{n}} \cup F_{0}\right) \text {. }
$$

Thus $G_{v_{i}}=H_{n}, i=1, \ldots, k$. Then we inductively construct points

$$
w_{1}, \ldots, w_{k} \in D^{m}(\rho)^{H_{n}}-\left(D^{m}(\rho)^{>H_{n}} \cup \partial D^{m}(\rho)\right),
$$


such that

(i) each $w_{i}$ does not lie in any proper hyperplane in $R^{m}(\rho)^{H_{n}}$ spanned by points from the set

$$
\operatorname{vert}\left(E^{H_{n}}\right) \cup\left[\bigcup_{\substack{j=1 \\ j \neq i}}^{k} N\left(H_{n}\right) w_{j}\right],
$$

where vert $\left(E^{H_{n}}\right)$ denotes the set of vertices of $E^{H_{n}}$.

(ii) each $w_{i}$ is so close to $v_{i}$ that, using Lemma 2.11, we have that the linear $G$-map

$$
\beta: E \rightarrow D^{m}(\rho)
$$

defined on the vertices by $\beta\left(g v_{i}\right)=g w_{i}, i=1, \ldots, k$ and $\beta(g v)=g v$ for every $v \notin G v_{i}, 1 \leqslant i \leqslant k$, and extended linearly to the simplexes of $E$, is a p.l. $G$ homeomorphism and there is an equivariant ambient p.l. $\varepsilon / s$-isotopy $\Phi^{(n)}$ $\operatorname{rel}\left(F_{n-1} \cup \partial D^{m}(\rho)\right)$ from id to $\beta$. Now define $\varphi_{n}=\Phi_{1}^{(n)} \circ \varphi_{n-1}$, thus $\beta=\varphi_{n} \circ \varphi_{n-1}^{-1}$. Now conditions 1 and 3 are satisfied and it remains to show that $\varphi_{n}\left(K_{n}-K_{0}\right)=$ $\beta\left(F_{n}-F_{0}\right)$ is in equivariant general position with respect to each $L_{i}, i=1, \ldots, r$. Since $\varphi_{n}\left|K_{n-1}=\varphi_{n-1}\right| K_{n-1}$ and since

$$
\varphi_{n}\left(K^{H_{n}}-\left(K_{0}^{H_{n}} \cup K^{>H_{n}}\right)\right)=\beta\left(F^{H_{n}}-\left(F_{0}^{H_{n}} \cup F^{>H_{n}}\right)\right)
$$

it is enough to show that

$$
\begin{aligned}
\operatorname{dim}\left[\beta \left(F^{H_{n}}-\right.\right. & \left.\left.\left(F^{>H_{n}} \cup F_{0}\right)\right) \cap L_{i}\right] \leqslant \operatorname{dim}\left(F^{H_{n}}-\left(F^{>H_{n}} \cup F_{0}\right)\right) \\
& +\operatorname{dim}\left(L_{i}^{H_{n}}-L_{i}^{>H_{n}}\right)-\operatorname{dim} D^{m}(\rho)^{H_{n}}
\end{aligned}
$$

for $i=1, \ldots, r$. Let $\sigma$ be a simplex in $F^{H_{n}}-\left(F^{>H_{n}} \cup F_{0}\right)$. Let $a_{0}, \ldots, a_{e}$, $a_{e+1}, \ldots, a_{d}$ be the vertices of $\sigma$, where $e_{e+1}, \ldots, e_{d}$ denote the ones belonging to $F^{H_{n}}-\left(F^{>H_{n}} \cup F_{0}^{H_{n}}\right)$. Since $F_{n-1}$ is full in $F_{n}$ we have that $F^{>H_{n}} \cup F_{0}^{H_{n}}$ is full in $F^{H_{n}}$ and hence we have $-1 \leqslant e \leqslant d-1$. Since $G_{a_{j}}=H_{n}, j=e+1, \ldots, d$, we have

$$
a_{j}=g_{j} v_{i j}, \quad \text { where } g_{j} \in N\left(H_{n}\right), j=e+1, \ldots, d,
$$

and moreover $v_{i_{j}} \neq v_{i^{\prime}}$ if $j \neq j^{\prime}$. We have $\beta(\sigma)=\left\langle a_{0}, \ldots, a_{e}, g_{e+1} w_{i_{e+1}}, \ldots, g_{d} w_{i_{d}}\right\rangle$.

Let $\tau=\left\langle c_{0}, \ldots, c_{t}\right\rangle$ be a simplex in $L_{i}^{H_{n}}-L_{i}^{>H_{n}}, 1<i \leqslant r$. In the case that $\beta(\sigma)$ and $\tau$ span $\mathbf{R}^{m}(\rho)^{H_{n}}$ we have

$$
\begin{aligned}
\operatorname{dim}(\beta(\sigma) \cap \tau) \leqslant & \operatorname{dim} \beta(\sigma)+\operatorname{dim} \tau-\operatorname{dim} \mathbf{R}^{m}(\rho)^{H_{n}} \\
\leqslant & \operatorname{dim}\left(F^{H_{n}}-\left(F^{>H_{n}} \cup F_{0}^{H_{n}}\right)\right) \\
& +\operatorname{dim}\left(L_{i}^{H_{n}}-L_{i}^{>H_{n}}\right)-\operatorname{dim} D^{m}(\rho)^{H_{n}}
\end{aligned}
$$

and moreover obviously

$$
\operatorname{dim}\left(\beta\left(\sigma-\sigma_{0}\right) \cap \tau\right) \leqslant \operatorname{dim}(\beta(\sigma) \cap \tau)
$$

where $\sigma_{0}=\left\langle a_{0}, \ldots, a_{e}\right\rangle$ denotes the face of $\sigma$ lying in $F>H_{n} \cup F_{0}^{H_{n}}$.

Next assume that $\beta(\sigma)$ and $\tau$ do not span $\mathbf{R}^{m}(\rho)^{H_{n}}$. It follows that the points $a_{0}, \ldots, a_{e}, g_{e+1} w_{i_{e+1}}, \ldots, g_{d-1} w_{i_{d-1}}$ and $c_{0}, \ldots, c_{t}$ span a proper hyperplane of 
$\mathbf{R}^{m}(\rho)^{H_{n}}$. Hence it follows by the construction of the points $w_{1}, \ldots, w_{k}$ that

$$
g_{d} w_{i_{d}} \notin\left\langle\left\langle a_{0}, \ldots, a_{e}, g_{e+1} w_{i_{e+1}}, \ldots, g_{d-1} w_{i_{d-1}}, c_{0}, \ldots, c_{t}\right\rangle\right\rangle \text {. }
$$

From this it follows that

$$
\left\langle a_{0}, \ldots, a_{e}, g_{e+1} w_{i_{e+1}}, \ldots, g_{d} w_{i_{d}}\right\rangle^{0} \cap\left\langle c_{0}, \ldots, c_{t}\right\rangle=\varnothing
$$

i.e.

$$
\beta(\circ) \cap \tau=\varnothing \text {. }
$$

Since $\sigma-\sigma_{0}=\cup \stackrel{\circ}{\sigma}_{i}$, where each $\sigma_{i}$ is a simplex of $F^{H_{n}}-\left(F^{>H_{n}} \cup F_{0}^{H_{n}}\right)$ such that $\beta\left(\sigma_{i}\right)$ and $\tau$ do not $\operatorname{span} \mathbf{R}^{m}(\rho)^{H_{n}}$ we now get that

$$
\beta\left(\sigma-\sigma_{0}\right) \cap \tau=\varnothing \text {. }
$$

Now (2) and (1), and (3) imply that (*) is valid. This completes the inductive step. Then observe that

$$
\Phi=\Phi^{(s)} \circ \ldots \circ \Phi^{(1)}
$$

is an equivariant ambient p.l. isotopy with the desired properties.

3. Global results. In this section we establish global versions of the results proved in $\$ 2$, prove our equivariant general position result Theorem 3.10 and deduce the $G$-imbedding results Theorems 3.11 and 3.12. In the theorems and corollaries 3.1-3.7 below $P$ denotes an equivariant polyhedron, $Q$ is an equivariant subpolyhedron of $P$ and $M$ is an equivariant p.l. manifold. In the theorems $\varepsilon$ denotes an arbitrary given invariant positive function $\varepsilon: P \rightarrow \mathbf{R}$, and in the corollaries $\varepsilon$ is a given invariant positive function $\varepsilon: P \times I \rightarrow \mathbf{R}$, which can be assumed to be such that $\varepsilon(x, t)=\varepsilon^{\prime}(x)$, for all $t \in I$, where $\varepsilon^{\prime}: P \rightarrow \mathbf{R}$.

TheOREM 3.1. Let $f: P \rightarrow M$ be a proper G-map such that $f \mid: Q \rightarrow M$ is p.l. Then there exist a proper p.l. G-map $h: P \rightarrow M$ and an equivariant proper $\varepsilon$-homotopy rel $|Q|$ from $f$ to $h$.

Corollary 3.2. Let $f_{0}, f_{1}: P \rightarrow M$ be proper p.l. G-maps and $F: P \times I \rightarrow M a$ proper G-homotopy from $f_{0}$ to $f_{1}$ such that $F \mid Q \times I$ is $p . l$. Then there exists a proper p.l. G-homotopy $F_{1}: P \times I \rightarrow M$ from $f_{0}$ to $f_{1}$, such that $F_{1}|Q \times I=F| Q \times I$ and $F_{1}$ is an $\varepsilon$-approximation to $F$.

TheOREM 3.3. Let $f: P \rightarrow M$ be an isovariant proper map such that $f \mid: Q \rightarrow M$ is p.l. Then there exist an isovariant proper p.l. map $h: P \rightarrow M$ and an equivariant proper $\varepsilon$-homotopy rel $|Q|$ from $f$ to $h$.

Corollary 3.4. Let $f_{0}, f_{1}: P \rightarrow M$ be isovariant proper p.l. maps and $F: P \times I \rightarrow$ $M$ an isovariant proper homotopy from $f_{0}$ to $f_{1}$ such that $F \mid Q \times I$ is $p . l$. Then there exists an isovariant proper p.l. homotopy $F_{1}: P \times I \rightarrow M$ from $f_{0}$ to $f_{1}$, such that $F_{1}|Q \times I=F| Q \times I$ and $F_{1}$ is an e-approximation to $F$.

TheOREM 3.5. Let $f: P \rightarrow M$ be a proper p.l. G-map such that $f \mid: Q \rightarrow M$ is isovariant (equivariantly nondegenerate). Assume that

$$
\operatorname{dim}\left(P_{\alpha}^{H}-\left(P^{>H} \cup Q\right)\right) \leqslant \operatorname{dim} M_{f(\alpha)}^{H}-\operatorname{dim} M_{f(\alpha)}^{>H}-1,
$$


for every subgroup $H$ of $G$ and each component $P_{\alpha}^{H}$ of $P^{H}$. Then there exist an isovariant (equivariantly nondegenerate) proper p.l. G-map $h: P \rightarrow M$ and an equivariant proper $\varepsilon$-homotopy rel $|Q|$ from $f$ to $h$. Moreover we can choose $h$ such that $h(P-Q) \subset$ int $M$.

Corollary 3.6. Let $f_{0}, f_{1}: P \rightarrow M$ be isovariant proper p.l. maps and $F: P \times I \rightarrow$ $M$ a proper G-homotopy from $f_{0}$ to $f_{1}$ such that $F \mid Q \times I$ is isovariant and p.l. Assume that

$$
\operatorname{dim}\left(P_{\alpha}^{H}-\left(P^{>H} \cup Q\right)\right) \leqslant \operatorname{dim} M_{f(\alpha)}^{H}-\operatorname{dim} M_{f(\alpha)}^{>H}-2
$$

for every subgroup $H$ of $G$ and each component $P_{\alpha}^{H}$ of $P^{H}$. Then there exists an isovariant proper $p . l$. homotopy $F_{1}: P \times I \rightarrow M$ from $f_{0}$ to $f_{1}$, such that $F_{1} \mid Q \times I=$ $F \mid Q \times I$ and $F_{1}$ is an e-approximation to $F$.

THEOREM 3.7. Let $f: P \rightarrow M$ be an isovariant proper p.l. map such that $f \mid: Q \rightarrow M$ is equivariantly nondegenerate. Assume that

$$
\operatorname{dim}\left(P_{\alpha}^{H}-\left(P^{>H} \cup Q\right)\right) \leqslant \operatorname{dim} M_{f(\alpha)}^{H},
$$

for every subgroup $H$ of $G$ and each component $P_{\alpha}^{H}$ of $P^{H}$. Then there exist an equivariantly nondegenerate proper p.l. G-map $h: P \rightarrow M$ and an isovariant proper $\varepsilon$-homotopy rel| $Q \mid$ from $f$ to $h$. Moreover we can choose $h$ such that $h(P-Q) \subset$ int $M$.

REMARK. Observe that Theorems 3.1 and 3.5 in particular give us the following result: Let $f: P \rightarrow M$ be a proper $G$-map and assume that

$$
\operatorname{dim}\left(P_{\alpha}^{H}-P{ }^{>H}\right) \leqslant \operatorname{dim} M_{f(\alpha)}^{H}-\operatorname{dim} M_{f(\alpha)}^{>H}-1
$$

for every subgroup $H$ of $G$ and each component $P_{\alpha}^{H}$ of $P^{H}$. Then $f$ is equivariantly $\varepsilon$-homotopic, by a proper homotopy, to an isovariant proper (p.l.) map $h: P \rightarrow M$.

The proofs of the above four theorems are completely similar to each other, using Propositions 2.2, 2.4, 2.6 and 2.9, respectively. In all four cases the proof consists of a patching together process of the corresponding local result, in order to get the global result. (Compare also with corresponding proofs in the ordinary nonequivariant case, e.g. the proof of Lemma 4.2 in Hudson [6] or the proof of Theorem 1.6.10, Part I in Rushing [12].) We give the proof for the case of Theorem 3.5, being the one that looks most complicated. The Corollaries 3.2 and 3.4 are easy consequences of the corresponding theorems.

Proof of Corollary 3.6. Since $f_{0}, f_{1}$ and $F \mid Q \times I$ are p.l. we have by Corollary 3.2 that there exists a proper p.l. $G$-homotopy $F^{\prime}: P \times I \rightarrow M$ from $f_{0}$ to $f_{1}$ such that $F^{\prime}|Q \times I=F| Q \times I$ and $F^{\prime}$ is an $\varepsilon / 2$-approximation to $F$. Now the proper p.l. $G$-map $F^{\prime}: P \times I \rightarrow M$ is such that $F^{\prime} \mid(P \times\{0,1\} \cup Q \times I)$ is isovariant and hence, using the dimension assumption, it follows by Theorem 3.5 that there exists an isovariant proper p.l. map $F_{1}: P \times I \rightarrow M$ such that $F_{1} \mid(P \times\{0,1\} \cup Q \times I)$ $=F^{\prime} \mid(P \times\{0,1\} \cup Q \times I)$ and $F_{1}$ is an $\varepsilon / 2$-approximation to $F^{\prime}$. Then $F_{1}$ is as desired.

Furthermore we have the following strengthened versions of Theorems 3.5 and 3.7. 
THEOREM 3.5' (3.7'). Everything as in Theorem 3.5 (3.7) but with the added conclusion that the homotopy from $f$ to $h$ moreover is p.l.

Proof. This follows from Theorem 3.5 and Corollary 3.2 (Theorem 3.7 and Corollary 3.4).

Proof of Theorem 3.5. We can assume that $\varepsilon: M \rightarrow R$ is such that if $F$ : $P \times I \rightarrow M$ is any $\varepsilon$-homotopy from $f$ to some map $h$ then $F$ and hence also $h$ are proper maps. Now choose equivariant triangulations of $Q \subset P$ and $M$ such that $f$ is simplicial. Denote these triangulations again by $Q, P$ and $M$. Let $G v_{1}, G v_{2}, \ldots$ be the equivariant vertices of $M$. Let us denote $B_{j}=(m+1) /(m+2) \cdot \operatorname{St}\left(v_{j}, M\right)$, where $m=\operatorname{dim} M, j=1, \ldots($ Here $(m+1) /(m+2) \cdot \operatorname{St}(v, M)$ denotes the set of points $x \in s<\operatorname{St}(v, M)$ such that the $v$-coordinate $x_{v}$ of $x$ in $s$ satisfies $1 /(m+2) \leqslant x_{v} \leqslant 1$.) We have $M=\cup_{j=1}^{\infty}$ Int $G B_{j}$. Observe that if $x \in B_{j}$ then $G_{x} \subset G_{v_{j}}$. Let $P^{0}$ and $M^{0}$ be equivariant subdivisions of $P$ and $M$, respectively, such that for any equivariant simplex $A$ of $P^{0}$ we have $f(A) \subset \operatorname{Int} G B_{j}$, for some $j$, and each $G B_{j}, j=1, \ldots$, is an equivariant subcomplex of $M^{0}$ and $f: P^{0} \rightarrow M^{0}$ is simplicial. Let $A_{1}, A_{2}, \ldots$ be the equivariant simplexes of $P^{0}-Q^{0}$ ordered in such a way that an equivariant simplex follows all its faces $\left(Q^{0}\right.$ denotes the equivariant subdivision of $Q$ induced by the subdivision $P^{0}$ of $P$ ). We denote

$$
P_{n}^{0}=Q^{0} \cup\left(\bigcup_{j=1}^{n} A_{j}\right), \quad n=1, \ldots
$$

Each $P_{n}^{0}$ is an equivariant subcomplex of $P^{0}$ and $\cup_{n=1}^{\infty} P_{n}^{0}=P$, and $P_{0}^{0}=Q^{0}$ by definition. We shall inductively define equivariant subdivisions $P^{i}$ of $P^{0}$ and $M^{i}$ of $M^{0}$ and simplicial $G$-maps $f_{i}: P^{i} \rightarrow M^{i}, i=1, \ldots$, such that the following conditions are satisfied. (By $P_{n}^{i}$ we denote the equivariant subdivision of $P_{n}^{0}$ induced from the subdivision $P^{i}$ of $P^{0}$.)

1. $f_{i} \mid: P_{i}^{i} \rightarrow M^{i}$ is isovariant (equivariantly nondegenerate).

2. $f_{i}$ is equivariantly $\left(\varepsilon / 2^{i}\right)$-homotopic rel $\left|P_{i-1}\right|$, by a proper homotopy $F^{(i)}$, to $f_{i-1}, i \geqslant 1$.

3. $f_{i}\left(\left|P_{i}\right|-|Q|\right) \subset$ int $M$.

4. If $A$ is an equivariant simplex of $P^{0}$ then $f_{i}(A) \subset$ Int $G B_{j}$, for some $j$.

Let $f_{0}=f: P^{0} \rightarrow M^{0}$ and assume inductively that $f_{0}, \ldots, f_{i-1}$ have been defined and satisfy the above conditions. We have $f_{i-1}\left(A_{i}\right) \subset$ Int $G B_{j(i)}$, for some $j(i)$. Let $A_{i}=G s_{i}$, where $s_{i}$ is an ordinary simplex in $P^{0}$, and we have chosen $s_{i}$ such that $f_{i-1}\left(s_{i}\right) \subset \operatorname{Int} B_{j(i)}=\operatorname{Int}\left((m+1) /(m+2) \cdot \operatorname{St}\left(v_{j(i)}, M\right)\right)$. Let us for simplicity denote $A_{i}=A, s_{i}=s, B_{j(i)}=B$ and $v_{j(i)}=v$. Let $K_{0}$ be the maximal isotropy subgroup of $s$ and let us denote $G_{v}=H$. We have $K_{0} \subset H$.

Let us denote $S=S_{i}=f_{i-1}\left(N\left(|G s|, P^{i-1}\right)\right)$ and $R=R_{i}=f_{i-1}^{-1}\left(S_{i}\right)$ and let $R^{\prime}$ and $S^{\prime}$ be equivariant subdivisions of $R$ and $S$, respectively, such that $f_{i-1}\left(N\left(|G s|, R^{\prime}\right)\right) \subset$ Int $G B$ and $f_{i-1}: R^{\prime} \rightarrow S^{\prime}$ is simplicial. Let $\tilde{M}^{i-1}$ be the standard extension (see [8, p. 202]) of the equivariant subdivision $S^{\prime}$ of $S$ to an equivariant subdivision of $M^{i-1}$. Let $\tilde{P}^{i-1}$ also be a standard extension of the equivariant subdivision $R^{\prime}$ of $R$ to an equivariant subdivision of $P^{i-1}$, but this 
time, instead of starring at the barycenters, starring the simplexes $\sigma$ of $\stackrel{\circ}{N}\left(R, P^{i-1}\right)$ $-R$ at an interior point of $\left(f_{i-1} \mid \sigma\right)^{-1} b\left(f_{i-1}(\sigma)\right)$ and $G$-translates $g \sigma$ at corresponding points. Then $f_{i-1}: \tilde{P}^{i-1} \rightarrow \tilde{M}^{i-1}$ is simplicial, $\tilde{P}^{i-1}$ equals $P^{i-1}$ outside $\stackrel{\circ}{N}\left(R, P^{i-1}\right)$ and $\tilde{M}^{i-1}$ equals $M^{i-1}$ outside $\stackrel{\circ}{N}\left(S, M^{i-1}\right)$, and

$$
f_{i-1}\left(N\left(|G s|, \tilde{P}^{i-1}\right)\right) \subset \text { Int } G B .
$$

We then have $f_{i-1}\left(N\left(|H s|, \tilde{P}^{i-1}\right)\right) \subset$ Int $B$ and hence $g N\left(|H s|, \tilde{P}^{i-1}\right) \cap$ $N\left(|H s|, \tilde{P}^{i-1}\right)=\varnothing$ if $g \notin H$. It follows that

$$
N\left(|G s|, \tilde{P}^{i-1}\right)=G N\left(|H s|, \tilde{P}^{i-1}\right) \cong G \times_{H} N\left(|H s|, \tilde{P}^{i-1}\right) .
$$

Let $\beta: B \rightarrow D^{m}(\tau)$, where $\tau: H \rightarrow O(m)$ is an orthogonal representation of $H$, be a p.l. $H$-homeomorphism. Now consider the $H$-map $\beta \circ\left(f_{i-1} \mid\right): N\left(|H s|, \tilde{P}^{i-1}\right) \rightarrow$ $D^{m}(\tau)$. Take $H$-equivariant subdivisions $N\left(|H s|, \tilde{P}^{i-1}\right)^{*}$ and $B^{*}$ (where $B$ is considered as a subcomplex of $\left.\tilde{M}^{i-1}\right)$, and $D^{m}(\tau)^{*}$ such that

$$
\beta \circ\left(f_{i-1} \mid\right): N\left(|H s|, \tilde{P}^{i-1}\right)^{*} \rightarrow D^{m}(\tau)^{*}
$$

is a simplicial $H$-map and $\beta: B^{*} \rightarrow D^{m}(\tau)^{*}$ is a simplicial $H$-homeomorphism. Let us denote

$$
X=N\left(|H s|, \tilde{P}^{i-1}\right)^{*}
$$

and let $Y_{1}, Y_{2}$ and $Y_{3}$ be the $H$-equivariant subcomplexes of $X$ given by $\left|Y_{1}\right|=|H s|$ and $\left|Y_{2}\right|=|X| \cap\left|P_{i-1}\right|$ and $\left|Y_{3}\right|=\operatorname{Bd}_{|P|}|X|$ Let $K$ be an arbitrary subgroup of $H$ and let $\left(Y_{1}^{K}\right)_{\alpha}$ be some component of $Y_{1}^{K}$, and denote by $P_{\alpha}^{K}$ the corresponding component of $P^{K}$. Then we have $\left(Y_{1}^{K}\right)_{\alpha}-\left(Y_{1}^{>K} \cup Y_{2}\right) \subset P_{\alpha}^{K}-\left(P^{>K} \cup Q\right)$ and hence

$$
\operatorname{dim}\left(\left(Y_{1}^{K}\right)_{\alpha}-\left(Y_{1}^{>K} \cup Y_{2}\right)\right) \leqslant \operatorname{dim}\left(P_{\alpha}^{K}-(P>K \cup Q)\right) .
$$

Let $x \in\left(Y_{1}^{K}\right)_{\alpha}$, then $f_{i-1}(x) \in B^{K}$ and $B^{K}$ is connected. Since $f_{i-1}$ is $G$-homotopic to $f$ it follows that $f_{i-1}(x)$ and $f(x)$ lie in the same component of $M^{K}$, i.e., $f_{i-1}(x) \in M_{f(\alpha)}^{K}$. Hence we have $B^{K} \subset M_{f(\alpha)}^{K}$, and therefore

$$
\operatorname{dim} M_{f(\alpha)}^{K}=\operatorname{dim} B^{K} .
$$

Since $B^{>K} \subset M_{f(\alpha)}^{>K}$ we have $\operatorname{dim} B^{>K} \leqslant M_{f(\alpha)}^{>K}$ and hence

$$
\operatorname{dim} M_{f(\alpha)}^{K}-\operatorname{dim} M_{f(\alpha)}^{>K} \leqslant \operatorname{dim} B^{K}-\operatorname{dim} B^{>K} .
$$

By the dimension assumption in Theorem 3.5 and (3) and (4) we now get

$$
\operatorname{dim}\left(\left(Y_{1}^{K}\right)_{\alpha}-\left(Y_{1}^{>K} \cup Y_{2}\right)\right) \leqslant \operatorname{dim} D^{m}(\tau)^{K}-\operatorname{dim} D^{m}(\tau)^{>K}-1 .
$$

Since this holds for every component $\left(Y_{1}^{K}\right)_{\alpha}$ of $Y_{1}^{K}$ it follows that in fact

$$
\operatorname{dim}\left(Y_{1}^{K}-\left(Y_{1}^{>K} \cup Y_{2}\right)\right) \leqslant \operatorname{dim} D^{m}(\tau)^{K}-\operatorname{dim} D^{m}(\tau)^{>K}-1
$$

for every subgroup $K$ of $H$.

Now since $\left|Y_{2}\right| \subset\left|P_{i-1}\right|$ we have that the simplicial $H$-map

$$
\beta \circ\left(f_{i-1} \mid\right): X \rightarrow D^{m}(\tau)^{*}
$$

is such that $\beta \circ\left(f_{i-1} \mid\right) \mid Y_{2}$ is isovariant (equivariantly nondegenerate). Thus by Proposition 2.6 there exist equivariant subdivisions $X^{*}$ and $D^{m}(\rho)^{* *}$ of $X$ and 
$D^{m}(\rho)^{*}$, respectively, and a simplicial $H$-map

$$
h_{1}: X^{*} \rightarrow D^{m}(\tau)^{* *}
$$

such that $h_{1} \mid\left(Y_{1} \cup Y_{2}\right)^{*}$ is isovariant (equivariantly nondegenerate) and $h_{1}$ is $H$-equivariantly $\varepsilon_{1}$-homotopic rel $\left|Y_{2} \cup Y_{3}\right|$ to $\beta \circ\left(f_{i-1} \mid\right)$, for any given $\varepsilon_{1}>0$, and moreover $h_{1}\left(Y_{1}-Y_{2}\right) \subset \dot{D}^{m}(\tau)$. Now consider the simplicial $H$-map

$$
h=\beta^{-1} \circ h_{1}: X^{*} \rightarrow B^{* *}
$$

and its $G$-extension

$$
\bar{h}=\mathrm{id} \times_{H} h: G \times_{H} X^{*} \rightarrow G \times_{H} B^{* *} \cong G B^{* *} .
$$

By (1) we have

$$
\bar{h}: N\left(|G s|, \tilde{P}^{i-1}\right)^{* *} \rightarrow G B^{* *} .
$$

Observe that $\vec{h}(x)=f_{i-1}(x)$ for every $x \in \mathrm{Bd}_{|P|}\left|N\left(|G s|, \tilde{P}^{i-1}\right)\right|$. We define

$$
f_{i}:|P| \rightarrow|M|
$$

by

$$
\begin{gathered}
f_{i}|| N\left(|G s|, \tilde{P}^{i-1}\right) \mid=\bar{h}, \\
f_{i}|| P\left|-\operatorname{Int}_{|P|}\right| N\left(|G s|, \tilde{P}^{i-1}\right)\left|=f_{i-1}\right||P|-\operatorname{Int}_{|P|}\left|N\left(|G s|, \tilde{P}^{i-1}\right)\right| .
\end{gathered}
$$

This gives us a well-defined $G$-map $f_{i}:|P| \rightarrow|M|$. Let $L=L_{j(i)}=f_{i-1}^{-1}\left(G B_{j(i)}\right)$, then $L$ is an equivariant subcomplex of $\tilde{P}^{i-1}$. Now extend the equivariant subdivision $N\left(|G s|, \tilde{P}^{i-1}\right)^{* *}$ of $N\left(|G s|, \tilde{P}^{i-1}\right)$ to an equivariant subdivision $L^{* *}$ of $L$ such that $f_{i} \mid: L^{* *} \rightarrow G B^{* *}$ is simplicial. Let $M^{i}$ be the standard extension of the equivariant subdivision $G B^{* *}$ of $G B$ to an equivariant subdivision of $\tilde{M}^{i-1}$. Let $P^{i}$ be a standard extension of the equivariant subdivision $L^{* *}$ to an equivariant subdivision of $\tilde{P}^{i-1}$ chosen such that $f_{i}: P^{i} \rightarrow M^{i}$ is simplicial. Then $P^{i-1}$ equals $\tilde{P}^{i-1}$ outside $\stackrel{\circ}{N}\left(L, \tilde{P}^{i-1}\right)$ and $M^{i}$ equals $\tilde{M}^{i-1}$ outside $\stackrel{\circ}{N}\left(G B, \tilde{M}^{i-1}\right)$ and $f_{i}$ equals $f_{i-1}$ outside $\stackrel{\circ}{N}\left(L, \tilde{P}^{i-1}\right)$. Choosing $\varepsilon_{1}>0$ small enough we see that $f_{i}: P^{i} \rightarrow M^{i}$ satisfies conditions 1-4.

To conclude the proof we show that we can take $M^{\prime}=\lim _{i \rightarrow \infty} M^{i}, P^{\prime}=$ $\lim _{i \rightarrow \infty} P^{i}$ and $h=\lim _{i \rightarrow \infty} f_{i}$ and that then $h: P^{\prime} \rightarrow M^{\prime}$ is a proper, isovariant (equivariantly nondegenerate) simplicial map such that $h(|P|-|Q|) \subset$ int $M$ and $h$ is equivariantly $\varepsilon$-homotopic rel $|Q|$ to $f$. We have that $P^{i}$ equals $P^{i-1}$ and $f_{i}$ equals $f_{i-1}$ outside $\stackrel{\circ}{N}\left(R_{i}, P^{i-1}\right) \cup \stackrel{\circ}{N}\left(L_{j(i)}, \tilde{P}^{i-1}\right)$ and $M^{i}$ equals $M^{i-1}$ outside $\stackrel{\circ}{N}\left(S_{i}, M^{i-1}\right)$ $\cup N\left(G B_{j(i)}, \tilde{M}^{i-1}\right)$. Observe that for fixed $j_{0}$ we have $j(i)=j_{0}$ for only finitely many $i$. For if $f_{i-1}\left(A_{i}\right) \subset G B_{j_{0}}$ then $f\left(A_{i}\right) \subset G \cdot \operatorname{St}\left(v_{j_{0}}, M\right)$, assuming $\varepsilon(x)<$ $1 /(m+2)$ for every $x \in|P|$, and, since $f$ is proper, $f^{-1}\left(G \cdot \operatorname{St}\left(v_{j_{0}}, M\right)\right)$ is compact and hence meets only finitely many $A_{i}$. It is now immediately seen that any compact set $C$ in $P$ or in $M$ intersects the sets $N\left(R_{i}, P^{i-1}\right) \cup N\left(L_{j(i)}, \tilde{P}^{i-1}\right)$ or the sets $N\left(S_{i}, M^{i-1}\right) \cup N\left(G B_{j(i)}, \tilde{M}^{i-1}\right)$, respectively, for only finitely many $i$. It follows that $P^{\prime}=\lim _{i \rightarrow \infty} P^{i}$ and $M^{\prime}=\lim _{i \rightarrow \infty} M_{i}^{i}$ exist and it is then immediately seen that $h=\lim _{i \rightarrow \infty} f_{i}: P^{\prime} \rightarrow M^{\prime}$ has the desired properties.

Since each $F^{(i)}, i \geqslant 1$, is an equivariant $\varepsilon / 2^{i}$-homotopy rel $P_{i-1}$ from $f_{i-1}$ to $f_{i}$ it follows (by a standard argument) that the map $F: P \times I \rightarrow M$ defined by 


$$
\begin{gathered}
F(x, t)=F^{(i)}\left(x, \frac{t-(1-1 / i)}{1 / i-1 /(i+1)}\right) \text { for } 1-1 / i \leqslant t \leqslant 1-1 /(i+1), i>1, \\
F(x, 1)=h(x),
\end{gathered}
$$

is a well-defined continuous $G$-homotopy from $f$ of $h$. Moreover $F$ is $\operatorname{rel}|Q|$ and since $F$ is an $\varepsilon$-homotopy it follows that $F$ is proper.

Our next result shows that any equivariant subpolyhedron $M$ can be moved, by an equivariant ambient isotopy, into equivariant general position with respect to any finite number of equivariant subpolyhedra of $M$. This result is the global version of Lemma 2.12 .

Proposition 3.8. Let $R$ and $V_{1}, \ldots, V_{r}$ be equivariant subpolyhedra of the equivariant p.l. manifold $M$, and $R_{0}$ an equivariant subpolyhedron of $R$ such that $R \cap \partial M \subset R_{0}$. Let $\varepsilon: M \rightarrow \mathbf{R}_{+}$be an invariant positive function. Then there exists an equivariant ambient p.l. $\varepsilon$-isotopy $\Psi$ of $M \operatorname{rel}\left(\partial M \cup R_{0}\right)$ such that $\Psi_{1}\left(R-R_{0}\right)$ is in equivariant general position with respect to each $V_{k}, k=1, \ldots, r$.

Proof. Triangulate $M$ and use $M$ again to denote this equivariant combinatorial manifold. Let $G v_{1}, G v_{2}, \ldots$ be the equivariant vertices of $M$ and denote $B_{j}=$ $(m+1) /(m+2) \cdot \operatorname{St}\left(v_{j}, M\right)$. Then each $B_{j}$ is a $G_{v_{j}}$-equivariant subpolyhedron of $M$ and

$$
G B_{j} \cong G \times_{G_{v_{j}}} B_{j}, \quad j=1, \ldots
$$

Each $G B_{j}$ is a $G$-equivariant subpolyhedron of $M$ and $M=\cup_{j=1}^{\infty}$ Int $G B_{j}$. Consider $R$ triangulated such that $R_{0}$ is an equivariant subcomplex of $R$ and such that for any equivariant simplex $A$ of $R$ we have $A \subset \operatorname{Int} G B_{j}$, for some $j$. Let $A_{1}, A_{2}, \ldots$ be the equivarient simplexes of $R-R_{0}$ ordered in such a way that an equivariant simplex follows all its faces. Let us denote $R_{i}=R_{0} \cup \cup_{j=1}^{i} A_{j}$. We shall define p.l. homeomorphisms $h_{i}, i=0,1, \ldots$, of $M$ and equivariant ambient p.l. isotopies $\Psi^{(i)}, i=1,2, \ldots$, of $M \operatorname{rel}\left(\partial M \cup R_{0}\right)$ such that

1. $\Psi_{1}^{(i)} \circ h_{i-1}=h_{i}$.

2. $\Psi^{(i)}$ is an $\left(\varepsilon / 2^{i}\right)$-isotopy.

3. If $A$ is an equivariant simplex of $R$ then $h_{i}(A) \subset$ Int $G B_{j}$, for some $j$.

4. $h_{i}\left(R_{i}-R_{0}\right)$ is in equivariant general position with respect to each $V_{k}, k=$ $1, \ldots, r$.

In order to start an induction define $h_{-1}=h_{0}=\mathrm{id}$ and $\Psi^{(0)}=\mathrm{id}$, and then assume that $h_{0}, \ldots, h_{i-1}$ and $\Psi^{(0)}, \ldots, \Psi^{(i-1)}$, where $i>1$, have been defined and satisfy the above conditions. Thus we have $h_{i-1}\left(A_{i}\right) \subset \operatorname{Int} G B_{j(i)}$, for some $j(i)$. Let us for simplicity denote $A_{i}=A$ and $B_{j(i)}=B$. Let $A=G s$, where $s$ is an ordinary simplex in $R$ chosen such that $h_{i-1}(s) \subset$ Int $B$.

Denote $G_{v}=H$, where $v=v_{j(i)}$, and let $\beta: B \rightarrow D^{m}(\rho)$ be a p.l. $H$-homeomorphism, where $\rho: H \rightarrow O(m)$ is an orthogonal representation of $H$. Let $K_{0}=$ $\beta\left(h_{i-1}\left(R_{i-1}\right) \cap B\right)$ and $K=\beta\left(h_{i-1}\left(R_{i}\right) \cap B\right)$ and $L_{k}=\beta\left(V_{k} \cap B\right), k=1, \ldots, r$. Then $K_{0}, K$ and $L_{1}, \ldots, L_{r}$ are $H$-equivariant subpolyhedra of $D^{m}(\rho)$, and $K \cap$ $\partial D^{m}(\rho) \subset K_{0}$. By Lemma 2.12 there exists, for any given $\varepsilon_{1}>0$, an equivariant ambient p.l. $\varepsilon_{1}$-isotopy $\Phi$ of $D^{m}(\rho) \operatorname{rel}\left(\partial D^{m}(\rho) \cup K_{0}\right)$ such that $\Phi_{1}\left(K-K_{0}\right)$ is in 
equivariant general position with respect to each $L_{1}, \ldots, L_{r}$. Using the fact that $G B \times I \cong G \times_{H}(B \times I)$ we now define $\Psi^{(i)}$ by

$$
\begin{gathered}
\Psi^{(i)} \mid G B \times I=\mathrm{id} \times{ }_{H}\left[\left(\beta^{-1} \times \mathrm{id}\right) \circ \Phi \circ(\beta \times \mathrm{id})\right], \\
\Psi^{(i)} \mid(M-\text { Int } G B) \times I=\mathrm{id} .
\end{gathered}
$$

Then define $h_{i}=\Psi_{1}^{(i)} \circ h_{i-1}$. Observe that since the $G$-homeomorphism $h_{i}$ is $G$-homotopic to id we have

$$
\left[\left(h_{i}\left(R_{i}-R_{0}\right)\right)_{F}\right]_{\gamma}=h_{i}\left(\left[\left(T_{i}-R_{0}\right)_{F}\right]_{\gamma}\right)
$$

for any subgroup $F$ of $G$ and any component $M_{\gamma}^{F}$ of $M^{F}$. It follows that in order to establish condition 4 for $h_{i}$ we must show that

$$
\operatorname{dim}\left(h_{i}\left(\left[\left(R_{i}-R_{0}\right)_{F}\right]_{\gamma}\right) \cap V_{k}\right) \leqslant \operatorname{dim}\left[\left(R_{i}-R_{0}\right)_{F}\right]_{\gamma}+\operatorname{dim}\left[\left(V_{k}\right)_{F}\right]_{\gamma}-\operatorname{dim} M_{\gamma}^{F},
$$

for every subgroup $F$ of $G$ and each component $M_{\gamma}^{F}$ of $M^{F}$, and $k=1, \ldots, r$. The fact that $h_{i-1}\left(R_{i-1}-R_{0}\right)$ is in equivariant general position with respect to each $V_{k}$, $k=1, \ldots, r$, gives us

$$
\begin{aligned}
\operatorname{dim}\left(h _ { i - 1 } \left(\left[\left(R_{i-1}-\right.\right.\right.\right. & \left.\left.\left.\left.R_{0}\right)_{F}\right]_{\gamma}\right) \cap V_{k}\right) \\
& \leqslant \operatorname{dim}\left[\left(R_{i-1}-R_{0}\right)_{F}\right]_{\gamma}+\operatorname{dim}\left[\left(V_{k}\right)_{F}\right]_{\gamma}-\operatorname{dim} M_{\gamma}^{F},
\end{aligned}
$$

for every subgroup $F$ of $G$ and each component $M_{\gamma}^{F}$ of $M^{F}$, and $k=1, \ldots, r$. Since $R_{i}-R_{0}=\left(R_{i}-R_{i-1}\right) \cup\left(R_{i-1}-R_{0}\right)$ and $h_{i}\left|R_{i-1}=h_{i-1}\right| R_{i-1}$, and $R_{i}-$ $R_{i-1}=\AA_{i}=G s$, it now follows that it is enough to prove that

$$
\operatorname{dim}\left(h_{i}\left(\left[\stackrel{\circ}{s}_{F}\right]_{\gamma}\right) \cap V_{k}\right) \leqslant \operatorname{dim}\left[\stackrel{\circ}{s}_{F}\right]_{\gamma}+\operatorname{dim}\left[\left(V_{k}\right)_{F}\right]_{\gamma}-\operatorname{dim} M_{\gamma}^{F}
$$

for every subgroup $F$ of $G$ and any component $M_{\gamma}^{F}$ of $M^{F}$, and $k=1, \ldots, r$. Let $H^{\prime}$ denote the principal isotropy subgroup of $s$, then $H^{\prime} \subset H$. Now if $F \neq H^{\prime}$ we have $i_{F}=\varnothing$ and there is nothing to prove. Thus we need only consider the case $F=H^{\prime}$. Then $\dot{s}_{H^{\prime}}=\dot{s}$. If $\left[s_{\gamma}\right]_{\gamma}=\varnothing$ there is again nothing to prove. Thus we can

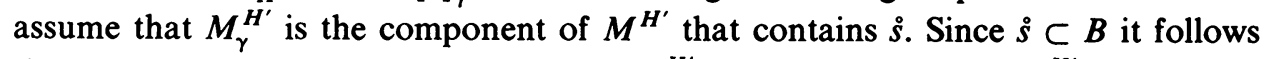
that $s$ belongs to the same component of $M^{H^{\prime}}$ as $v=v_{j(i)}$, i.e., $v \in M_{\gamma}^{H^{\prime}}$. Hence

$$
\operatorname{dim} M_{\gamma}^{H^{\prime}}=\operatorname{dim} B^{H^{\prime}}=\operatorname{dim} D^{m}(\rho)^{H^{\prime}} .
$$

We have $\beta h_{i-1}(H s)=K-K_{0} \subset D^{m}(\rho)$ and $h_{i}(H s)=\beta^{-1} \Phi_{1} \beta h_{i-1}(H s)$. Thus we get

$$
\begin{aligned}
\operatorname{dim}\left(h_{i}\left(\stackrel{\circ}{s} \cap \cap V_{k}\right)\right. & =\operatorname{dim}\left(h_{i}(H s)_{H^{\prime}} \cap V_{k}\right)=\operatorname{dim}\left(\Phi_{1}\left(K-K_{0}\right)_{H^{\prime}} \cap L_{k}\right) \\
& \leqslant \operatorname{dim}\left(K-K_{0}\right)_{H^{\prime}}+\operatorname{dim}\left(L_{k}\right)_{H^{\prime}}-\operatorname{dim} D^{m}(\rho)^{H^{\prime}} \\
& \leqslant \operatorname{dim} s+\operatorname{dim}\left[\left(V_{k}\right)_{H^{\prime}}\right]-\operatorname{dim} M_{\gamma}^{H^{\prime}},
\end{aligned}
$$

for $k=1, \ldots, r$. This completes the proof of the fact that $h_{i}$ satisfies condition 4 .

Choosing $\varepsilon_{1}>0$ small enough conditions 2 and 3 will be satisfied. Finally observe that any compact subset of $M \times I$ intersects only finitely many sets $G B_{j} \times I$. Also each $\Psi^{(i)}$ equals the identity outside Int $G B_{j(i)} \times I$ and for fixed $j_{0}$ there are only finitely many $i$ such that $j(i)=j_{0}$. Thus given a finite collection $G B_{j_{1}} \times I, \ldots, G B_{j_{s}} \times I$ there exists an $i_{0}$ such that for any $i>i_{0}$ we have that $\Psi^{(i)}$ 
equals the identity outside $\cup_{k=1}^{s}$ Int $G B_{j_{k}} \times I$. It follows that

$$
\Psi=\lim _{i \rightarrow \infty} \Psi^{(i)} \circ \cdots \circ \Psi^{(1)}
$$

is a well-defined equivariant ambient p.l. isotopy of $M$ which satisfies the conclusions of the proposition.

Definition. Let $K$ be an equivariant simplicial complex and $L$ an equivariant subcomplex of $K$ and $f: K \rightarrow M$ an equivariantly nondegenerate proper p.l. $G$-map, where $M$ is an equivariant p.l. manifold. Let $V_{1}, \ldots, V_{r}$ be equivariant subpolyhedra of $M$. We say that $f \mid: K-L \rightarrow M$ is in equivariant strong general position with respect to each $V_{j}, j=1, \ldots, r$, if the following condition is satisfied. For any subgroup $H$ of $G$ and for any component $M_{\beta}^{H}$ of $M^{H}$ the following holds: Let $s_{1}, \ldots, s_{n}$ (any $n \geqslant 1$ ), be any distinct simplexes of $K-L$ with principal isotropy subgroup equal to $H$ such that $f\left(s_{i}\right) \subset M_{\beta}^{H}$ for $i=1, \ldots, n$. Then

$$
\operatorname{dim}\left[\left(\bigcap_{i=1}^{n} f\left(s_{i}\right)\right) \cap V_{j}\right] \leqslant \sum_{i=1}^{n} \operatorname{dim} s_{i}+\operatorname{dim}\left[\left(V_{j}\right)_{H}\right]_{\beta}-n \cdot \operatorname{dim} M_{\beta}^{H}
$$

for $j=1, \ldots, r$. Here $\left[V_{H}\right]_{\beta}=V_{H} \cap M_{\beta}^{H}$.

Observe that if $s$ and $s^{\prime}$ are simplexes with principal isotropy subgroups $H$ and $H^{\prime}$, respectively, where $H \neq H^{\prime}$, then $f\left(s^{\circ}\right) \cap f\left(s^{\prime}\right)=\varnothing$, since $f$ in particular is isovariant. Also, in case $s$ and $s^{\prime}$ have principal isotropy subgroup $H$ but $f\left(s^{\circ}\right) \subset M_{\beta}^{H}$

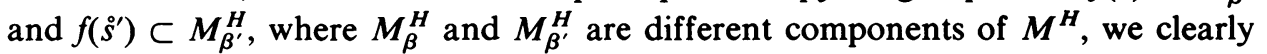
have $f\left(s^{\circ}\right) \cap f\left(s^{\prime}\right)=\varnothing$. Hence the situation considered in the definition is the only relevant one.

If $f \mid: K-L \rightarrow M$ is in equivariant strong general position with respect to $V=M$ we simply say that $f \mid: K-L \rightarrow M$ is in equivariant strong general position, and this means that, under the same conditions as above, we have

$$
\operatorname{dim}\left[\bigcap_{i=1}^{n} f\left(\dot{s}_{i}\right)\right] \leqslant \sum_{i=1}^{n} \operatorname{dim} s_{i}-(n-1) \operatorname{dim} M_{\beta}^{H}
$$

Proposition 3.9. Let $P^{*}$ be an equivariant simplicial complex and $Q^{*}$ an equivariant subcomplex of $P^{*}$, and let $V_{1}, \ldots, V_{r} \subset M$ and $\varepsilon: P \rightarrow \mathbf{R}_{+}$be as before. Assume that $f: P^{*} \rightarrow M$ is an equivariantly nondegenerate proper p.l. G-map such that $f(P-Q) \subset$ int $M$. Then there exists an isovariant proper p.l. $\varepsilon$-homotopy rel $Q$ from $f$ to an equivariantly nondegenerate proper p.l. G-map $h: P^{*} \rightarrow M$, where $h(P-Q) \subset$ int $M$, and such that $h \mid: P^{*}-Q^{*} \rightarrow M$ is in equivariant strong general position with respect to $V_{j}, j=1, \ldots, r$.

Proof. We can assume that $\varepsilon$ is such that any $\varepsilon$-homotopy $F$ from $f$ to some other map $h$ is proper. Let $A_{1}, A_{2}, \ldots$ be the equivariant simplexes of $P^{*}-Q^{*}$ in such an order that an equivariant simplex follows all its faces. Denote $P_{n}^{*}=Q^{*} \cup$ $\cup_{j=1}^{n} A_{j}$. We shall inductively define equivariantly nondegenerate proper p.l. $G$-maps $f_{n}: P^{*} \rightarrow M, n \geqslant 0$, and isovariant homotopies $F^{(n)}: P \times I \rightarrow M, n>1$, such that

(1) $F^{(n)}$ is an $\varepsilon / 2^{n+1}$-homotopy rel $P_{n-1}$ from $f_{n-1}$ to $f_{n}$. 
(2) $f_{n}(P-Q) \subset$ int $M$.

(3) $f_{n} \mid: P_{n}^{*}-Q^{*} \rightarrow M$ is in equivariant strong general position with respect to each $V_{j}, j=1, \ldots, r$.

Put $f_{0}=f$ and assume that $f_{0}, \ldots, f_{n-1}$ have been defined and satisfy the above conditions. Let $W_{1}, \ldots, W_{v}$ be all the following equivariant subpolyhedra of $M$

(i) $V_{i}, i=1, \ldots, r$.

(ii) $\bigcap_{j=1}^{d} f_{n-1}\left(B_{j}\right)$, any $d$ equivariant simplexes $B_{1}, \ldots, B_{d}$ of $P_{n-1}-Q$ (d not fixed).

Let us denote $L=\operatorname{link}\left(A_{n} ; P^{*}\right)$, and $R_{0}=f_{n-1}\left(\dot{A}_{n} \cdot L\right)$, and $R=R_{0} \cup f_{n-1}\left(A_{n}\right)$. Then we have $R \cap \partial M \subset R_{0}$. Let $\varepsilon_{1}=\left(1 / 2^{n+1}\right) \min \left\{\varepsilon(x) \mid x \in A_{n} \cdot L\right\}$. By Proposition 3.8 there exists an equivariant ambient p.l. $\varepsilon_{1}$-isotopy $\Psi$ of $M \operatorname{rel}\left(\partial M \cup R_{0}\right)$ such that $\Psi_{1}\left(R-R_{0}\right)$ is in equivariant general position with respect to each $W_{1}, \ldots, W_{v}$. Now define $F^{(n)}: P \times I \rightarrow M$ by

$$
\begin{aligned}
F^{n}(x, t) & =\Psi\left(f_{n-1}(x), t\right)=\Psi_{t} f_{n-1}(x) \text { for } x \in A_{n} \cdot L \text { and } t \in I, \\
F^{(n)}(x, t) & =f_{n-1}(x) \text { for } x \in \overline{P-A_{n} \cdot L} \text { and } t \in I,
\end{aligned}
$$

and let $f_{n}(x)=F^{(n)}(x, 1)$. Thus $f_{n}(x)=\Psi_{1} f_{n-1}(x)$ if $x \in A_{n} \cdot L$ and $f_{n}(x)=f_{n-1}(x)$ if $x \in \overline{P-A_{n} \cdot L}$. Hence it follows that $f_{n}$ is equivariantly nondegenerate. Since $P_{n-1} \cap\left(A_{n} \cdot L\right) \subset \dot{A}_{n} \cdot L$ it follows that $F^{(n)}$ is an isovariant $\varepsilon / 2^{n+1}$-homotopy rel $P_{n-1}$ (in fact $F^{(n)}$ is also p.l. and proper). Also $f_{n}(P-Q) \subset$ int $M$.

Let us now show that $f_{n}$ satisfies condition 3 . First, using the fact that $f_{n-1}$ imbeds the equivariant simplexes of $P^{*}$, it is easily seen that $f_{n-1}\left(\AA_{n}\right)=R-R_{0}$. Thus we have $f_{n}\left(\AA_{n}\right)=\Psi_{1}\left(R-R_{0}\right)$. Since $\Psi_{1}\left(R-R_{0}\right)$ is in equivariant general position with respect to each $W_{j}$ we get

$$
\operatorname{dim}\left[\left(f_{n}\left(\check{A}_{n}\right) \cap W_{j}\right)_{H}\right]_{\beta} \leqslant \operatorname{dim}\left[\left(f_{n}\left(\AA_{n}\right)\right)_{H}\right]+\operatorname{dim}\left[\left(W_{j}\right)_{H}\right]_{\beta}-\operatorname{dim} M_{\beta}^{H},
$$

for any subgroup $H$ of $G$ and any component $M_{\beta}^{H}$ of $M^{H}$, and $j=1, \ldots, v$. Now let $s_{1}, \ldots, s_{k}$ be $k$ (any $k \geqslant 1$ ) distinct simplexes of $P_{n}^{*}-Q$, such that $f_{n}\left(s_{i}\right) \subset$ $M_{\beta}^{H}, i=1, \ldots, k$. Since $f_{n}\left|P_{n-1}^{*}=f_{n-1}\right| P_{n-1}^{*}$ we need only consider the case when at least one of the simplexes belongs to $P_{n}^{*}-P_{n-1}^{*}$. Since $f_{n}$ imbeds equivariant simplexes we see that we can assume that exactly one, say $s_{k}$, of the simplexes belongs to $P_{n}^{*}-P_{n-1}^{*}$. Then $f_{n}\left(\dot{s}_{k}\right) \subset\left[\left(f_{n}\left(\dot{A}_{n}\right)\right)_{H}\right]_{\beta}$, and denoting

$$
W=\bigcap_{i=1}^{k-1} f_{n-1}\left(G s_{i}\right) \cap V_{j}
$$

we get

$$
\operatorname{dim}\left(\left(\bigcap_{i=1}^{k} f_{n}\left(\stackrel{\bullet}{s}_{i}\right)\right) \cap V_{j}\right)=\operatorname{dim}\left(f_{n}\left(\stackrel{\circ}{s}_{k}\right) \cap W\right) \leqslant \operatorname{dim} s_{k}+\operatorname{dim}\left[W_{H}\right]_{\beta}-\operatorname{dim} M_{\beta}^{H}
$$

Since $f_{n-1} \mid: P_{n-1}^{*}-Q \rightarrow M$ is in equivariant strong general position with respect to each $V_{j}$, we have

$$
\operatorname{dim}\left[W_{H}\right]_{\beta} \leqslant \sum_{i=1}^{k-1} \operatorname{dim} s_{i}+\operatorname{dim}\left[\left(V_{j}\right)_{H}\right]_{\beta}-(k-1) \cdot \operatorname{dim} M_{\beta}^{H},
$$


and hence

$$
\operatorname{dim}\left(\left(\bigcap_{i=1}^{k} f_{n}\left(\dot{s}_{i}\right) \cap V_{j}\right)\right) \leqslant \sum_{i=1}^{k} \operatorname{dim} s_{i}+\operatorname{dim}\left[\left(V_{j}\right)_{H}\right]_{\beta}-k \operatorname{dim} M_{\beta}^{H} .
$$

This shows that $f_{n}$ satisfies condition 3 , and completes the inductive step.

Since $f_{n}$ equals $f_{n-1}$ outside $\left(A_{n} \cdot L\right)^{0}$ it follows that $h=\lim _{n \rightarrow \infty} f_{n}: P^{*} \rightarrow M$ is a well-defined equivariantly nondegenerate proper p.l. $G$-map such that $h \mid: P^{*}-Q$ $\rightarrow M$ is in equivariant strong general position with respect to $V_{j}, j=1, \ldots, r$. Moreover $h(P-Q) \subset$ int $M$.

The map $F_{0}: P \times I \rightarrow M$ defined by

$$
\begin{gathered}
F_{0}(x, t)=F^{(n)}\left(x, \frac{t-(1-1 / n)}{1 / n-1 /(n+1)}\right) \text { for } 1-1 / n \leqslant t<1-1 /(n+1), n \geqslant 1, \\
F_{0}(x, 1)=h(x)
\end{gathered}
$$

is a well-defined continuous isovariant homotopy from $f$ to $h$. Since $F_{0}$ is an $\varepsilon / 2$-homotopy from $f$ to $h$ it follows that $F_{0}$ is proper. Moreover $F_{0} \mid Q \times I=(f \mid Q)$ $\times$ id and hence $F_{0} \mid Q \times I$ is p.l. Thus by Corollary 3.4 there exists an isovariant proper p.l. $\varepsilon$-homotopy rel $Q$ from $f$ to $h$, such that $F\left|Q \times I=F_{0}\right| Q \times I$ and $F$ is an $\varepsilon / 2$-approximation to $F_{0}$. Then $F$ is an isovariant proper p.l. $\varepsilon$-homotopy rel $Q$ from $f$ to $h$. (In fact one can also easily show that already $F_{0}$ is p.l.)

THEOREM 3.10. Let $P$ be an equivariant polyhedron, $Q$ an equivariant subpolyhedron of $P$ and $M$ an equivariant p.l. manifold and $V_{1}, \ldots, V_{r}$ equivariant subpolyhedra of $M$, and let $\varepsilon: P \rightarrow \mathbf{R}_{+}$be any invariant positive function. Let $f: P \rightarrow M$ be $a$ proper $G$-map such that $f \mid: Q \rightarrow M$ is p.l. and equivariantly nondegenerate. Either assume that

$$
\operatorname{dim}\left(P_{\alpha}^{H}-\left(P^{>H} \cup Q\right)\right) \leqslant \operatorname{dim} M_{f(\alpha)}^{H}-\operatorname{dim} M_{f(\alpha)}^{>H}-1
$$

for every subgroup $H$ of $G$ and any component $P_{\alpha}^{H}$ of $P^{H}$, or assume that

$$
f \text { is isovariant and } \operatorname{dim}\left(P_{\alpha}^{H}-(P>H \cup Q)\right)<\operatorname{dim} M_{f(\alpha)}^{H}
$$

for every subgroup $H$ of $G$ and any component $P_{\alpha}^{H}$ of $P^{H}$. Then there exist an equivariant triangulation $P^{*}$ of $P$, inducing $Q^{*}$, and an equivariant proper $\varepsilon$-homotopy rel $|Q|$ from $f$ to an equivariantly nondegenerate proper p.l. $G$-map $h: P^{*} \rightarrow M$ such that $h \mid: P^{*}-Q^{*} \rightarrow M$ is in equivariant strong general position with respect to each $V_{i}, i=1, \ldots, r$. Moreover $h(P-Q) \subset$ int $M$.

Proof. This follows under assumption (1) from Theorems 3.1, 3.5 and Proposition 3.9, and under assumption (2) from Theorems 3.3, 3.7 and Proposition 3.9.

If in Theorem 3.10 we, to begin with, are given an equivariant simplicial complex $P^{\prime}$, then the equivariant simplicial complex $P^{*}$ of the conclusion can be chosen to be an equivariant subdivision of $P^{\prime}$. This is seen immediately from the proof of Theorem 3.10.

It should be observed that the condition to be in "equivariant strong general position" depends on the specific equivariant triangulation $P^{*}$ of $P$. If $h \mid: P^{*} \rightarrow Q^{*}$ $\rightarrow M$ is in equivariant strong general position (with respect to $V_{i}, i=1, \ldots, r$ ) and 
$P^{* *}$ is an equivariant subdivision of $P^{*}$ it does not follow that $h \mid: P^{* *}-Q^{* *} \rightarrow M$ is in equivariant strong general position (with respect to $V_{i}, i=1, \ldots, r$ ). But there are other forms of equivariant general position in terms of dimension estimates for the singular set

$$
S_{k}^{\prime}(h)=\left\{x \in P|| h^{-1} h(x) \mid \geqslant k\right\}
$$

that are of completely polyhedral nature and do not involve any particular equivariant triangulation. We leave it to the reader to formulate such results and deduce them as corollaries of Theorem 3.10.

As an important application of our equivariant general position result Theorem 3.10, we get the following two equivariant imbedding theorems. They follow from 3.10 be letting $V_{1}=M$ and $V_{2}=f(Q)=h(Q)$.

THEOREM 3.11. Let $f: P \rightarrow M$ be a proper $G$-map such that $f \mid Q$ is a p.l. $G$-imbedding. Let $\left(H_{1}\right), \ldots,\left(H_{v}\right)$ be the orbit types occurring in $P-Q$, and assume that

(i) $\operatorname{dim} P_{\alpha}^{H_{i}} \leqslant \operatorname{dim} M_{f(\alpha)}^{H_{i}}-\operatorname{dim} M_{f(\alpha)}^{>H_{i}}-1$,

(ii) $2 \operatorname{dim} P_{\alpha}^{H_{i}} \leqslant \operatorname{dim} M_{f(\alpha)}^{H_{i}}-1$,

for $i=1, \ldots, v$ and any component $P_{\alpha}^{H_{i}}$ of $P^{H_{i}}$. Then $f$ is equivariantly $\varepsilon$-homotopic rel $|Q|$ to a proper p.l. G-imbedding $h: P \rightarrow M$.

Proof. Since (i) holds it follows from Theorem 3.10 that there exist an equivariant triangulation $P^{*}$ of $P$, inducing $Q^{*}$, and an equivariant proper $\varepsilon$-homotopy rel $|Q|$ from $f$ to an equivariantly nondegenerate proper p.l. $G$-map $h: P^{*} \rightarrow M$ such that $h \mid: P^{*}-Q^{*} \rightarrow M$ is in equivariant strong general position with respect to $M$ and $f(Q)$. We claim that $h$ is a $G$-imbedding.

Since $h$ is proper it is enough to show that $h$ is injective. Let $x, y \in P$ such that $h(x)=h(y)$. We wish to prove that $x=y$. If $x, y \in Q$ this follows from the fact that $h|Q=f| Q$ is an imbedding. Next assume $x, y \in P-Q$. Since $h$ is isovariant it follows that $x$ and $y$ have the same isotropy subgroup, and furthermore we can assume that $G_{x}=G_{y}=H=H_{i}$, for some $i, 1 \leqslant i \leqslant v$. Let $M_{\beta}^{H}$ denote the component of $M^{H}$ for which $h(x)=h(y) \in M_{\beta}^{H}$. Since $f$ is $G$-homotopic to $h$ it follows that $f(x) \in M_{\beta}^{H}$ and $f(y) \in M_{\beta}^{H}$. Let $s_{1}$ and $s_{2}$ be the open simplexes of $P^{*}-Q^{*}$ such that $x \in \stackrel{\circ}{s}_{1}$ and $y \in \stackrel{\circ}{s}_{2}$, and let $P_{\alpha_{j}}^{H}$ denote the component of $P^{H}$ containing $\dot{s}_{j}, j=1,2$. Then $M_{f\left(\alpha_{1}\right)}^{H}=M_{\beta}^{H}=M_{f\left(\alpha_{2}\right)}^{H}$, and hence

$$
2 \operatorname{dim} P_{\alpha_{j}}^{H}-\operatorname{dim} M_{\beta}^{H} \leqslant-1, \quad j=1,2 .
$$

Now if $\stackrel{s}{1}_{1}$ and $\stackrel{s}{2}_{2}$ are distinct open simplexes it follows, since $h \mid P^{*}-Q^{*}$ is in equivariant strong general position, that

$$
\operatorname{dim}\left(h\left(\stackrel{\circ}{1}_{1}\right) \cap h\left(\stackrel{\circ}{s}_{2}\right)\right) \leqslant \operatorname{dim} s_{1}+\operatorname{dim} s_{2}-\operatorname{dim} M_{\beta}^{H}<-1,
$$

and hence $h\left(\stackrel{\circ}{1}_{1}\right) \cap h\left(\stackrel{\circ}{2}_{2}\right)=\varnothing$, which is in contradiction with the fact that $h(x)=$ $h(y) \in h\left(s_{1}\right) \cap h\left(\stackrel{s}{s}_{2}\right)$. Thus $\dot{s}_{1}=\stackrel{s}{2}_{2}$ and since $h \mid s_{1}$ is an imbedding it follows that $x=y$.

Finally assume that $x \in P-Q$ and $y \in Q$. Since $x$ belongs to $P-Q$ we may again assume that $G_{y}=G_{x}=H=H_{i}$, for some $i, 1 \leqslant i \leqslant v$. Let $P_{\alpha_{0}}^{H}$ be a 
component of $P^{H}$ such that $M_{f\left(\alpha_{0}\right)}^{H}=M_{\beta}^{H}$, and such that $\operatorname{dim} P_{\alpha^{\prime}}^{H} \leqslant \operatorname{dim} P_{\alpha_{0}}^{H}$ for every component $P_{\alpha^{\prime}}^{H}$ of $P^{H}$ with $M_{f\left(\alpha^{\prime}\right)}^{H}=M_{\beta}^{H}$. Then $2 \operatorname{dim} P_{\alpha_{0}}^{H}-\operatorname{dim} M_{\beta}^{H}<-1$. Let $s$ be the open simplex of $P^{*}-Q^{*}$ such that $x \in \stackrel{s}{ }$, and let $P_{\alpha}^{H}$ denote the component of $P^{H}$ containing $\stackrel{\circ}{ }$. Then we have $\operatorname{dim} P_{\alpha}^{H} \leqslant \operatorname{dim} P_{\alpha_{0}}^{H}$ and

$$
\operatorname{dim}\left[(h(Q))_{H}\right]_{\beta}=\operatorname{dim}\left[h(Q)_{H} \cap M_{\beta}^{H}\right] \leqslant \operatorname{dim} P_{\alpha_{0}}^{H} .
$$

Since $h \mid P^{*}-Q^{*}$ is in equivariant strong general position with respect to $h(Q)$ it now follows that

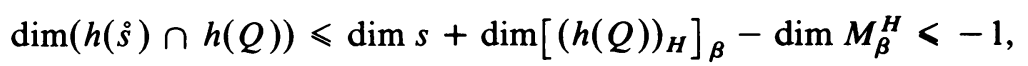

and hence that $h\left(s^{\circ}\right) \cap h(Q)=\varnothing$, a contradiction with $h(x)=h(y) \in h\left(s^{\circ}\right) \cap$ $h(Q)$. Thus $h(x)=h(y)$ is impossible if $x \in P-Q$ and $y \in Q$. This completes the proof of the fact that $h$ is injective and hence a $G$-imbedding.

THeOREM 3.12. Let $f: P \rightarrow M$ be an isovariant proper map such that $f \mid Q$ is a p.l. $G$-imbedding. Let $\left(H_{1}\right), \ldots,\left(H_{v}\right)$ be the orbit types occurring in $P-Q$, and assume that

$$
2 \operatorname{dim} P_{\alpha}^{H_{i}} \leqslant \operatorname{dim} M_{\left.f^{\prime} \alpha\right)}^{H_{i}}-1
$$

for $i=1, \ldots, v$ and any component $P_{\alpha}^{H_{i}}$ of $P^{H_{i}}$. Then $f$ is equivariantly $\varepsilon$-homotopic rel $|Q|$ to a proper p.l. G-imbedding $h: P \rightarrow M$.

Proof. Since $f$ is isovariant and we in particular have $\operatorname{dim} P_{\alpha}^{H_{i}}<\operatorname{dim} \boldsymbol{M}_{f(\alpha)}^{H_{i}}$, for $i=1, \ldots, v$ and any component $P_{\alpha}^{H_{i}}$ of $P^{H_{i}}$, it follows that Theorem 3.10 applies. The rest of the proof is the same as that for Theorem 3.11.

4. Equivariant imbeddings into euclidean representation space. In this section we consider the question of existence of $G$-imbeddings of equivariant simplicial complexes into euclidean representation space. Recall that all our simplicial complexes are assumed to be countable, locally finite and finite-dimensional. As soon as we know how to construct proper $G$-maps from equivariant simplicial complexes into euclidean representation spaces our general $G$-imbedding Theorem 3.11 can be applied and gives us the result stated in Theorem 4.2. The required result on the existence of proper $G$-maps is given by the following lemma.

LEMMA 4.1. Let $X$ be an equivariant simplicial complex and $Y$ an equivariant subcomplex of $X$. Let $\mathbf{R}^{n}(\rho)$ be a representation space for $G, f: Y \rightarrow \mathbf{R}^{n}(\rho)$ a proper $G$-map and assume that

$$
\operatorname{dim}(X-Y)^{H}+1 \leqslant \operatorname{dim}\left[\mathbf{R}^{n}(\rho)\right]^{H}
$$

for every subgroup $H$ of $G$. Then there exists a proper $G$-map $\bar{f}: X \rightarrow \mathbf{R}^{n}(\rho)$ such that $\bar{f} \mid Y=f$.

Proof. We may assume that $Y$ is full in $X$. Let $G x_{1}, G x_{2}, \ldots$ be the equivariant vertices of $X-Y$. Denote $G_{x_{j}}=H_{j}, j=1,2, \ldots$ Then, in any case, $\operatorname{dim}\left[\mathbf{R}^{n}(\rho)\right]^{H_{j}}$ $\geqslant 1$. Choose $v_{j} \in\left[\mathbf{R}^{n}(\rho)\right]^{H_{j}}$ such that $\left\|v_{j}\right\|=j, j=1,2, \ldots$ Then $\left\|g v_{j}\right\|=j$ for every $g \in G$ and $j=1,2, \ldots$ Denote $X_{m}=Y \cup X^{m}$, where $X^{m}$ denotes the $m$-skeleton of $X$, and let $k=\operatorname{dim} X$. If $G s_{1}$ is an equivariant simplex of $Y$ we 
denote

$$
d_{1}\left(G s_{1}\right)=\operatorname{dist}\left(f\left(G s_{1}\right), 0\right) .
$$

Let $G s_{2}$ be an equivariant simplex of $Y-X$ and let $G x_{j_{1}}, \ldots, G x_{j_{r}}$ be the equivariant vertices of $G s_{2}$ that belong to $X-Y$ (there is at least one since $Y$ is full in $X$ ). We then define

$$
d_{2}\left(G s_{2}\right)=\min \left\{j_{1}, \ldots, j_{r}\right\} .
$$

If now $G s$ is an arbitrary equivariant simplex of $X$ we define $d(G s)=\left\{\begin{array}{l}d_{1}(G s) \text { if } G s \subset Y, \\ \min \left(d_{1}(G s \cap Y), d_{2}(G s)\right) \text { if } G s \text { is a simplex of } X-Y \text { and } G s \cap Y \neq \varnothing, \\ d_{2}(G s) \text { if } G s \cap Y=\varnothing .\end{array}\right.$

We shall define $G$-maps $f_{m}: X_{m} \rightarrow \mathbf{R}^{n}(\rho), 0 \leqslant m \leqslant k$, such that

(i) $f_{m} \mid X_{m-1}=f_{m-1}$,

(ii) $f_{m}(G s) \subset \mathbf{R}^{n}(\rho)-\dot{D}^{n}(d(G s))$, for every equivariant simplex $G s$ of $X_{m}$.

Here $X_{-1}=Y$ and $f_{-1}=f$, and $\dot{D}^{n}(a)=\left\{x \in \mathbf{R}^{n}(\rho) \mid\|x\|<a\right\}$. Define $f_{0}$ : $X_{0} \rightarrow \mathbf{R}^{n}(\rho)$ by $f_{0} \mid X=f$ and $f_{0}\left(g x_{j}\right)=g v_{j}, j=1,2, \ldots$ Assume inductively that $f_{m-1}$ has been defined and satisfies (i) and (ii). Let $G s$ be an equivariant $m$-simplex of $X-Y$. Then $f_{m-1} \mid G \dot{s}$ is defined and since $d(G s) \leqslant d\left(G s^{\prime}\right)$ for every equivariant face $G s^{\prime}$ of $G s$ we have

$$
f_{m-1}(G \dot{s}) \subset \mathbf{R}^{n}(\rho)-\dot{D}^{n}(d(G s)) .
$$

Let $s=\left\langle y_{0}, \ldots, y_{m}\right\rangle$ and let $H$ denote the principal isotropy subgroup of $s$. Then $f_{m-1}(\dot{s}) \subset\left[\mathbf{R}^{n}(\rho)\right]^{H}-\dot{D}^{n}(d(G s))$, and

$$
q=\operatorname{dim}\left[\mathbf{R}^{n}(\rho)\right]^{H} \geqslant \operatorname{dim}(X-Y)^{H}+1 \geqslant m+1 .
$$

Since $\left[\mathbf{R}^{n}(\rho)\right]^{H}-D^{n}(d(G s))$ has the homotopy type of $S^{q-1}$, or $\mathbf{R}^{q}$ in case $d(G s)=$ 0 , and $m \leqslant q-1$ it follows that $f_{m-1} \mid \dot{s}$ can be extended to a map $f_{m}^{\prime}: s \rightarrow\left[\mathbf{R}^{n}(\rho)\right]^{H}$ - $D^{n}(d(G s))$. Then

$$
f_{m}: G s \rightarrow \mathbf{R}^{n}(\rho)-\check{D}^{n}(d(G s))
$$

defined by $f_{m}(g y)=g f_{m}^{\prime}(y)$, where $y \in s, g \in G$, is a well-defined $G$-map which extends $f_{m-1} \mid G \dot{s}$. Applying the above to every equivariant $m$-simplex of $X-Y$ we get a $G$-map $f_{m}: X_{m} \rightarrow \mathbf{R}^{n}(\rho)$ which satisfies conditions (i) and (ii). Let $\bar{f}=f_{k}$ : $X \rightarrow \mathbf{R}^{n}(\rho)$.

We claim that $\bar{f}$ is proper. Let $C \subset \mathbf{R}^{n}(\rho)$ be compact. Let $a>0$ be such that $C \subset D^{n}(a)$. Since $\bar{f} \mid Y=f$ is proper there are only finitely many equivariant simplexes, say $G s_{1}, \ldots, G s_{t}$, of $Y$ such that

$$
\bar{f}\left(G s_{i}\right) \cap D^{n}(a) \neq \varnothing .
$$

Let $j_{0}>a, j_{0} \in \mathbf{N}$. It now follows that only the equivariant simplexes $G s$ of $X$ that either have as a face one of the simplexes $G s_{1}, \ldots, G s_{t}$ or have as a vertex one of the equivariant simplexes $G x_{1}, \ldots, G x_{j_{0}}$ are such that possibly $\bar{f}(G s) \cap D^{n}(a) \neq \varnothing$. Thus $\bar{f}^{-1}\left(D^{n}(a)\right)$ is compact and hence also $\bar{f}^{-1}(C)$. 
TheOREM 4.2. Let $X$ be a simplicial $G$-complex and $X_{0}$ a $G$-subcomplex of $X$, and let $\left(H_{1}\right), \ldots,\left(H_{v}\right)$ be the orbit types occurring in $X-X_{0}$. Let $\mathbf{R}^{r}(\rho)$ be a representation space for $G$ such that there exists a proper p.l. G-imbedding $h_{0}: X_{0} \rightarrow \mathbf{R}^{r}(\rho)$ and assume that

(i) $\operatorname{dim} X^{H_{i}} \leqslant \operatorname{dim}\left[\mathbf{R}^{r}(\rho)\right]^{H_{i}}-\operatorname{dim}\left[\mathbf{R}^{r}(\rho)\right]^{>H_{i}}-1$,

(ii) $2 \operatorname{dim} X^{H_{i}} \leqslant \operatorname{dim}\left[\mathbf{R}^{r}(\rho)\right]^{H_{i}}-1$

for $i=1, \ldots, v$. Then $h_{0}$ can be extended to a proper p.l. G-imbedding $h: X \rightarrow$ $\mathbf{R}^{r}(\rho)$.

Proof. It follows from Lemma 4.1 that there exists a proper $G$-map $f: X \rightarrow \mathbf{R}^{r}(\rho)$ such that $f \mid X_{0}=f_{0}$. The result now follows from Theorem 3.11.

Let $G=Z_{m}=\left\{e, T, \ldots, T^{m-1}\right\}$, where $m \geqslant 2$. We shall use the following notation. If $p^{s} \mid m$, where $p$ is an odd prime and $s>1$ or $p=2$ and $s>2$, we denote by $\mathbf{R}^{2}\left(1 / p^{s}\right)$ the euclidean plane $\mathbf{R}^{2}=\mathbf{C}$ together with $Z_{m}$-action given by $T z=\exp \left(2 \pi i / p^{s}\right) z$, where $\exp (y)=e^{y}$. For $m$ even we denote by $\mathbf{R}( \pm)$ the real line with $Z_{m}$-action given by $T x=-x$. Furthermore, for any integer $k \geqslant 0$, we denote

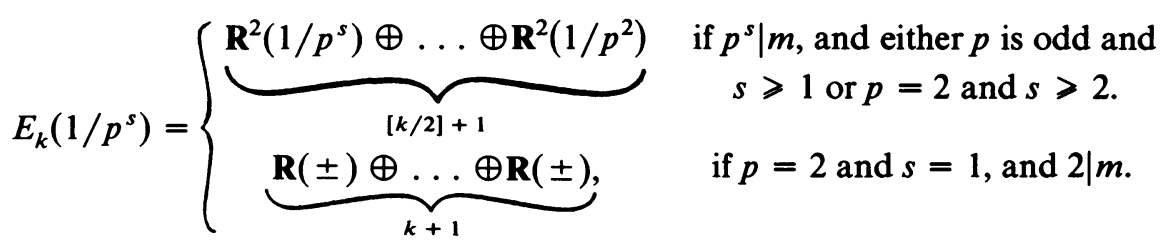

The dimensions of these spaces are given by

$$
\operatorname{dim} E_{k}\left(1 / p^{s}\right)= \begin{cases}k+2 & \text { if } k \text { is even and } p \text { is odd or } p=2 \text { and } s>2, \\ k+1 & \text { if } k \text { is odd or if } p=2 \text { and } s=1\end{cases}
$$

Our Theorem 4.2 now gives us

Corollary 4.3. Let $m=p_{1}^{a_{i}} \cdots p_{n}^{a_{n}}$, where $p_{1}, \ldots, p_{n}$ are distinct primes, and $k \geqslant 0$ be integers. Define $\mathbf{R}^{r(k, m)}(\rho)$ to be the euclidean representation space

$$
\begin{aligned}
\mathbf{R}^{r(k, m)}(\rho)= & \mathbf{R}^{2 k+1} \oplus E_{k}\left(1 / p_{1}\right) \oplus \ldots \oplus E_{k}\left(1 / p_{1}^{a_{1}}\right) \\
& \oplus \ldots \oplus E_{k}\left(1 / p_{n}\right) \oplus \ldots \oplus E_{k}\left(1 / p_{n}^{a_{n}}\right) .
\end{aligned}
$$

Then any $k$-dimensional simplicial $Z_{m}$-complex $X$ admits a proper p.l. $Z_{m}$-imbedding into $\mathbf{R}^{r(k, m)}(\rho)$. The dimension $r(k, m)$ is given by

$$
r(k, m)= \begin{cases}2 k+1+\left(a_{1}+\cdots+a_{n}\right)(k+2) & \text { if } k \text { is even and } m \text { is odd, } \\ 2 k+\left(a_{1}+\cdots+a_{n}\right)(k+2) & \text { if } k \text { is even and } m \text { is even, } \\ 2 k+1+\left(a_{1}+\cdots+a_{n}\right)(k+1) & \text { if } k \text { is odd }\end{cases}
$$

Proof. It is straightforward to see that $\mathbf{R}^{r(k, m)}(\rho)$ satisfies the two conditions in Theorem 4.2 for every subgroup $H$ of $Z_{m}$.

Let $m=p$, a prime. In Copeland-de Groot [3] it is proved that every $k$-dimensional, separable, metrizable $Z_{p}$-space admits a $Z_{p}$-imbedding into $\mathbf{R}^{r(k, p)}$. The 
dimension $r(k, p)$ is given by

$$
r(k, p)= \begin{cases}3 k+3 & \text { if } k \text { is even and } p \neq 2 \\ 3 k+2 & \text { if } k \text { is odd or } p=2\end{cases}
$$

Thus in the special case of actions of cyclic groups of prime order our result only contributes the addition to the result by Copeland and de Groot that in the case of simplicial $Z_{p}$-complexes the $Z_{p}$-imbedding can moreover be chosen to be p.l. (and proper). Furthermore Copeland and de Groot show (see $\$ 4$ in [3]) that their result is best possible and since their examples are (finite) simplicial $Z_{p}$-complexes it also follows that our result Corollary 4.3 is best possible in the case when $m=p$, a prime. We shall show in Proposition 4.4 that our result Corollary 4.3 in fact is best possible for every finite cyclic group $Z_{m}$.

In Kister-Mann [9] $G$-imbeddings into euclidean representation space of finite dimensional separable metrizable $G$-spaces, where $G$ is a compact abelian Lie group, are considered. For $G=Z_{m}$, where $m=p_{1}^{a_{1}} \ldots p_{n}^{a_{n}}$, their result proves that any $k$-dimensional separable metrizable $Z_{m}$-space can be $Z_{m}$-imbedded into a euclidean representation space of dimension $s(k, m)$, where

$$
s(k, m)=\left\{\begin{array}{l}
2 k+1+\left(\left(a_{1}+1\right)\left(a_{2}+1\right) \ldots\left(a_{n}+1\right)-1\right)(k+2) \\
3 k+2 \quad \text { if } k \text { is even and } m=2, \\
2 k+1+\left(\left(a_{1}+1\right)\left(a_{2}+1\right) \ldots\left(a_{n}+1\right)-1\right)(k+1) \\
\text { if } k \text { is odd }
\end{array}\right.
$$

Thus in case $m=p$, a prime, the dimension given by Kister-Mann is the same as the one given by Copeland-de Groot and by our result. If $m$ is a prime power, $m=p^{a}$ where $a \geqslant 2$, the dimension given by the Kister-Mann result equals the one given in our Corollary 4.3 if $p \neq 2$ or if $k$ is odd, and in case $k$ is even and $m=2^{a}, a \geqslant 2$, the Kister-Mann dimension exceeds ours by one. Thus in the case of actions of cyclic groups of prime power order our dimension result is only a slight improvement of the Kister-Mann result. But for example in the case when $m$ is the product of distinct primes, $m=p_{1} \ldots p_{n}$, where $n \geqslant 2$, the dimension given by our Corollary 4.3 is by far much lower than the dimension given by the Kister-Mann result. In this case our result gives the dimension

$$
r\left(k, p_{1} \ldots p_{n}\right)= \begin{cases}(n+2) k+2 n+1 & \text { if } k \text { is even and } m=p_{1} \ldots p_{n} \text { is odd } \\ (n+2) k+2 n & \text { if } k \text { is even and } m \text { is even, } \\ (n+2) k+n+1 & \text { if } k \text { is odd }\end{cases}
$$

whereas the dimension obtained by the Kister-Mann result is

$$
s\left(k, p_{1} \ldots p_{n}\right)= \begin{cases}\left(2^{n}+1\right) k+2^{n+1}-1 & \text { if } k \text { is even } \\ \left(2^{n}+1\right) k+2^{n} & \text { if } k \text { is odd }\end{cases}
$$

Let us now show that our result Corollary 4.3 is in fact best possible. 
Proposition 4.4. Let $m \geqslant 1$ and $k \geqslant 0$ be integers and let $r(k, m)$ be as in Corollary 4.3. Then there exists a $k$-dimensional finite simplicial $Z_{m}$-complex which cannot be $Z_{m}$-imbedded (not even topologically) into any euclidean representation space of dimension $r(k, m)-1$.

Proof. Let $p$ be a prime dividing $m$ and let $p^{a}$ be the highest power of $p$ that divides $m$. Let $1 \leqslant s \leqslant a$. We define a $k$-dimensional simplicial $Z_{p^{s} \text {-complex }}$

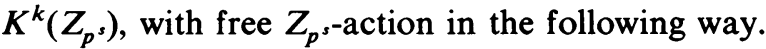

(1) If $k$ is odd we let

$$
K^{k}\left(Z_{p^{s}}\right)=S^{k}
$$

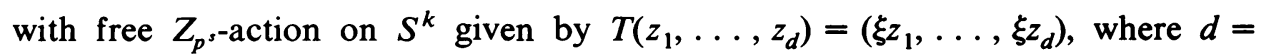
$(k+1) / 2$ and $\left(z_{1}, \ldots, z_{d}\right) \in S\left(C^{d}\right)=S^{2 d-1}$ and $\xi=\exp \left(2 \pi i / p^{s}\right)$.

(2) If $k$ is even we let

$$
K^{k}\left(Z_{p^{s}}\right)=\left(D^{k} \times Z_{p^{s}}\right) / \sim
$$

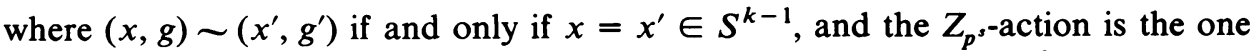

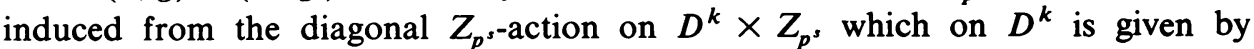
$T\left(z_{1}, \ldots, z_{d}\right)=\left(\xi z_{1}, \ldots, \xi z_{d}\right)$, where $d=k / 2$ and $\left(z_{1}, \ldots, z_{d}\right) \in D\left(C^{d}\right)=D^{2 d}$ and $\xi=\exp \left(2 i / p^{s}\right)$, and on $Z_{p^{s}}$ is given by multiplication on the left. Observe that $K^{k}\left(Z_{2}\right)$ is a $k$-dimensional sphere with the antipodal action also in the case when $k$ is even.

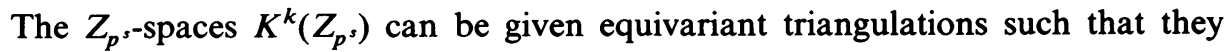

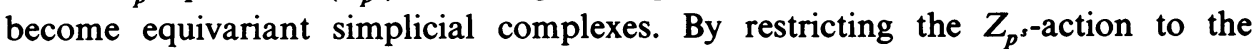
subgroup $Z_{p} \hookrightarrow Z_{p^{s}}$ each $K^{k}\left(Z_{p^{s}}\right)$ becomes a $Z_{p}$-space. We then have the $Z_{p}$-imbedding

$$
\iota: K^{k}\left(Z_{p}\right) \hookrightarrow K^{k}\left(Z_{p^{s}}\right)
$$

which for $k$ odd is the identity map and for $k$ even is induced from the $Z_{p}$-imbedding $D^{k} \times Z_{p} \hookrightarrow D^{k} \times Z_{p^{s}}$.

We make each $K^{k}\left(Z_{p^{s}}\right)$ into a $Z_{m}$-space by changing the $Z_{p}$-action to a $Z_{m}$-action through the natural projection $Z_{m} \rightarrow Z_{p}$. Then every point in the $Z_{m}$-space $K^{k}\left(Z_{p^{s}}\right)$ has isotropy subgroup equal to $p^{s} Z_{m} \subset Z_{m}$.

Now assume that we are given a $Z_{m}$-imbedding

$$
h: K^{k}\left(Z_{p^{s}}\right) \rightarrow V,
$$

where $V$ is a euclidean representation space, which we may assume to be an orthogonal representation space for $Z_{m}$. Let us denote $H_{1}=p^{s} Z_{m}, H_{0}=p^{s-1} Z_{m}$, $V_{1}=V^{H_{1}}$ and $V_{0}=V^{H_{0}}$. Then $\operatorname{Im}(h) \subset V_{1}$ and $\operatorname{Im}(h) \cap V_{0}=\varnothing$. Thus

$$
\bar{h}=\pi \circ h: K^{k}\left(Z_{p^{s}}\right) \rightarrow V_{1} \rightarrow V_{1} / V_{0}=W
$$

is a $Z_{m}$-map with $\operatorname{Im}(\bar{h}) \cap\{0\}=\varnothing$. Hence $\hat{h}$ defined by $\hat{h}(y)=\bar{h}(y) /\|\bar{h}(y)\|$ is a $Z_{m}$-map

$$
\hat{h}: K^{k}\left(Z_{p^{s}}\right) \rightarrow S(W)=S^{w-1},
$$

where $w=\operatorname{dim} W=\operatorname{dim} V_{1}-\operatorname{dim} V_{0}$. 
Since every point in the $Z_{m}$-space $V_{1}$ is fixed under $p^{s} Z_{m}$ it follows that

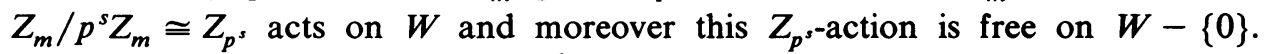
Likewise $Z_{m} / p^{s} Z_{m} \cong Z_{p}$ s acts on $K^{k}\left(Z_{p^{s}}\right)$ and this action is just the original given

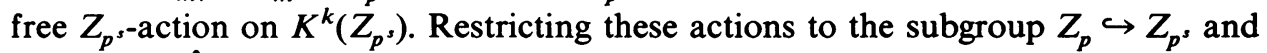
composing $\hat{h}$ with $\iota$ we get the $Z_{p}$-map

$$
\hat{h} \circ \iota: K^{k}\left(Z_{p}\right) \rightarrow S(W)=S^{w-1} .
$$

If $p=2$ this implies (see p. 91 in [3]) that $k \leqslant w-1$. If $p=2$ and $s \geqslant 2$ we moreover have that $w$ must be even since $Z_{2^{\text {s }}}$ acts freely on $W-\{0\}$, and hence in case $k$ is even we have $k \leqslant w-2$. Thus for $p=2$ we have

$$
\begin{array}{ll}
k+1 \leqslant w & \text { if } k \text { is odd or if } s=1 \\
k+2 \leqslant w & \text { if } k \text { is even and } s \geqslant 2 .
\end{array}
$$

For $p \neq 2$ the argument is Copeland-de Groot [3] shows that

$$
\begin{array}{ll}
k+1 \leqslant w & \text { if } k \text { is odd, } \\
k+2 \leqslant w & \text { if } k \text { is even. }
\end{array}
$$

Now let $m=p_{1}^{a_{1}} \cdots p_{n}^{a_{n}}$, where $p_{1}, \ldots, p_{n}$ are distinct primes. Let $A^{k}$ be the $k$-skeleton of the $(2 k+2)$-simplex. Then by Flores [5] $A^{k}$ does not imbed into euclidean space of dimension less than $2 k+1$. We define a $k$-dimensional finite simplicial $Z_{m}$-complex $X$ by

$$
X=A^{k} \dot{\cup} K^{k}\left(Z_{p_{1}}\right) \dot{\cup} \ldots \dot{\cup} K^{k}\left(Z_{p_{1}^{a_{1}}}\right) \dot{\cup} \ldots \dot{\cup} K^{k}\left(Z_{p_{n}}\right) \dot{\cup} \ldots \dot{\cup} K^{k}\left(Z_{p_{n}^{q_{n}}}\right) \text {, }
$$

where $\dot{U}$ denotes the disjoint union. Assume we are given a $Z_{m}$-imbedding $f$ : $X \rightarrow V$. If $H=p_{i}^{a_{i}} Z_{m}$ and $H^{\prime}=p_{j}^{a_{j}} Z_{m}$, where $i \neq j$, we have $V^{H} \cap V^{H^{\prime}}=V^{Z_{m}}$. Hence it now follows that we must have $\operatorname{dim} V \geqslant r(k, m)$.

Corollary 4.5. Let $X$ be a $k$-dimensional simplicial $Z_{m}$-complex with semi-free $Z_{m}$-action and assume that $X^{Z_{m}}$ has a proper p.l. imbedding $h_{0}$ into $\mathbf{R}^{d}$. Then $X$ has a proper p.l. $Z_{m}$-imbedding, extending $h_{0}$, into a euclidean representation space $\mathbf{R}^{e}(\rho)$, where

$$
e= \begin{cases}\max (k-1, d)+k+2 & \text { if } k \text { is even and } m \neq 2 \\ \max (k, d)+k+1 & \text { if } k \text { is odd or } m=2\end{cases}
$$

Proof. The representation $R^{e}(\rho)$ is the direct sum of $\mathbf{R}^{\max (k-1, d)}\left[\mathbf{R}^{\max (k, d)}\right]$ and a representation space $\mathbf{R}^{k+2}\left(\rho^{\prime}\right)\left[\mathbf{R}^{k+1}\left(\rho^{\prime}\right)\right]$ with $Z_{m}$-action which is free outside the origin. The claim now follows from Theorem 4.2 .

For compact, finite-dimensional metric spaces the topological version of Corollary 4.5 has been proved by $R$. Allen (see [1, Theorems 1.1 and 1.2]). In particular Corollary 4.5 shows that all $k$-dimensional semi-free simplicial $Z_{m^{-}}$ complexes $X$ with $\operatorname{dim} X^{Z_{m}}<k / 2$ have a $Z_{m}$-imbedding into a euclidean representation space of dimension $2 k+1$.

In the case of semi-free actions of noncyclic finite abelian groups we obtain the following. 
Corollary 4.6. Let $G=R_{1} \oplus \ldots \oplus R_{r} \oplus S_{1} \oplus \ldots \oplus S_{s}$, where $r+s \geqslant 2$ and the $R$ 's are finite cyclic groups of order $\neq 2$ and the $S$ 's are cyclic of order 2. Let $X$ be a $k$-dimensional simplicial $G$-complex with semi-free $G$-action, and assume that $X^{G}$ has a proper p.l. imbedding $h_{0}$ into $\mathbf{R}^{d}$. Then $X$ has a proper p.l. G-imbedding, extending $h_{0}$, into a euclidean representation space $\mathbf{R}^{e}(\rho)$, where

$$
e= \begin{cases}d+r(k+2)+s(k+1) & \text { if } k \text { is even, } \\ d+(r+s)(k+1) & \text { if } k \text { is odd } .\end{cases}
$$

Proof. For $k$ odd any finite cyclic group has a linear action, which is free outside the origin, on $(k+1)$-dimensional euclidean space. The direct sum of $r+s$ copies of such spaces plus a copy of $\mathbf{R}^{d}$ gives the desired representation space $\mathbf{R}^{e}(\rho)$ for $G$, and an application of Theorem 4.2 proves the claim. The proof is completely analogous in the case when $k$ is even.

Taking $d=2 k+1$ we see that any semi-free $k$-dimensional simplicial $G$-complex has a proper p.l. $G$-imbedding into a $(2 k+1+r(k+2)+s(k+1))$ - or $(2 k+1+(r+s)(k+1))$-dimensional, for $k$ even and odd respectively, euclidean representation space. In this situation the dimensions are the same as the ones given by Kister-Mann [9, Theorem 1] in the topological case. In their proof the factor $\mathbf{R}^{2 k+1}$ comes from an imbedding of the orbit space $X / G$, whereas in our case it comes from an imbedding of the fixed point set $X^{G}$, and hence their proof does not establish a topological version of Corollary 4.6.

We now turn to the case of arbitrary actions of finite abelian groups, and compared with corresponding result by Kister-Mann (see [9, Theorem 2]) we obtain an improvement of the required dimension of the euclidean representation space. Let $G$ be a finite abelian group. We say that $H_{1}, \ldots, H_{v}$ are generating subgroups of $G$ if any subgroup $H$ of $G$, where $H \neq G$, is an intersection of some of the $H_{i}$ 's.

Corollary 4.7. Let $G$ be a finite abelian group as in Corollary 4.6 and let $v=v(G)$ be the minimal number of generating subgroups of $G$. Then there exists a euclidean representation space $\mathbf{R}^{w}(\rho)$, where

$$
w= \begin{cases}2 k+1+v[r(k+2)+s(k+1)] & \text { if } k \text { is even } \\ 2 k+1+v(r+s)(k+1) & \text { if } k \text { is odd }\end{cases}
$$

such that any $k$-dimensional simplicial G-complex admits a proper p.l. G-imbedding into $\mathbf{R}^{w}(\rho)$.

Proof. Assume that $k$ is odd. Let $H \neq G$ be a subgroup of $G$. Then, as described in the proof of Corollary 4.6, the group $G / H$ has a representation space $V$ of dimension $(r+s)(k+1)$ such that $\operatorname{dim} V^{\{e\}}-\operatorname{dim} V^{>\{e\}}=k+1$. Considering $V$ as a representation space for $G$ we then have $\operatorname{dim} V^{H}-\operatorname{dim} V^{>H}=k+1$. Let $H_{1}, \ldots, H_{v}$ be generating subgroups of $G$. For each $H_{i}, i=1, \ldots, v$, we let $V_{i}$ be a representation space of the type described above. Then, letting $\mathbf{R}^{\omega}(\rho)=\mathbf{R}^{2 k+1} \oplus$ $V_{1} \oplus \ldots \oplus V_{v}$, the result follows by Theorem 4.2. The proof in the case when $k$ is even is completely analogous. 
In the corresponding result by Kister-Mann (see [9, Theorem 2]) the factor $v$ is replaced by the total number $t_{0}$ of subgroups $H$ of $G$, with $H \neq G$. (Corollary 4.7 is also valid as stated in case $r+s=1$, i.e., $r=1$ and $s=0$ or $r=0$ and $s=1$. But in this case $G$ is cyclic and the result has already been covered by Corollary 4.3, which in fact is a little sharper in the case when $G$ is cyclic of even order $m$, where $m \neq 2$, and $k$ is even.)

In order to give an application of Theorem 4.2 for a nonabelian group we present the following example.

EXAmPLE. Let $G=S_{3}$ the symmetric group on three letters. Let $x, y \in G$ with $x^{2}=1, y^{3}=1$ and $x y=y^{2} x$. There are altogether six subgroups of $G$ and they are: $G, K=\left\{1, y, y^{2}\right\}, H=\{1, x\}$ and two conjugates of $H$ and $\{1\}$. Let $\mathbf{R}^{3}(\sigma)$ denote $\mathbf{R}^{3}$ together with the $G$-action which permutes the coordinate axes. Then we have $\operatorname{dim}\left[\mathbf{R}^{3}(\sigma)\right]^{G}=1$ and $\operatorname{dim}\left[\mathbf{R}^{3}(\sigma)\right]^{H}-\operatorname{dim}\left[\mathbf{R}^{3}(\rho)\right]^{>H}=1$ and $\operatorname{dim}\left[\mathbf{R}^{3}(\sigma)\right]^{\{1\}}-$ $\operatorname{dim}\left[\mathbf{R}^{3}(\sigma)\right]^{>\{1\}}=1$. Let $\mathbf{R}( \pm)$ denote the euclidean line $\mathbf{R}$ together with $G$-action given by $x a=-a$ and $y a=a$, for every $a \in \mathbf{R}$. Then $\operatorname{dim}[\mathbf{R}( \pm)]^{K}-$ $\operatorname{dim}[\mathbf{R}( \pm)]^{>K}=1$. Hence Theorem 4.2 implies that

$$
\mathbf{R}^{5 k+4}(\rho)=\mathbf{R}^{k} \oplus\left[\mathbf{R}^{3}(\sigma)\right]^{k+1} \oplus[\mathbf{R}( \pm)]^{k+1}
$$

is such a representation space for $S_{3}$ that any $k$-dimensional simplicial $S_{3}$-complex has a proper p.l. $S_{3}$-imbedding into $\mathbf{R}^{5 k+4}(\rho)$.

Let us conclude by showing that the p.l. $G$-imbeddings of equivariant p.l. manifolds into euclidean representation space given by our $G$-imbedding result are in general equivariantly locally knotted although they are locally flat in the ordinary sense. This phenomenon can occur in arbitrarily high codimensions. Among other things this shows that the existence of p.l. and smooth $G$-imbeddings are questions of completely different nature.

EXAMPLE. Let $G=Z_{5}$ and let $\mathbf{R}^{2}\left(\rho_{1}\right)$ and $\mathbf{R}^{2}\left(\rho_{2}\right)$ denote the euclidean plane with $Z_{5}$-action given by $T z=\exp (2 \pi i / 5) z$ and $T z=\exp (4 \pi i / 5) z$, respectively. Let $M=D^{2}\left(\rho_{2}\right)$. By Theorem 4.2 there exists a p.l. $Z_{5}$-imbedding of $M$ into $\mathbf{R} \oplus \mathbf{R}^{2}\left(\rho_{1}\right)$ $\oplus \mathbf{R}^{2}\left(\rho_{1}\right)$ (and also into $\mathbf{R}^{2}\left(\rho_{1}\right) \oplus \mathbf{R}^{2}\left(\rho_{1}\right) \oplus \mathbf{R}^{2}\left(\rho_{1}\right)$ ). But any p.l. $Z_{5}$-imbedding $h$ : $M \rightarrow \mathbf{R}^{n} \oplus\left(\mathbf{R}^{2}\left(\rho_{1}\right)\right)^{k}=V$, where $n \geqslant 1$ and $k \geqslant 2$ (or $n \geqslant 0$ and $k \geqslant 3$ ), is equivariantly knotted at $h(0)$. That is, denoting $h(M)=N$, we have for any equivariant triangulation $V^{*}$ of $V$ inducing an equivariant triangulation $N^{*}$ of $N$ with $h(0)=v$ a vertex, the ball-pair $\left(\operatorname{St}\left(v, V^{*}\right), \operatorname{St}\left(v, N^{*}\right)\right)$ is not equivariantly p.l. homeomorphic with any standard ball-pair $\left(D^{m}(\tau), D^{2}\left(\tau^{\prime}\right)\right)$, where $m=n+2 k$. For if these ball-pairs were equivariantly p.l. homeomorphic it would follow by de Rham [11] that $\tau$ is isomorphic to

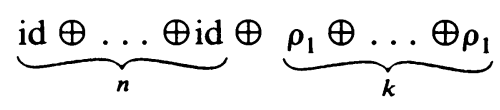

and that $\tau^{\prime}$ is isomorphic to $\rho_{2}$. Since $\tau$ contains $\tau^{\prime}$ as a direct summand we would have that

$$
\underbrace{\mathrm{id} \oplus \ldots \oplus \mathrm{id}}_{n} \oplus \underbrace{\rho_{1} \oplus \ldots \oplus \rho_{1}}_{k}
$$


contains $\rho_{2}$, a contradiction. In fact using Theorem I in Schultz [13], instead of the result by de $\operatorname{Rham}$, we see that $\left(\operatorname{St}\left(v, V^{*}\right), \operatorname{St}\left(v, N^{*}\right)\right)$ is even topologically equivariantly knotted, i.e. $\left(\operatorname{St}\left(v, V^{*}\right), \operatorname{St}\left(v, N^{*}\right)\right)$ is not $Z_{5}$-homeomorphic with any standard ball-pair $\left(D^{m}(\tau), D^{2}\left(\tau^{\prime}\right)\right)$. On the other hand, since $m-2>3$, we have by Zeeman [16] that $\left(\operatorname{St}\left(v, V^{*}\right), \operatorname{St}\left(v, N^{*}\right)\right)$ is p.l. homeomorphic with $\left(D^{m}, D^{2}\right)$.

To get an example with $M$ a compact equivariant p.l. manifold without boundary take $M=S^{2}\left(\rho_{2} \oplus\right.$ id). If we do not insist on $M$ being compact we may as well take $M=\mathbf{R}^{2}\left(\rho_{2}\right)$.

\section{REFERENCES}

1. R. J. Allen, Equivariant embeddings of $Z_{p}$-actions in euclidean space, Fund. Math. 103 (1979), 23-30.

2. G. E. Bredon, Introduction to compact transformation groups, Academic Press, New York and London, 1972.

3. A. H. Copeland, Jr. and J. de Groot, Linearization of a homeomorphism, Math. Ann. 144 (1961), 80-92.

4. A. L. Edmonds, Triangulation of piecewise linear group actions (unpublished note).

5. A. Flores, Über $n$-dimensionale Komplexe, die im $R_{2 n+1}$ absolut selbstverschlungen sind, Ergebnisse eines Math. Kolloq. 6 (1933/34), 4-7.

6. J. F. P. Hudson, Piecewise linear topology, Benjamin, New York, 1969.

7. S. Illman, Approximation of simplicial G-maps by equivariantly nondegenerate maps, in Transformation groups (Proc. Conf. Univ. of Newcastle upon Tyne, August 1976), C. Kosniowski (Ed.), London Math. Soc. Lecture Note Series, no. 26, Cambridge Univ. Press, Cambridge, 1977, pp. 279-283.

8. _ Smooth equivariant triangulations of $G$-manifolds for $G$ a finite group, Math. Ann. 233 (1978), 199-220.

9. J. M. Kister and L. N. Mann, Equivariant imbeddings of compact abelian Lie groups of transformations, Math. Ann. 148 (1962), 89-93.

10. G. D. Mostow, Equivariant embeddings in Euclidean space, Ann. of Math. (2) 65 (1957), $432-446$.

11. G. de Rham, Reidemeister's torsion invariant and rotations of $S^{n}$, in Differential Analysis, Tata Inst. and Oxford Univ. Press, London, 1964, pp. 27-36.

12. T. B. Rushing, Topological embeddings, Academic Press, New York and London, 1973.

13. R. Schultz, On the topological classification of linear representations, Topology 16 (1977), 263-269.

14. M. H. Schwartz, Théoreme d'approximation simpliciale lié à une stratification, Mathematica (Cluj) 13 (1971), 301-334.

15. A. G. Wasserman, Equivariant differential topology, Topology 8 (1969), 127-150.

16. E. C. Zeeman, Unknotting combinatorial balls, Ann. of Math. (2) 78 (1963), 501-526.

INSTITUT DES HaUtes Études ScIentifiQues, BuRES-SUR-YvetTE, France

Department of Mathematics, University of Helsinki, Hallituskatu 15, 00100 Helsinki 10 , FinLAND 